\title{
Response of Fish to Different Simulated Rates of Water Temperature Increase $(\mathrm{U})$
}

L. D. Wike and R. C. Tuckfield

Westinghouse Savannah River Company

Savannah River Site

Aiken, SC 29808

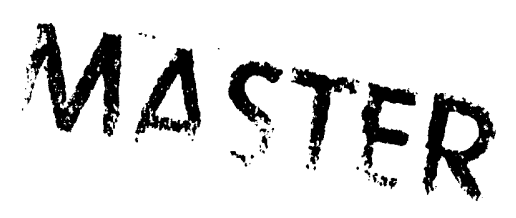

Prepared for the U. S. Department of Energy under contract No. DE-AC09-89SR18035 


\title{
Contents
}

\author{
Abstract 1 \\ Introduction 1 \\ Methods 2 \\ Laboratory Facility 2 \\ Simulation Apparatus 3 \\ Experimental Design 3 \\ Data Analysis 4 \\ Results 5 \\ System Performance 5 \\ Rate-Effect Experiment 5 \\ Acclimation-Effect Experiment 8 \\ Summary and Conclusions 8 \\ Options fo: Additional Studies 8
}

Acknowledgments 10

References 10

Appendix A 11

Appendix B 29

Appendix C 63 


\section{List of Figures}

Figure 1. General Layout of the Par Pond Fisheries Laboratory (735-7G) (page 2)

Figure 2. One of Four Troughs Used in the Thermal Simulation System (page 3)

Figure 3. One of 36 Individual Tanks Used in the Thermal Simulation System (page 3)

Figure 4. The Thermal Simulation System (page 4)

Figure 5. Examples of General and Tank-Specific Däta Sheets (page 4)

Figure 6. Survivorship Curves for Rate Experiment (page 7)

Figure 7. Survivorship Curves for Acclimation Experiment (page 7)

Figure 8. Scope for Activity (Kelsch and Neill 1990) (page 9) 


\title{
Response of Fish to Different Simulated Rates of Water Temperature Increase
}

\author{
L. D. Wike and R. C. Tuckfield \\ Westinghouse Savannah River Company \\ Savannah River Site \\ Aiken, SC 29808
}

\begin{abstract}
We initiated this study to define the limits of effluent-temperature rate increases during reactor restart, which will help minimize fish kills. We constructed an apparatus for exposing fish to various temperature-increase regimens and conducted two experiments based on information from system tests and scoping runs. In the rate experiment, we acclimated the fish to $20^{\circ} \mathrm{C}$, and then raised the temperature to $40^{\circ} \mathrm{C}$ at varying rates. Because scoping runs and literature suggested that acclimation temperature may affect temperature-related mortality, we conducted an acclimation experiment. We acclimated the fish to various temperatures, then raised the temperatures to $39-40^{\circ} \mathrm{C}$ at a rate of $2^{\circ} \mathrm{C}$ every 12 hours. Based on the analysis of the data, we recommend temperature-increase rates during reactor restart of $2.5^{\circ} \mathrm{C}$ every nine hours if ambient water temperatures are over $20^{\circ} \mathrm{C}$. If water temperatures are at or below $20^{\circ} \mathrm{C}$, we recommend temperature-increase rates of $2.5^{\circ} \mathrm{C}$ every 12 hours. No regulation of temperature is required after effluent temperatures reach $40^{\circ} \mathrm{C}$. We recommend further studies, including expanded testing with the simulation system and behavioral and bioenergetic investigations that may further refine acceptable rates of effluent-temperature increases.
\end{abstract}

\section{Introduction}

We initiated research into the effects of simul ted temperature-rate limits on fish as part of a settlement agreement on fish kills (South Carolina Department of Health and Environmental Control 1990) stemming from the Reactor Operation Environmental Impact Statement (U. S. Department of Energy, 1990). Our primary goal was to minimize thermally related fish kills by providing a sound experimental basis for recommending the rate of power ascension during reactor restart. The data necessary for these recommendations are the effects of the rate of temperature increase and of ambient, or acclimation, water temperature at the time of restart. Although there has been much research into the ecological and physiological interactions of fish and temperature, no previous studies addressed the conditions and fish most impacted by Savannah River Site (SRS) reactor restarts (Paller 1990). The lack of SRS-specific research is why we recommended laboratory studies to determined rates and starting temperatures. The simulation tests dealt specifically with four temperature increase rates and acclimation temperatures and their effects on the young of the year bluegill sunfish (Lepomis macrochirus).

Paller (1990) reported the following on fish behavior in relation to temperature:

"The fish kills on the Savannah River Site are better understood in light of the behavioral responses of fish to various environmental stimuli including temperature, current, and the presence of shelter and forage. Interest in the impacts of electrical-power production on fish has led to a number of field and laboratory studies on the responses of fish to temperature gradients. These studies indicate that fish actively select certain temperatures when given the opportunity. Generally, when presented with a range of temperatures, fish congregate near their preferred temperature and decrease in number toward the upper and lower 


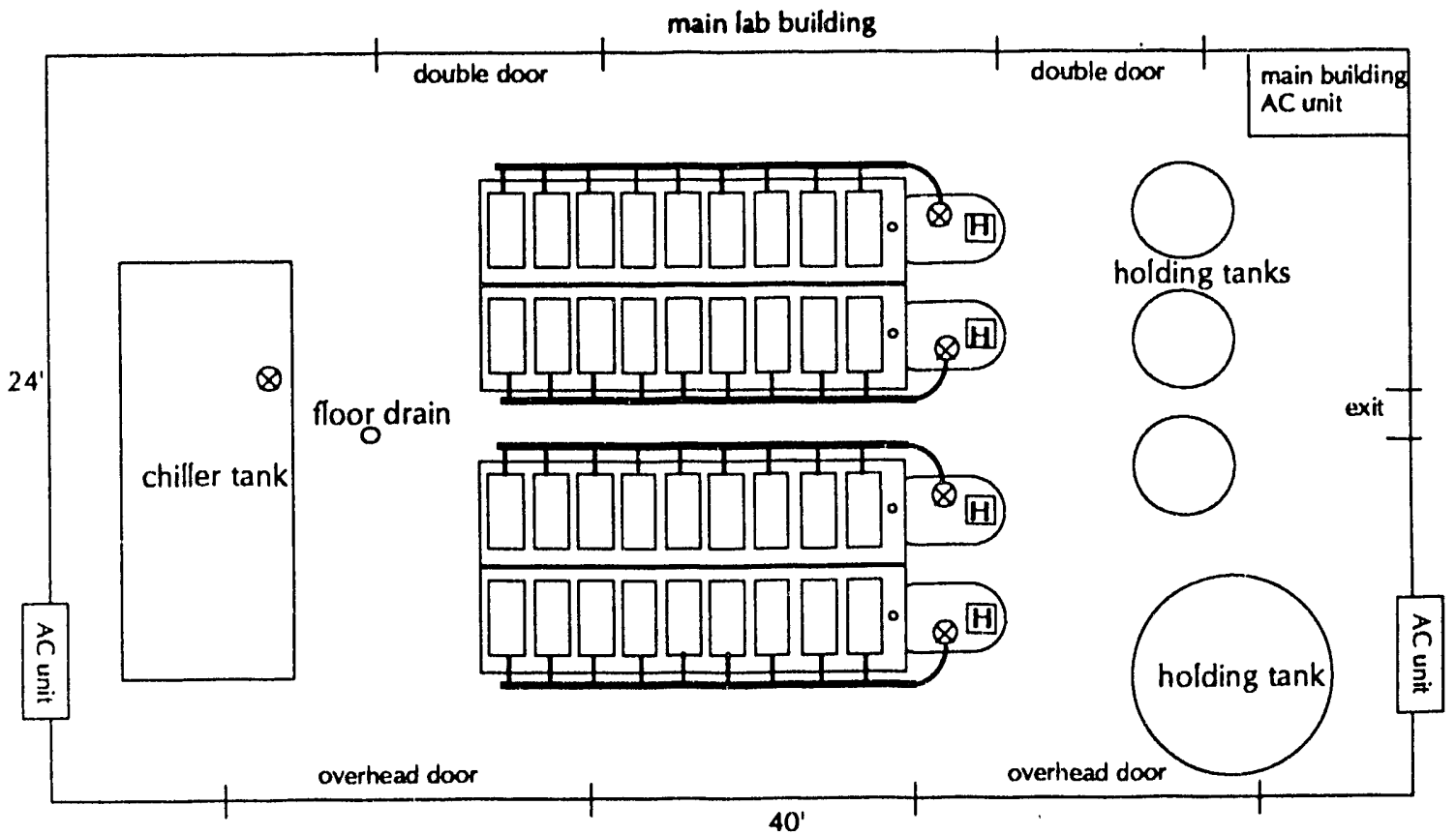

Figure 1. General Layout of the Par Pond Fisheries Laboratory (735-7G)

ends of the temperature gradient (Coutant 1974). There is an upper avoidance temperature above the preferred temperature and a less definite lower avoidance temperature below it. Above the upper avoidance temperature is the upper tolerance limit above which death occurs. The difference between the upper avoidance temperature and the lethal maximum temperature can be as little as 2 to $3^{\circ} \mathrm{C}$, although $5^{\circ} \mathrm{C}$ is not unusual for some species (Brown 1974; Giattina and Garton 1982)."

The thermal history of the fish influences the preferred temperature. Fish held at high temperatures prefer warmer temperatures than those held at low temperatures. This adjustment of temperature preference is called acclimation. However, if given access to a range of temperatures, the preferred temperature of both cold- and warm-acclimated fish will tend to adjust toward a common species (or subspecies) specific value called the final preferendum. This adjustment usually takes several days to complete (Richards et al. 1977). There are limits, however, to the changes in temperature preference and tolerance that can result from acclimation (Coutant 1974; Brown 1974; Giattina and Garton 1982).

Current research into the relationship between fish and temperature has begun to take into account the larger questions concerning the interaction of the energetics of behavioral thermoregulation and the maximum power principle with temperature preference and temperature acclimation (Bryan et al. 1990; Kelsch and Neill 1990).
These directions of research deal with temperature effects on a larger scale and how they affect the physiology and behavior of the organisms and as such become part of the fields of bioenergetics and physiological and behavioral ecology. These aspects of the interactions of fish and temperature and how they are important to the remediation of thermally induced fish kills at SRS is discussed further in the summary.

\section{Methods}

\section{Laboratory Facility}

SRS began developing a wet-lab facility for fisheries research in late June 1990. The west storage bay of the Par Pond Laboratory ( $735-7 \mathrm{G}$ ) was converted to a laboratory by improving electrical service and installing insulation and air conditioning to improve temperature control. The west storage bay was chosen because it had adequate floor space, floor drains, sufficient electrical power, and a direct supply of water from Par Pond. The physical modifications to the storage bay were completed by October 1990, and the final safety inspection was conducted on October 3 . The majority of the required equipment was received by the end of the calendar year. We constructed the simulation apparatus in January 1991. During February, March, and part of April, a series of several different scoping runs were conducted to 


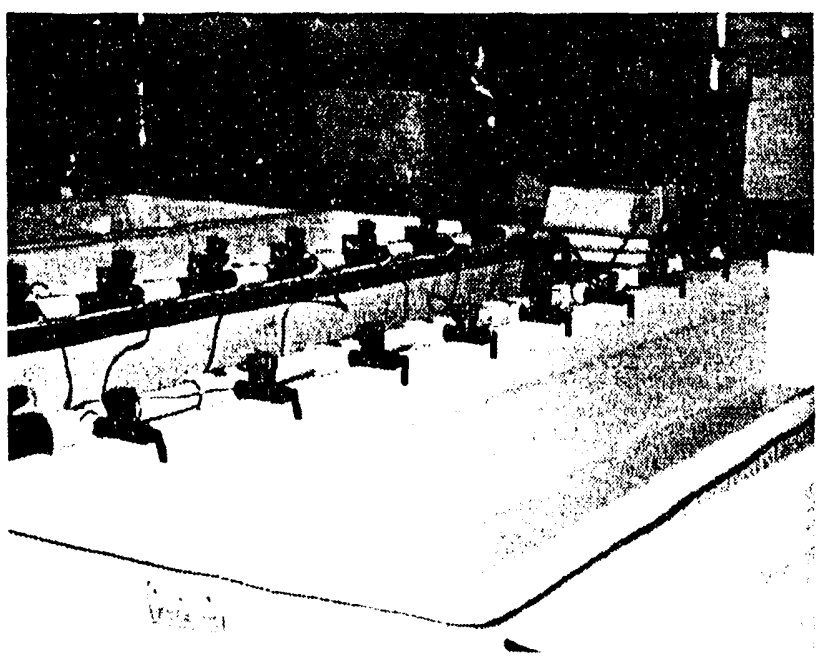

Figure 2. One of Four Troughs I!sed in the Thermal Simulation System

evaluate performance of the system. $A$ general layout of the laboratory is show'n in ligure 1.

\section{Simulation Apparatus}

The simulation apparatus consisted of four similar units; cach had a fiberglass trough $(24.3 \times 5.5 \times 30 \mathrm{~cm})$ situated on a stand (ligure 2) and was supplied by a $20-\mathrm{cm}$. height overflow stand pipe. Fach trough contained g glass aguaria (.39) $\times 20) \times 25 \mathrm{~cm}$ ) (ligure 3) with overflow holes cut in one end. The trough overtlow pipe fed directly into a fiberglass 5() gallon, oval tank by gravity flow. The water in the tank was heated and conled by three 1-KW heatess, and chilled water circulated thromgh stainless sted tubing in the bottom of the tank. A submersible pump recirculated the water to the trough. A manifold and valve system distributed the recirculating water to each of the nine tanks individually. To assure adequate mixing for uniform temperature, a glass tube near the bottom of the tank introduced the water inte the tank. A specially designed IBM XT computer acquired and comtrolled temperature data.

Individual thermocouples made from Omena PRT-24-1000, 24 gage. T-ype thermocouple wire collected temperature data in each of the tanks. The thermocou ples were fed through a $9($ ) degree piece of glass tubing attached to the top cormer of the tank to provide uniform positioning in all tanks. All thermocouples were inputted to Omega l:XP-16 multiplexer boards, then through an ()mega DAS-8 analog-10-digital hoard in the IBM P' ${ }^{\circ}-X T^{\prime}$. Iablech Notebook software was used for temperature control and data acquisition. The system read each thermocouple once a mimute and stored a half-hour block average of the 30) teadings, experiment elapsed time, ait tempera. ture, and tank number on a floppy diskette.

An extra thermocouple in the eighth tank in each trough controlled temperature. The system read the extra thermo couple every 15 seconds and calculated a one-minute rumning average. Bach minute this value was compared to a preset program value dependent on time and desired target temperature, and the heaters were switched accordingly. Heater switching was controlled by an ()mega Pl() 12 digital. 10 interface hoard driving an ()mega I:RB-24 DPIT relay output board The relay board then switched Cole. Parme Dyna-..Sense relay controllers that unned the heaters on and off. cooling water was circulated continuously through the stainless-sted tubing in the 50 gallon tanks lo ohtain water temperatures be low room temperature. A monitor displayed clapsed time, air temperature, and the control temperature of each tromgh. An illustration of the experimental setup appears in ligure 4

\section{Experimental I)esign}

Because two separate factors ane the major contributors to thermally induced death in fish, we conducted two separate experiments. () ne experiment addressed the rate of femperature increase each trough of fish began at the same temperature, and each trough temperature increased at a diflerent rate. Anothere experiment used the same ratte of increase but each trough began with the fish at a different acclimation temperature.

Bothexperiments used small $2.560 .55 \mathrm{~mm}$ ) bhegill sumfish

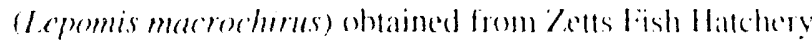

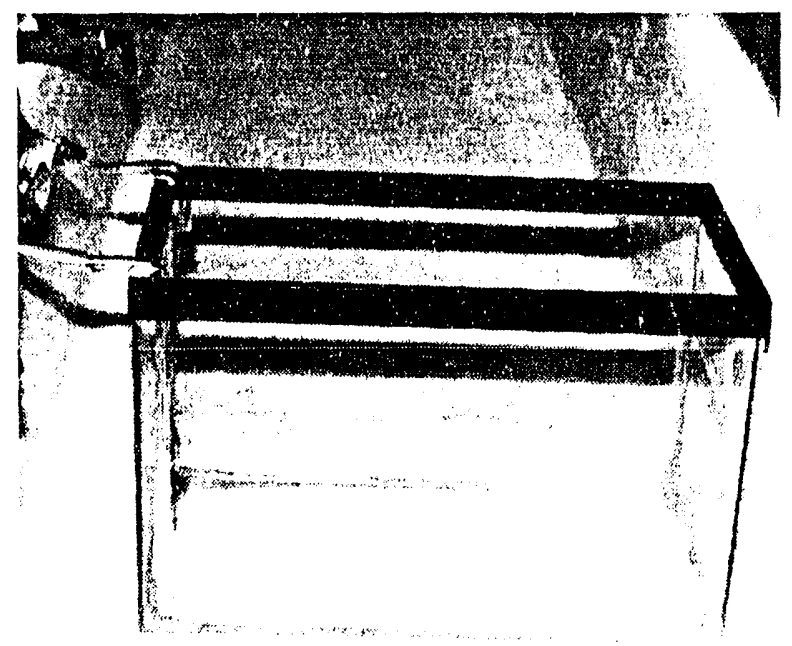

Figure 3. One of 36 Individual Tanks lised in the Thermal Simulation System 


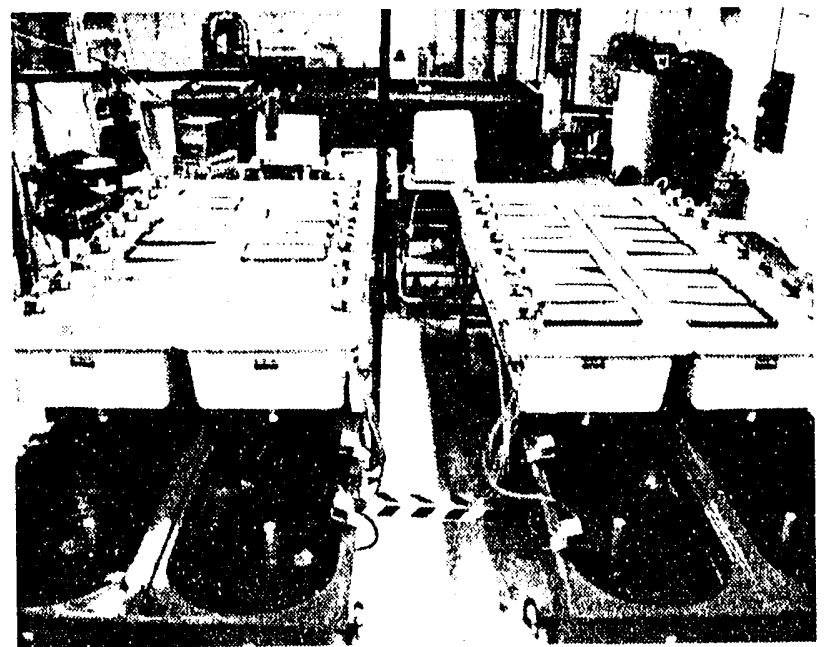

Figure 4. The Thermal Simulation System

in Inwood, West Virginia. We selected this species and size because it is the most vulnerable fish found in potential thermal-kill areas (Paller 1990). Fish were bought from a hatchery to ensure the uniformity and health of the stock and because no immediate means was available for obtaining them from site reservoirs. We limited the number of fish in each tank to 10 per trough, which resulted in a maximum of 90. We acclimated the fish to their starting temperatures for a minimum of five days before the experiment.

Mortality data was collected by direct observation each half hour throughout the experiment. Fish were considered dead when loss of righting ability occurred. Each half-hour observation record included experiment elapsed time, screen-display temperatures, the number of dead fish in each trough, and the number of dead fish in each tank. Figure 5 shows examples of the data sheets. Dead fish were removed from the tanks after they were counted. All recorded data, electronic and observational, was entered into JMP software format for analysis. Mortality-count data were converted to cumulative-percent mortality.

\section{Rate Experiment}

The temperature for the rate experiment began at $20^{\circ} \mathrm{C}$. Trough I was held at 20$)^{\circ}$ ( for the duration of the experiment to serve as a control. 'Troughs 2,3 , and 4 increased by $2.5^{\circ} \mathrm{C}$ increments until they reached $40^{\circ} \mathrm{C}$. Trough 2 increased every 3 hours, Trough 3 increased every 9 hours, and Trough 4 increased every 1.5 hours.

\section{Acclimation Experiment}

The acclimation-effect experiment used a constant increase rate of $2^{\circ} C^{\prime}$ every 12 hours. Trough 1 began at $20^{\circ} C^{\circ}$ and went to $40^{\circ} \mathrm{C}^{\circ}$; Trough 2 began at $15^{\circ} \mathrm{C}$ and went to $39^{\circ} \mathrm{C} \mathrm{C}^{\circ}$ 'Trough 3 began at $25^{\circ} \mathrm{C}$ and went to $39^{\circ} \mathrm{C}$; and trough 4 began at $30^{\circ} \mathrm{C}$ and went to $400^{\circ} \mathrm{C}$

\section{Data Analysis}

A method of comparing the effects of increasing temperature rates on fish mortality fits well into the framework of

\begin{tabular}{l} 
Sample General Data Sheet \\
Day \\
\hline
\end{tabular}

Sample Tank-Specific Data Sheet

\begin{tabular}{l|l|l|l|l|l|l|l|l|l|} 
Trough & Tank & Fish\# & Clip & L In & W In & L Out & W Out & Time of Ieath (Day and Hour) & I)/L \\
\hline & & & & & & & & \\
& & & & & & & & &
\end{tabular}

Figure 5. Examples of General and Tank-Specific Data Sheets 
survival analysis (Dixon and Newman 1991). The exception is that survival analysis is primarily concerned with estimates of the time to death or in estimating the proportion of fish surviving to a given time. Our analysis focuses on the proportion of fish surviving as a function of time, not temperature.

We calculated the number $\left(n_{i}\right)$ of fish at risk of mortality for each $\left(i^{\text {th }}\right)$ steporincrementintargettemperature. Thenumberof deaths $\left(d_{i}\right)$ during the time interval before the next temperature increment also was calculated for each $\left(i^{\text {th }}\right)$ step. Appendix $C$ contains these dataalong with the proportion $\left(q_{i}=d_{i} / n_{i}\right)$ of fish dyinginthe ${ }^{\text {ith }}$ increment.Alifetableanalysiswasperformedusing PROCLIFETEST in the SAS statistical-computing software (SAS 1990).

\section{Results}

\section{System Performance}

We evaluated the performance of the simulation system in maintaining target temperatures by the simple regression of measured temperature on target temperature. The data used were the half-hour block averages recorded by the data acquisition system. The system is capable of heating the water $2^{\circ} \mathrm{C}$ in an hour. Therefore, the two points ( 1 hour) at temperature change were excluded to account for lag time due to thermal inertia of the water.

The regression of actual temperature on target temperature was performed for each of the 63 tanks comprising seven troughs or tests. See Appendix A for a more detailed review of these analyses.

For most of the analyses, regardless of the experiment, these regressions on target temperatures explain $99 \%$ of the variability in the actual temperature measurements. All the regressions are highly statistically significant $(p<0.0001)$.

Estimates of the slope parameters range from 0.91 to 1.07. A slope of 1.0 is the expected value indicating a one-to-one relationship between actual vs target temperatures.

We calculated the precision of the actual temperature measurements given the target temperature as the average of the sum of the squared deviation from the regression line. This is known as the mean squared error (MSE). The square root of this value is an estimate of the standard deviation (SD) of the actual temperature given a target temperature. Table 1 indicates the simulation-system performance. Each value represents 2 SDs, and they indicate that $95 \%$ of all actual temperature measurements occurred within a range of \pm 2 SDs of the target value. As shown in Table 1, these ranges were generally between 1 and $2^{\circ} \mathrm{C}$.

\section{Rate-Effect Experiment}

Our objective for the temperature-increase rates experiment was to evaluate differences in mortality relative to the rate of temperature increase. This information is necessary to support recommended rates of temperature increase to minimize fish kills during reactor restart. For example, if there is no difference in mortality between a rate of $2.5^{\circ} \mathrm{C}$. every nine hours and $2.5^{\circ} \mathrm{C}$ every 15 hours then a recommendation for the slower rate would be unnecessary. Appendix B contains data from the rate-effect experiment for each tank, including elapsed time, target temperature, tank temperature, and percent mortality in one half-hour intervals.

We used the Kaplan-Meier method (Cox and Oakes 1984) for estimating rates in all treatments. Survival rates were high (approximately $90 \%$ ) below $35^{\circ} \mathrm{C}$, but decreased rapidly over the higher temperatures with complete or near-complete mortality by $40^{\circ} \mathrm{C}$. The statistical analysis showed that survivorship with increasing temperature was significantly different $(\mathrm{p}<0.001)$ among the three different rates. Further analysis showed no difference between the two slower rates of temperature increase (Tests 2 and 3), but a significant difference was seen between Tests 1 and 2 and between Tests 1 and 3 . This indicates the highest increaserate test had a different survival rate than the other two increase rates. Examination of the curves (Figure 6) shows that test 1 , the highest increase rate, had lower survival than the other two rates. Figure 6 is a plot of the survivorship curves for these tests and also includes data from the $20^{\circ} \mathrm{C}$ acclimation temperature trough (shown as Test 4 ) from the acclimation-effect experiment discussed next.

In Figure 6, Tests 1, 2, and 3 are from the rate experiment and were compared statistically. Test 4 is the $20^{\circ} \mathrm{C}$ acclimation trough from the acclimation experiment and was included only for visual comparison not statistical analysis. Statistically, Test 1 is not similar to Tests 2 or 3 , but Tests 2 and 3 are similar. These curves represent survivorship associated with temperature-increase rates of 2.5 degrees every 3,9 , and 15 hours. The results indicate that at the 20-degree acclimation temperature at which these tests were conducted, no significant reduction in mortality would be gained by using a 15-hour step instead of a nine hour step. Significant gains are available by using nine hours instead of three for the increase interval. The line from Test 4 , representing the $20^{\circ} \mathrm{C}$ acclimation trough from the acclimation experiment, shows that up to approximately $37^{\circ} \mathrm{C}$ a rate increase of $2^{\circ} \mathrm{C}$ every 12 hour rate of increase 
Table 1. Simulation System Performance as indicated by $95 \%$ confidence interval of actual temperature around the target temperature.

Acclimation Experiment

Deviation from Target

Temperature $\left(+/-{ }^{\circ} \mathrm{C}\right)$
Rate Experiment

Deviation from Target

Temperature $\left(+/{ }^{\circ} \mathrm{C}\right)$

Trough 1

Tank

$\begin{array}{ll}1 & .62 \\ 2 & .62 \\ 3 & .59 \\ 4 & .60 \\ 5 & .62 \\ 6 & .56 \\ 7 & .52 \\ 8 & .62 \\ 9 & .60\end{array}$

Trough 2

Tank 10

.86

1.14

.86

1.12

.86

1.04

13

.86

1.08

.86

1.10

.72

.66

1.08

1.02

.76

1.10

18

.66

1.16

Trough 3

Tank 19

.86

.80

20

.62

.70

21

.66

.76

22

.70

.72

23

24

25

26

27

.56

.56

.52

.52

.54

.80

.70

.74

.66

Trough 4

Tank 28

.66

.64

.58

.60

.56

.58

.56

.60

.68

.98

1.00

.96

1.02

1.00

1.00

.96

1.10

1.02 


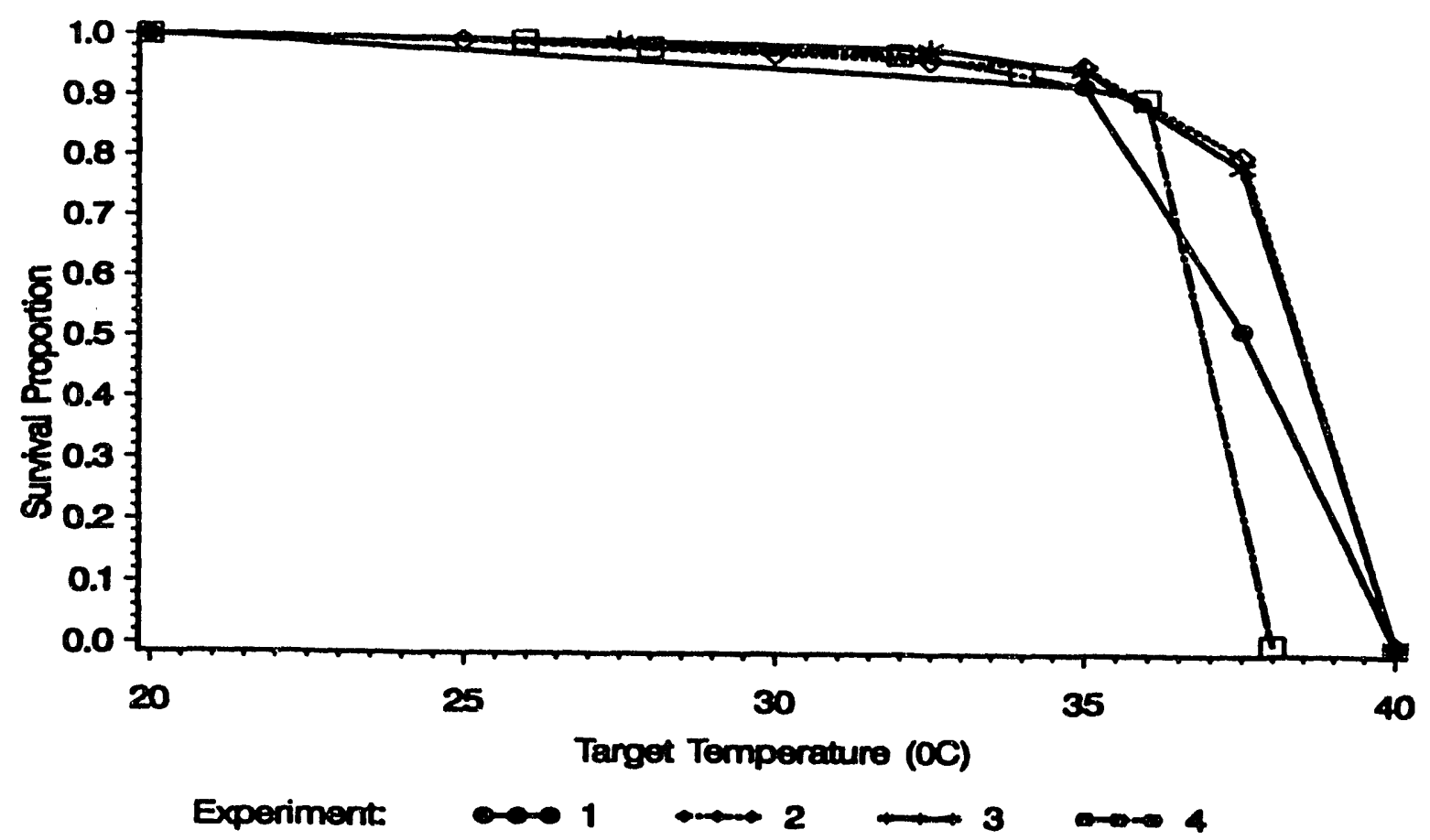

Figure 6. Survivorship Curves for Rate Experiment

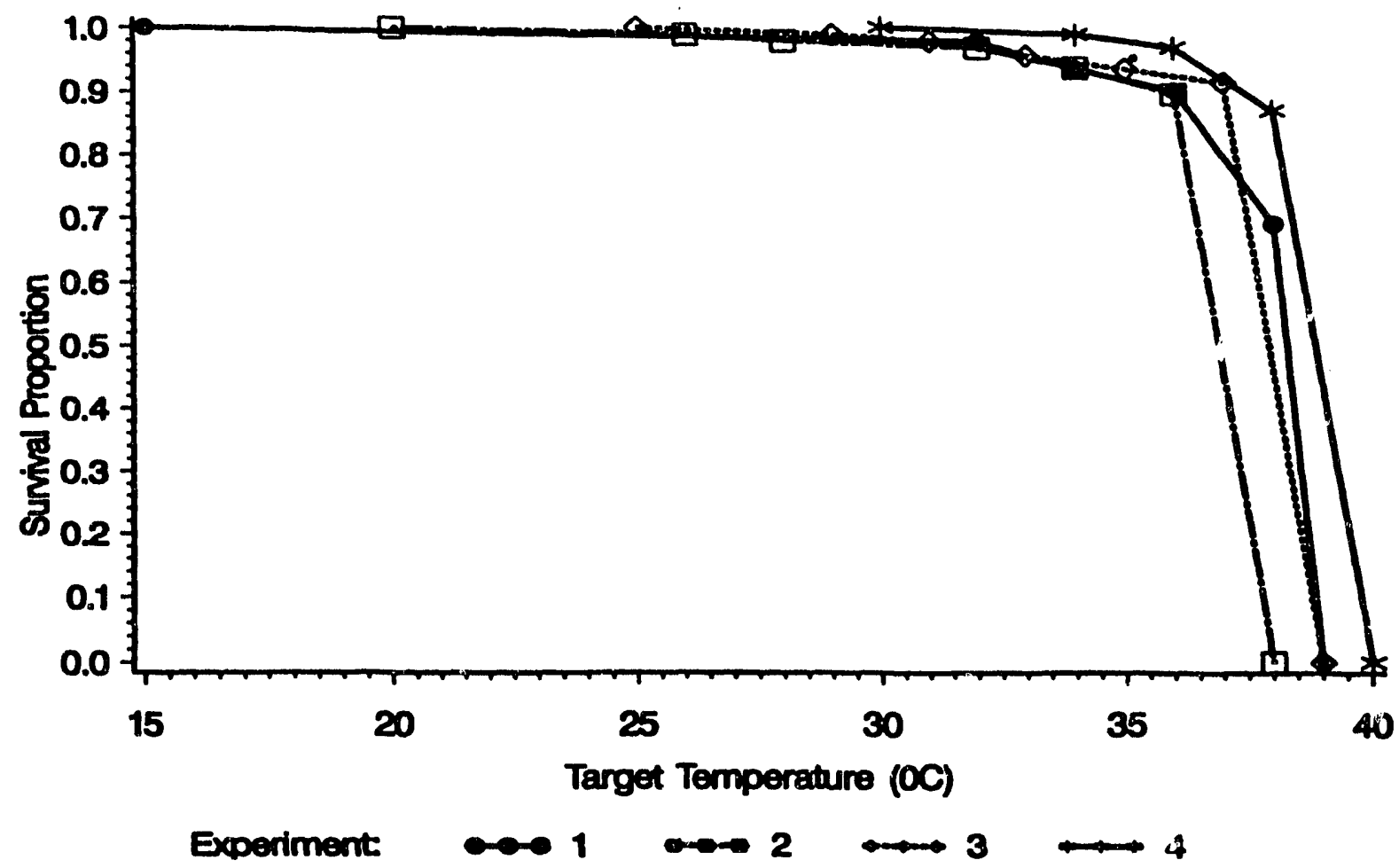

Figure 7. Survivorship Curves for Acclimation Experiment 
will have similar survivorship to the rates of 2.5 every nine and 15-hour. The precipitous drop in survivorship to zero by $38^{\circ} \mathrm{C}$, lower than any of the tests in either the rate or acclimation experiments, is unexplained.

\section{Acclimation-Effect Experiment}

During early scoping runs to set up and test the thermal simulation apparatus, mortality appeared to be affected by the acclimation temperature. We found this consistent with other studies (for example, Peterson and Schutsky 1976) that shov:ed an increase in lethal temperature with higher acclimation temperature. We conducted an experiment to test the effect of different starting acclimation temperatures. The acclimation temperatures in the four troughs were different $\left(15,20,25\right.$, and $\left.30^{\circ} \mathrm{C}\right)$, but the rate of temperature increase was a constant $2^{\circ} \mathrm{C}$ every 12 hours. Appendix B lists the data from the acclimation-effect test for each tank, including elapsed time, target temperature, tank temperature, and percent mortality in half-hour intervals.

We performed a life-table analysis to compare the effects of acclimation temperature on fish mortality as a function of increasing water temperature. The analysis showed that survivorship was significantly different $(p<0.001)$ among the four tests. Further comparison of pairs showed that no two survivorship curves were similar (Figure 7). This indicates that survivorship is significantly affected by differences in acclimation temperature.

Survivorship decreases least rapidly among fish acclimated to the highest initial temperature of $30^{\circ} \mathrm{C}$ (Test 4). The $15^{\circ} \mathrm{C}$ test had the most rapid decline in survivorship, and the $20^{\circ} \mathrm{C}$ test had the lowest temperature for zero survivorship.

These results indicate that the ambient temperature of the water, which is controlled by season, should be considered before establishing temperature ascension rates. When the water temperature is $20^{\circ} \mathrm{C}$ or below, SRS should slow the rate of temperature increase to account for the risk associated with lower acclimation temperatures.

The slower rate of increase helps in two ways. First, it allows for a longer period of time before temperatures reach critical levels. Second, even though it does not afford adequate time for the fish to acclimate to the higher temperatures, the slower rate allows more time for physiological adjustment by the fish.

\section{Summary and Conclusions}

Based on this study, we concluded that modifying the rate at which reactor power and discharge temperatures are increased during startup can reduce the magnitude of thermally related fish kills. Analysis of Figures 6 and 7 provides insight into how temperature-ascension rates can be modified to minimize thermally induced fish kills. The curve that extends most horizontally before the precipitous drop has the most fish surviving to a higher temperature. Likewise, a curve that has more distance along the temperature axis between the initial drop in survivorship and the final point affords the longest time for escape. The rate experiment shows no difference in survivorship between rates of $2.5^{\circ} \mathrm{C}$ every 9 and 15 hours, but significantly lower survivorship at $2.5^{\circ} \mathrm{C}$ every 3 hours. The acclimation experiment shows differences in survivorship for each separate acclimation temperature, examination of the curves in Figure 7 indicates lower survivorship for lower acclimation temperatures.

Based on these results, when ambient water temperature is above $20^{\circ} \mathrm{C}$, a temperature increase rate of $2.5^{\circ} \mathrm{C}$ every nine hours is adequate to minimize mortality. However, when water temperatures are $20^{\circ} \mathrm{C}$ or lower, a slower rate of increase is desirable. This research does not provide a statistical basis for this recommendation, but best scientific judgment and interpretation of the data suggest that a slower rate would be beneficial. We suggest an increase rate of $2.5^{\circ} \mathrm{C}$ every 12 hours when ambient water temperature is $20^{\circ} \mathrm{C}$ or lower

\section{Options for Additional Studies}

The data supporting this report represent a substantial gain in understanding how reactor-restart temperature increases affect fish mortality in the discharge area and contribute to methods that help mitigate mortality. However, there are other areas where further research could significantly enhance our understanding of the forces at work and contribute to further mitigation of fish kills. This research could be conducted with equipment that we have and a minimal investment of time.

The first area should be a direct extension of the work already conducted. We could significantly refine of the current recommendations by using the current data and modifying the experimental procedures to address narrower questions regarding the relationship of acclimation tem- 
perature, temperature increase rate, and fish survivorship. This could provide reactor operators with a precise method for maximizing effluent-temperature increase rates under any environmental conditions while maintaining an acceptable level of fish mortality. A response surface-design experiment (Cochran and Cox 1957) appears to be a strong candidate to provide this information.

Because recommended temperatures and rates would be controlled at the point of discharge, known effects of the temperature regimen from the simulation research would only be valid as far as the discharge-canal mouth. Distribution of the thermal effluent due to overrunning and other hydrodynamic effects and their impact on the fish community would be very difficult to determine. We find it desirable instead to examine habitat use by fish of at-risk size during normal and reactor start up conditions.

Fish have a variety of behavioral responses to increasing temperatures. Fish are attracted to areas where the temperature is increasing toward the preferred temperature; however, as temperature continues to rise into the avoidance range, behavior changes as the fish seek to escape the effects of the increase. This is when fish, especially the smaller ones, tend to be aggregated in shallow areas near the shore where the increasing temperature prevents their escape to deeper, cooler water.

Shoreline recontouring of the upper part of $L$ Lake may help reduce these effects (Paller 1990), but a detailed examination of habitat use under normal and startup conditions will better define any necessary recontouring. This may involve a cost savings because shoreline recontouring is expensive and time consuming. We can conduct the habitat use experiment with sophisticated radiotelemetry equipment, which the Environmental Sciences Section (ESS) Ecology Group is currently testing.

Another area of recommended research deals with fish behavior and physiology, which are inextricably tied to thermally-induced mortality. We need to investigate these areas because it is probable that even if temperature-increase rates were held to very conservative values, significant levels of mortality might occur due to behavioral and bioenergetic effects linked to the change in temperature. These mortalities would not be directly caused by the increased temperature, but would be a function of energetic depletion due to extended periods of avoidance behavior and long-term residence by the affected organisms in water of sublethal temperature water.

Behavioral thermoregulation of fish in heterogeneous thermal environments is largely dictated by the maximum power principle and the accompanying maximization of

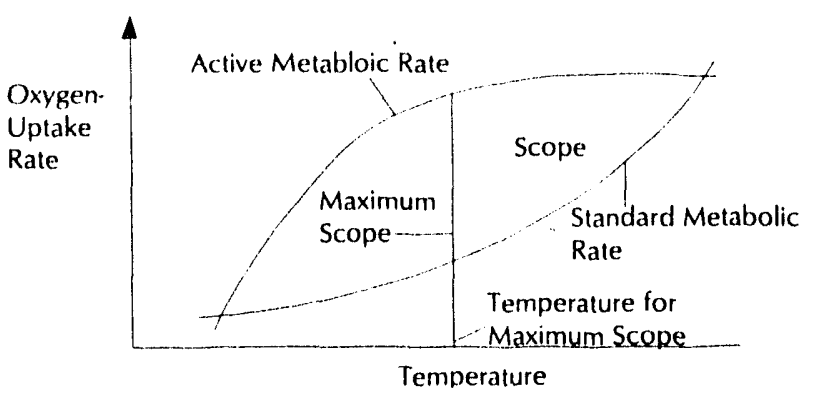

Figure 8. Scope for Activity (Kelsch and Neill 1990)

useful energy by the organism (Bryan et al. 1990). By avoiding lethal temperatures and remaining in preferred temperature areas, fish minimize thermoregulatory swimming effort, which maximizes power available for growth, reproduction, and energy acquisition.

When a fish is forced into an undesirable environment by introducing large amounts of high-temperature water, its maximum scope for activity is reduced (i.e., forced to the right in Figure 8). This decrease in metabolic scope is a real decrease in available power, and the fish responds with a change in behavior. This change in behavior increases swimming speed and energy expenditure. In an effluentdominated environment, the organism will not find thermal conditions near the preferred temperature and will not return to more energy-efficient behavior. These behavioral changes mean the fish will eventually exhaust its energy reserves, lose the capacity for directed movement, and eventually die because of the increasing temperatures or a sort of "induced starvation". Smaller fish are more susceptible to this effect because a larger size increases survival time under starvation conditions (Shuter et al. 1989). Therefore, larger fish could sustain avoidance behavior without feeding for a longer time than small fish, which gives them a greater potential for escaping the effects of the thermal effluent.

Further research is needed to quantify exactly how long and at what temperature fish in the discharge area can survive and engage in avoidance behavior before this bioenergetic death occurs. It is possible that power-ascension rates can be increased more rapidly or in larger temperature blocks without significantly increasing mortality rates.

Two specific areas of research can help address the behavioral and bioenergetic questions. Digitized videobased behavioral analysis equipment currently being tested in the ESS fish lab at Par Pond can determine avoidance temperatures of fish acclimated to different temperatures. This knowledge may allow much higher rates of temperature increase up to the avoidance temperature. The guidance 
temperature could be held for a predetermined period of time to allow the maximum number of fish to escape the effluent area. The time period for tilis avoidance temperature hold can be determined empirically in the laboratory by determining the avoidance temperature and then holding fish at that temperature until dying due to energy depletion. The time between initial avoidance temperature and death would be the maximum time to hold effluent temperature at the avoidance temperature. After the hold time, effluent temperature could be raised quickly to its maximum value. In addition, after this "bioenergetic starv ation". the fish can be freeze-dried and its whole-body-energy content determined by oxygen-bomb calorimetry. These energy values, when compared to fish not exposed to increased temperatures and fish killed by rapid temperature increase, will allow better understanding of the bioenergetic dynamics involved in the restart-related mortality and may allow for easier and more cost-effective means of reducing that mortality.

It is not likely that any research will provide information that will eliminate fish mortality during restart because the size of the affected area and the high final temperatures essentially preclude the successful escape of all fish in the effluent area. The relatively small size of the most vulnerable fish and the large heated-discharge area are finite parameters that cannot be easily altered and appear to be primary factors controlling the extent of fish mortality during reactor restart. However, further research can contribute to more exact and cost-effective mitigation measures.

\section{Acknowledgments}

The technical analyst staff of ESS contributed much of its time to the collection of data, and W. M. Fulmer individually carried significant responsibility for the set up and smooth operation of the laboratory and coordination of the experimental runs. W. J. Macky (Lab Services, E\&I) provided assistance in the initial setup of the lab. Chas Murphy, Jr. of ESS helped with the initial installation and test of the data acquisition and process-control system hardware and software. Mike Paller of ESS provided advice and background fisheries information.

\section{References}

Brown, H. W., 1974, "Handbook on the effects of temperature on some North American fishes", American Electric Power Service Corporation, Canton, $\mathrm{OH}$.

Bryan, J. D., S. W. Kelsch, and W. H. Neill, 1990, "The Maximum Power Principle in Behavioral Ther- moregulation by Fishes", Trans. Am. Fish. Soc., 119:611-621

Cochran, W. G., and G. M. Cox, 1957, Experimental Designs, 2nd ed., John Wiley and Sons, Inc., New York, NY, $611 \mathrm{pp}$

Coutant, C. C., 1974, "Temperature selection by fish - a factor in power-plant impact assessments", IAEASM-187/11, Environmental Sciences Division, Oak Ridge National Laboratory, Oak Ridge, TN

Dixon, P. M., and M. C. Newman, 1991, Analyzing Toxicity Data Using Statistical Models for Time-to-Death: An Introduction in: Metal Ecotoxicology, Concepts and Applications, Edited by Michael C. Newman and Alan W. McIntosh, Lewis Publishers, Inc. Chelsea, MI, 339 pp

Giattina, J. D., and R. R. Garton, 198?, "Graphical model of thermoregulatory behavior by fishes with a new measure of eurythermality", Can. J. Fish. Aquat. Sci., 39:524- 528

Kelsch, S. W., and W. H. Neill, 1990, "Temperature Preference versus Acclimation in Fishes: Selection for Changing Metabolic Optima", Trans. Am. Fish. Soc., 119:601-610

Paller, M. H., 1990, A Remedial Astion Plan for Fish Kills in L Lake and Pond C, WSRC-RP-90-473, Rev. 0, Westinghouse Savannah River Company, Savannah River Site, Aiken, South Carolina.

Peterson, S. E., and R. M. Schutsky, 1976, "Some relationships of Upper Thermal Tolerances to Preference and Avoidance Responses of the Bluegill", In G. W. Esch, and R. W. McFarlane, eds., Thermal Ecology II. ERDA symposium series, CONF-750425

Richards, F. P., W. W. Reynolds, and R. W. McCauley, 1977, "Temperature preference studies in environmental impact assessments: an overview with procedural recommendations", J. Fish. Res. Board Can., 34:728-761

SAS Institute Inc., 1990, SAS/STAT User's Guide, Vol. 2, Release 6.06 Edition, Cary, NC, pp 1027-169

South Carolina Department of Health and Environmental Control, 1990, "Settlement Agreement \#90-26-W", Fish Kill Issue

Shuter, B. J., P. E. Ihssen, D. L. Wales, and E. J. Snucins, 1989, "The effects of temperature, $\mathrm{pH}$, and water hardness on winter starvation of young-of-the-year smallmouth bass, Micropterus dolomieui Lacepede", J. Fish Biol., 35:765-780

U. S. Department of Energy, 1990, "Final Environmental Impact Statement" Continued Operation of K-, L-, and P-Reactors, Savannah River Site, Aiken, South Carolina", DOE/EIS-0147

Cox, D. R. and D. Oakes, 1984, Analysis of Survival Data. Chapman and Hall, London. 
Appendix A 


\section{Acclimation Experiment Tanks 1-4}
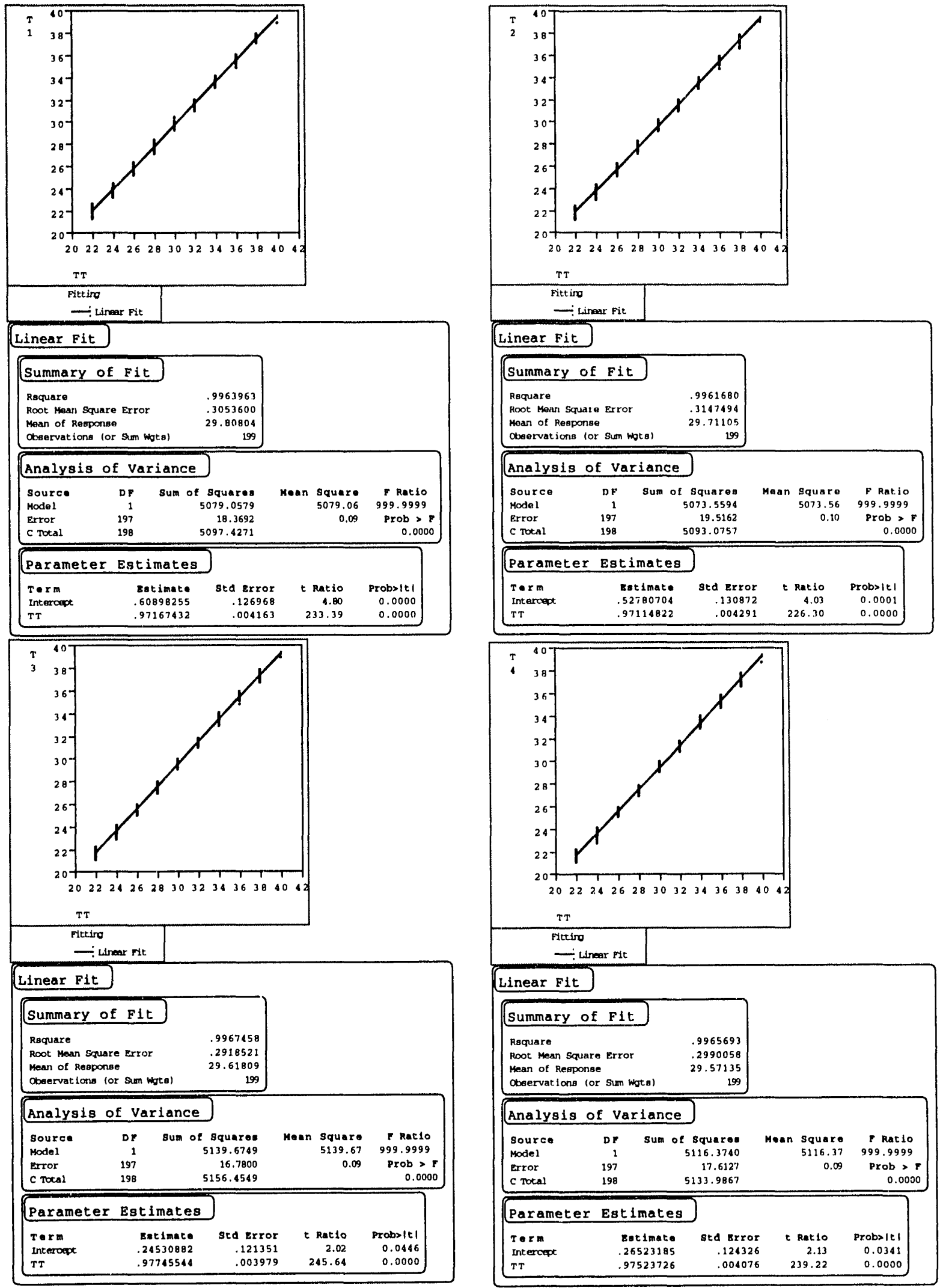


\section{Acclimation Experiment Tanks 5-8}
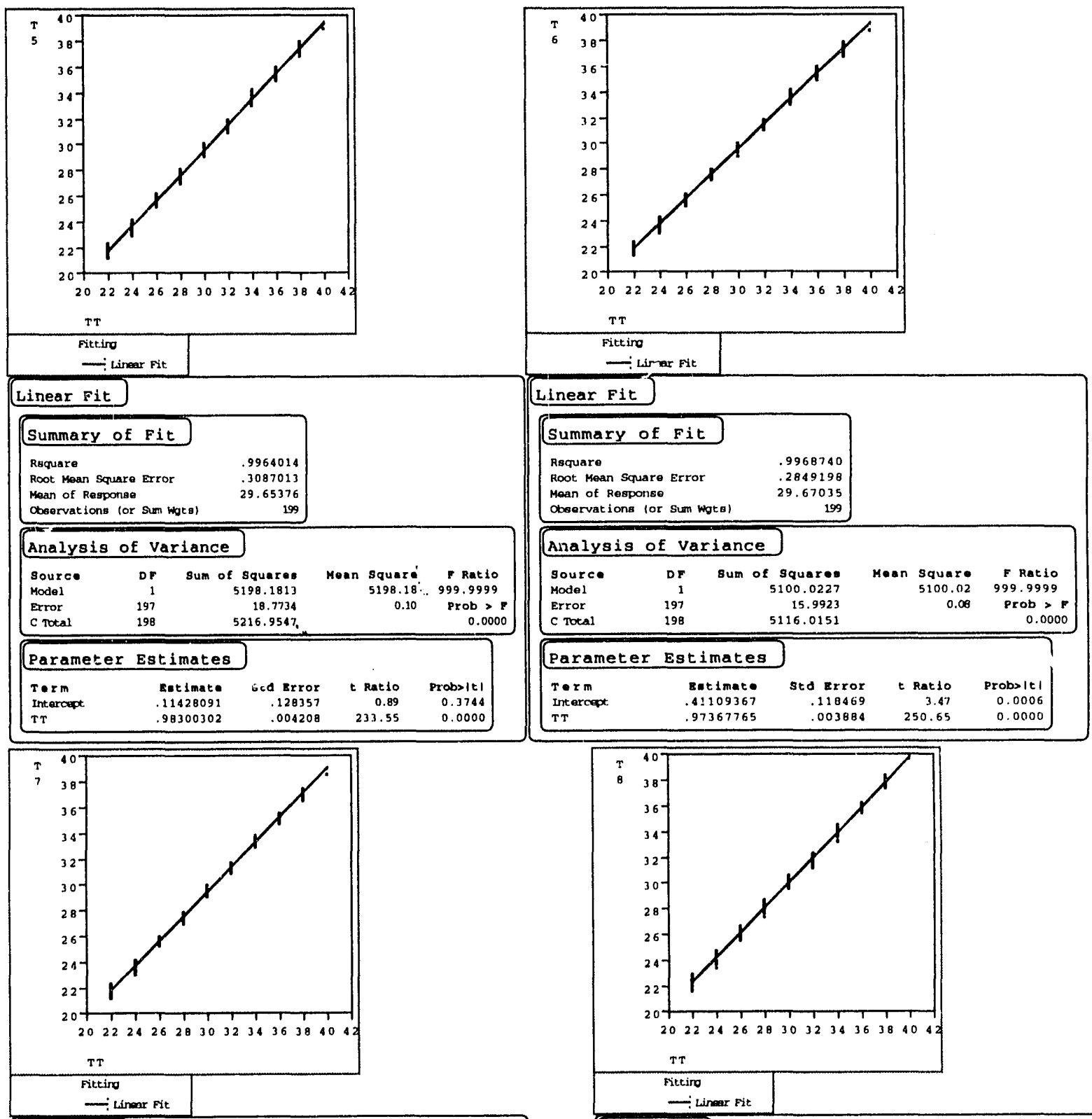

Linear Fit
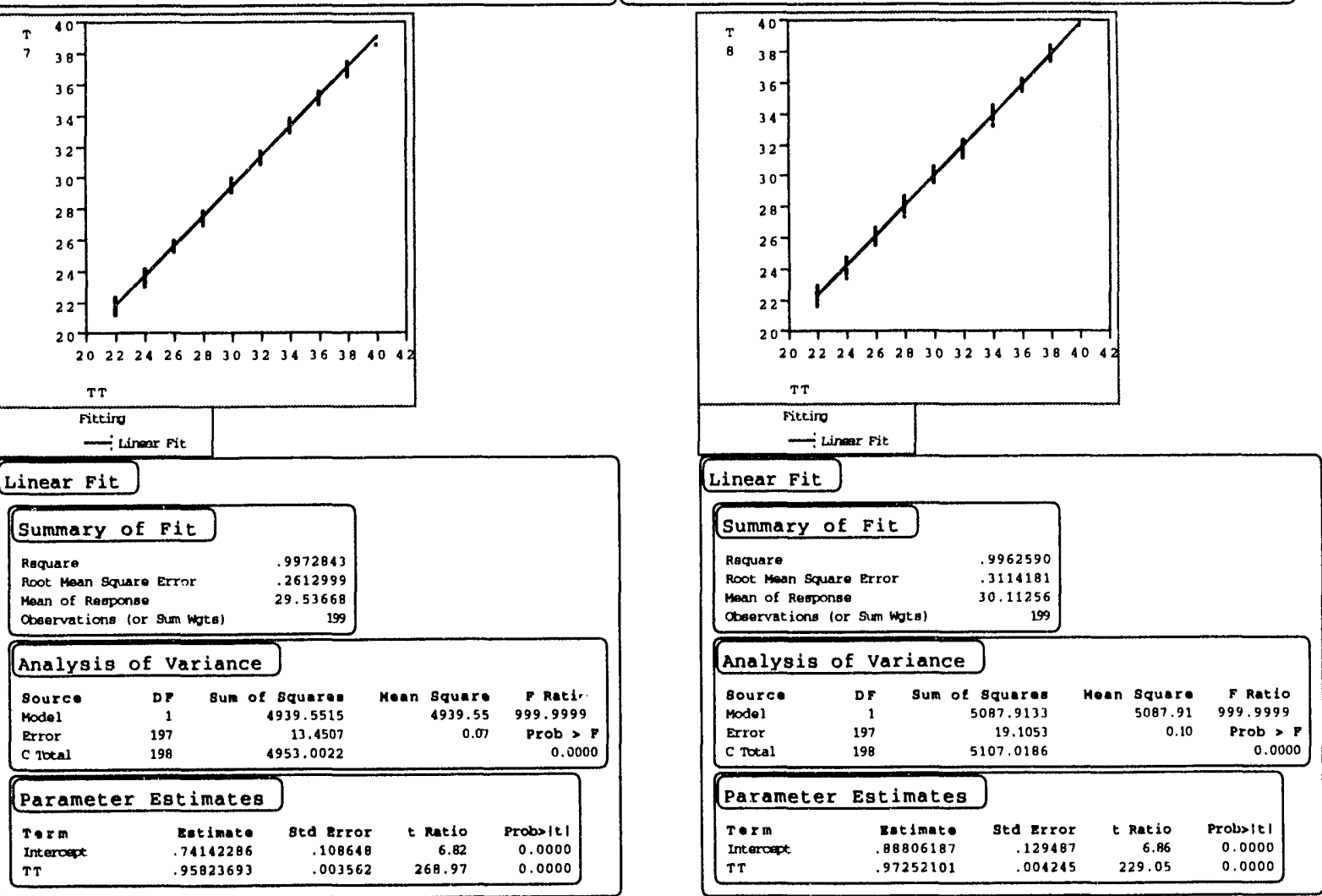


\section{Acclimation Experiment Tanks 9-12}
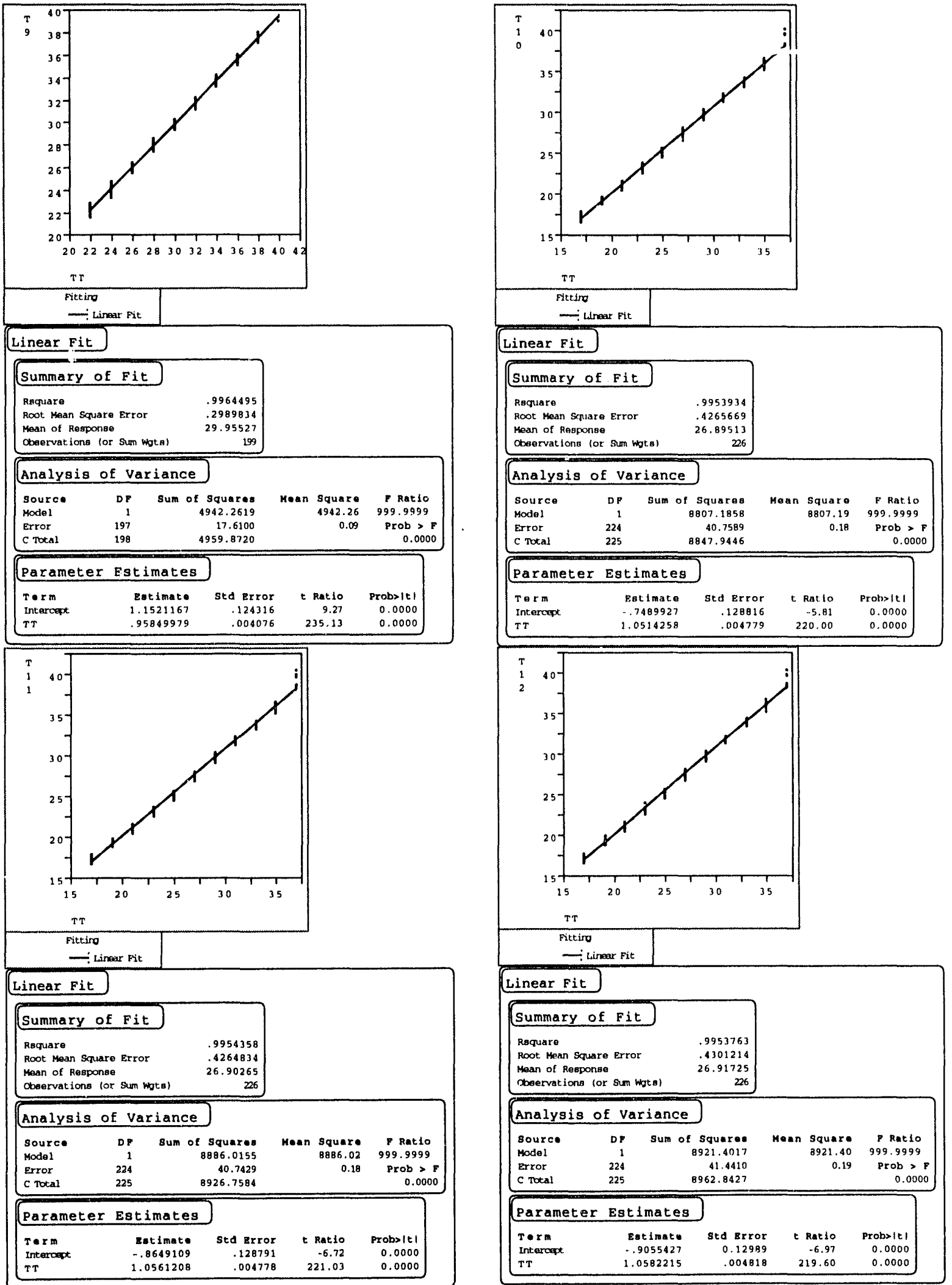


\section{Acclimation Experiment Tanks 13-16}
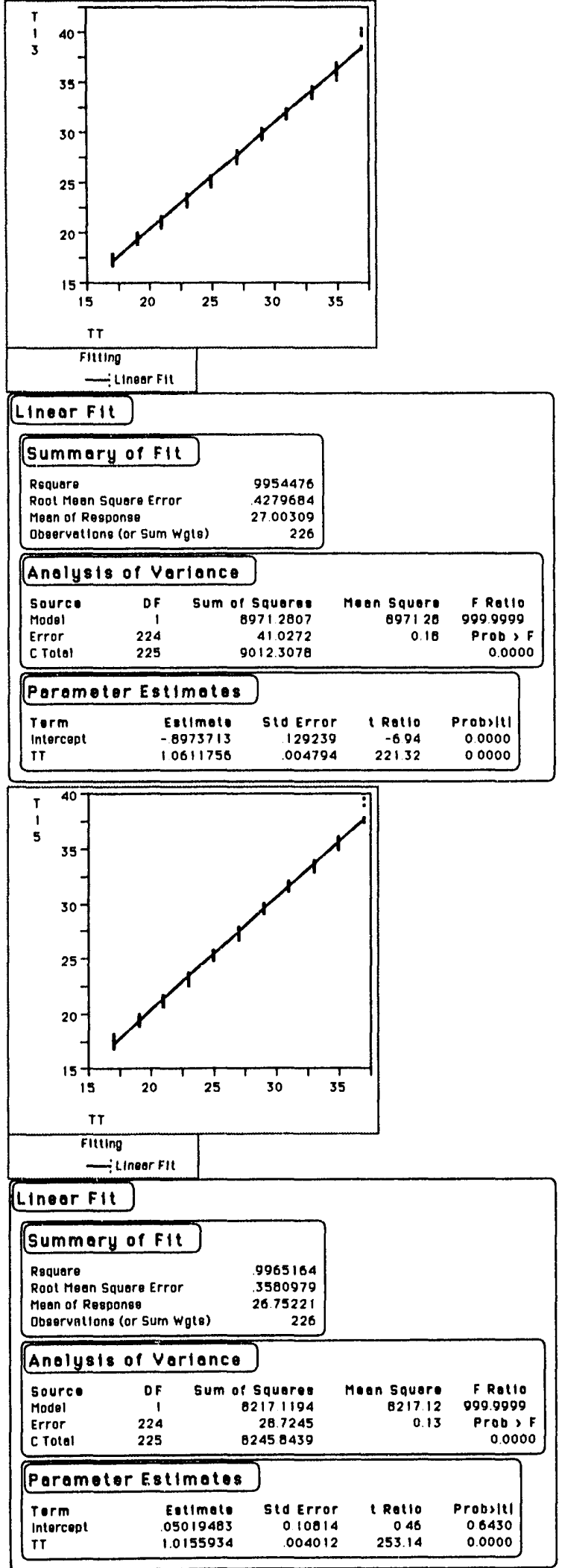
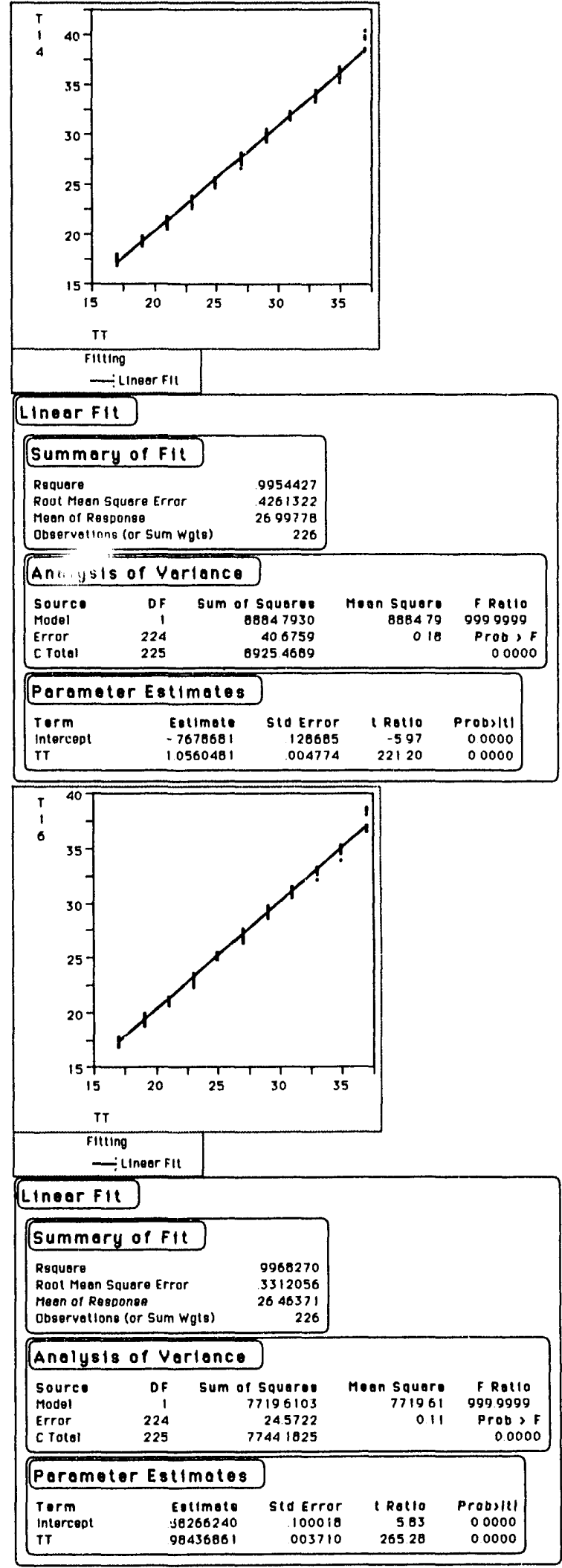


\section{Acclimation Experiment Tanks $\mathbf{1 7 - 2 0}$}
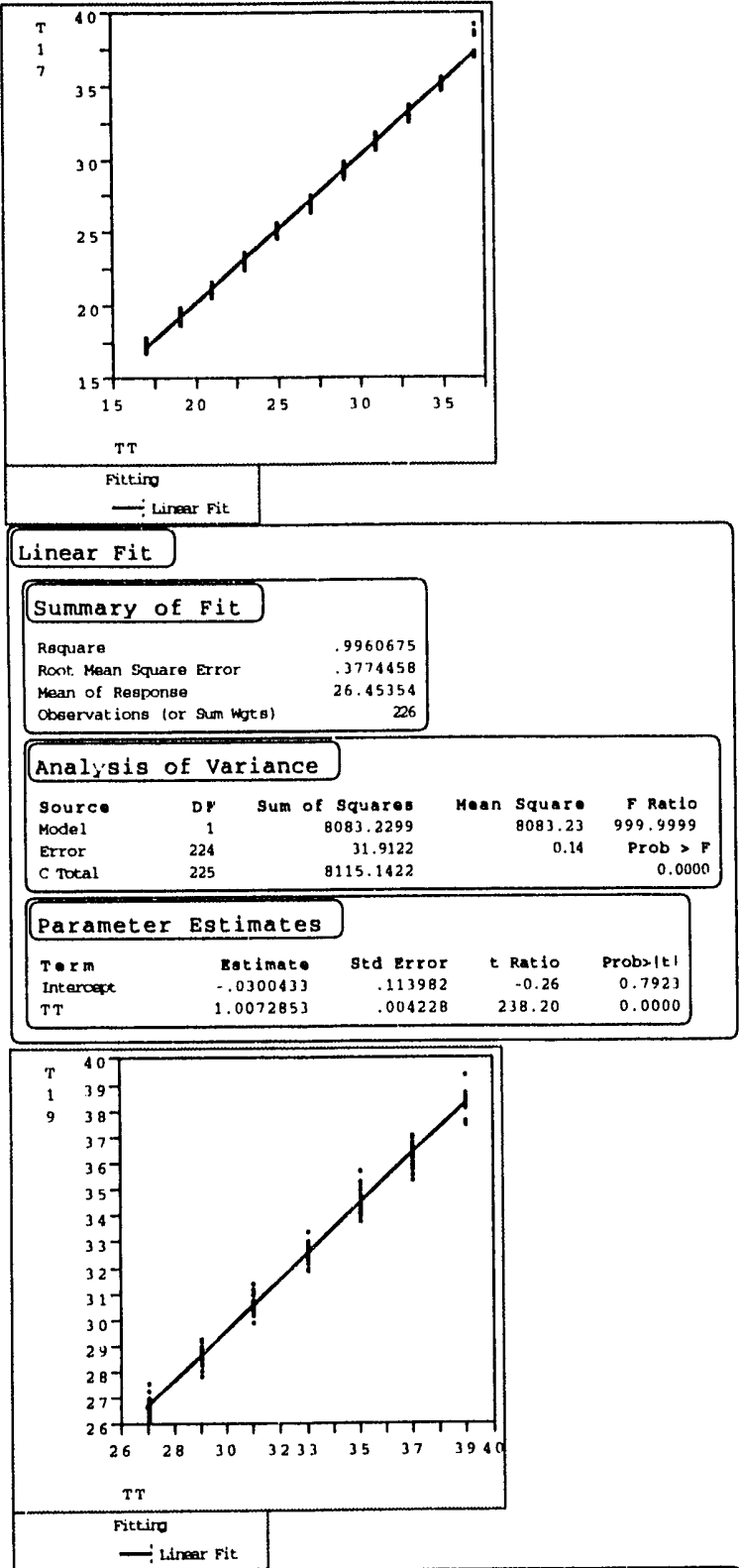

Linear Fit

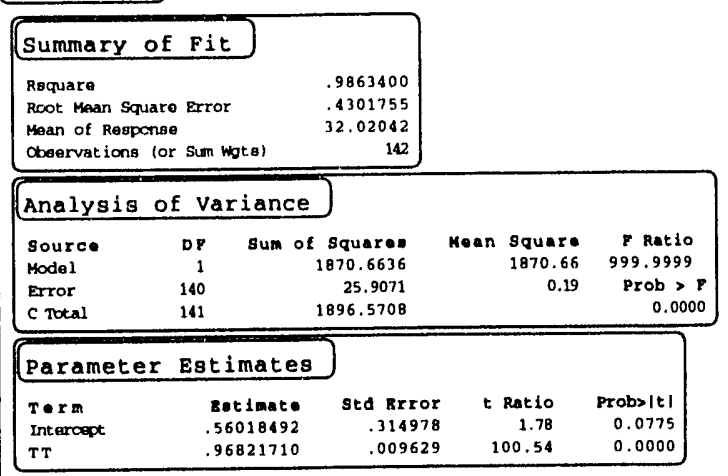
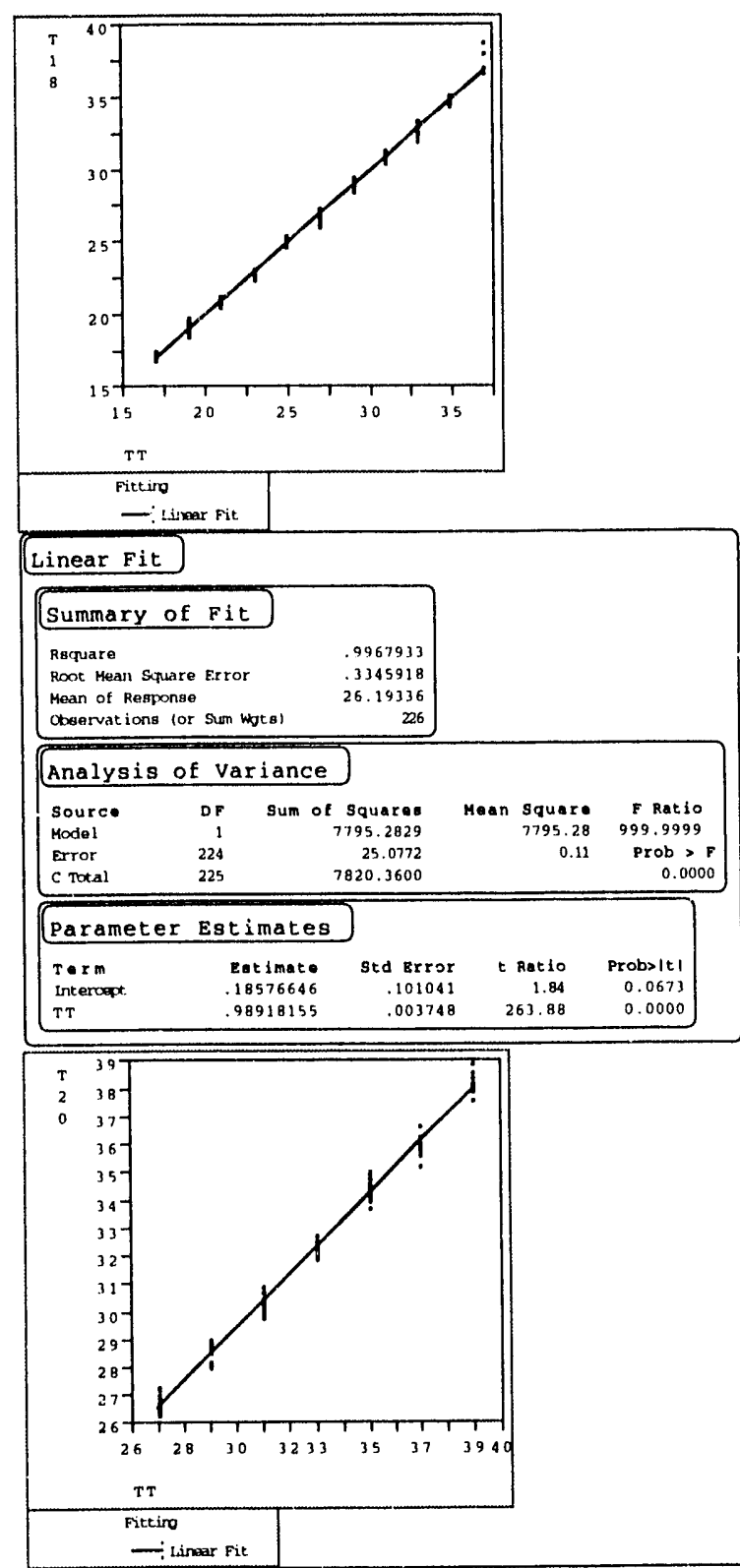

Linear Fit

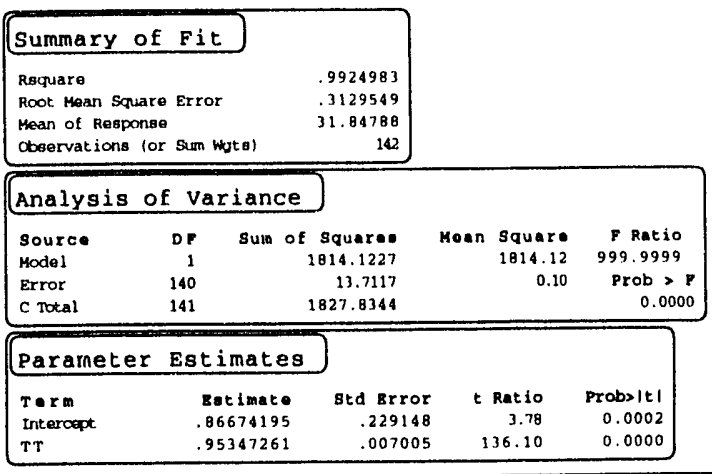




\section{Acclimation Experiment Tanks 21-24}
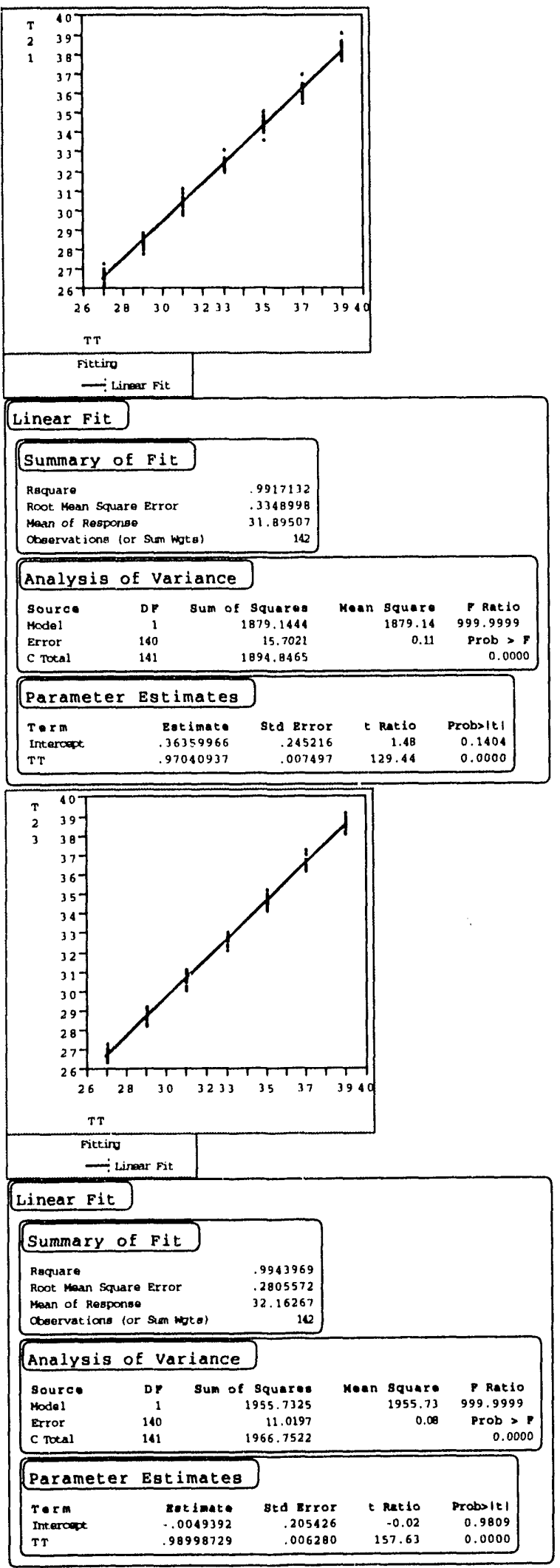
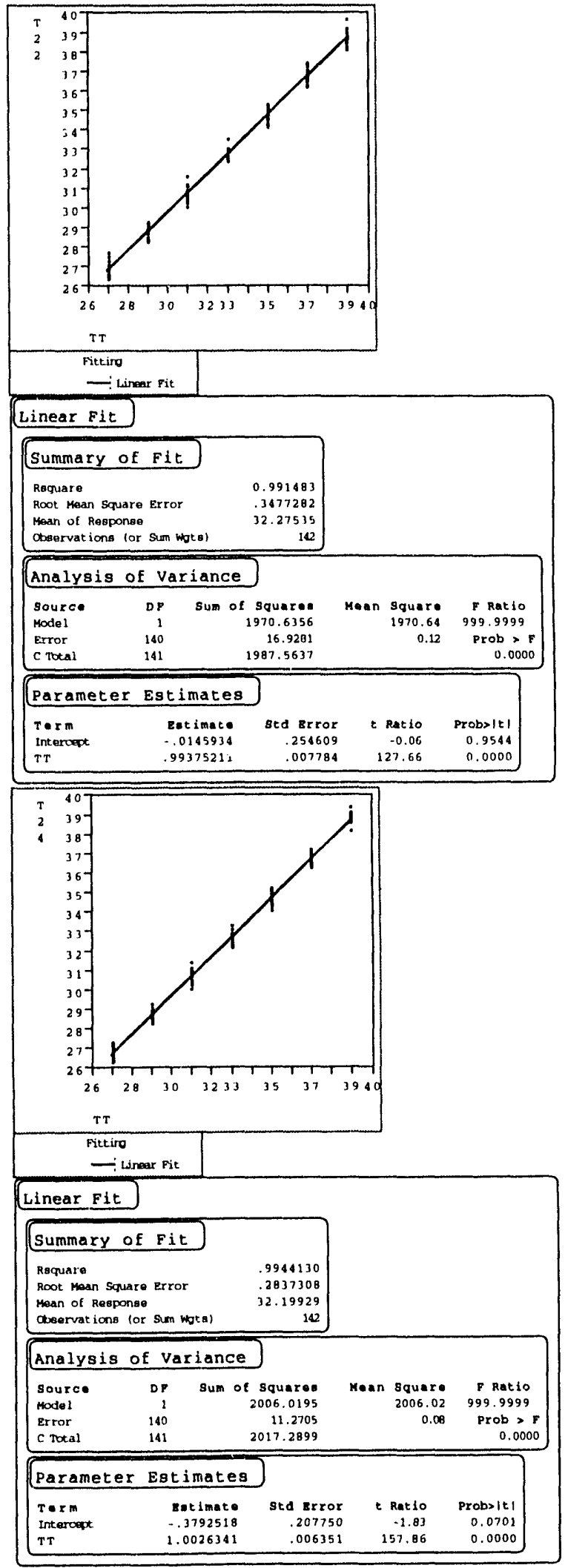


\section{Acclimation Experiment Tanks 25-28}
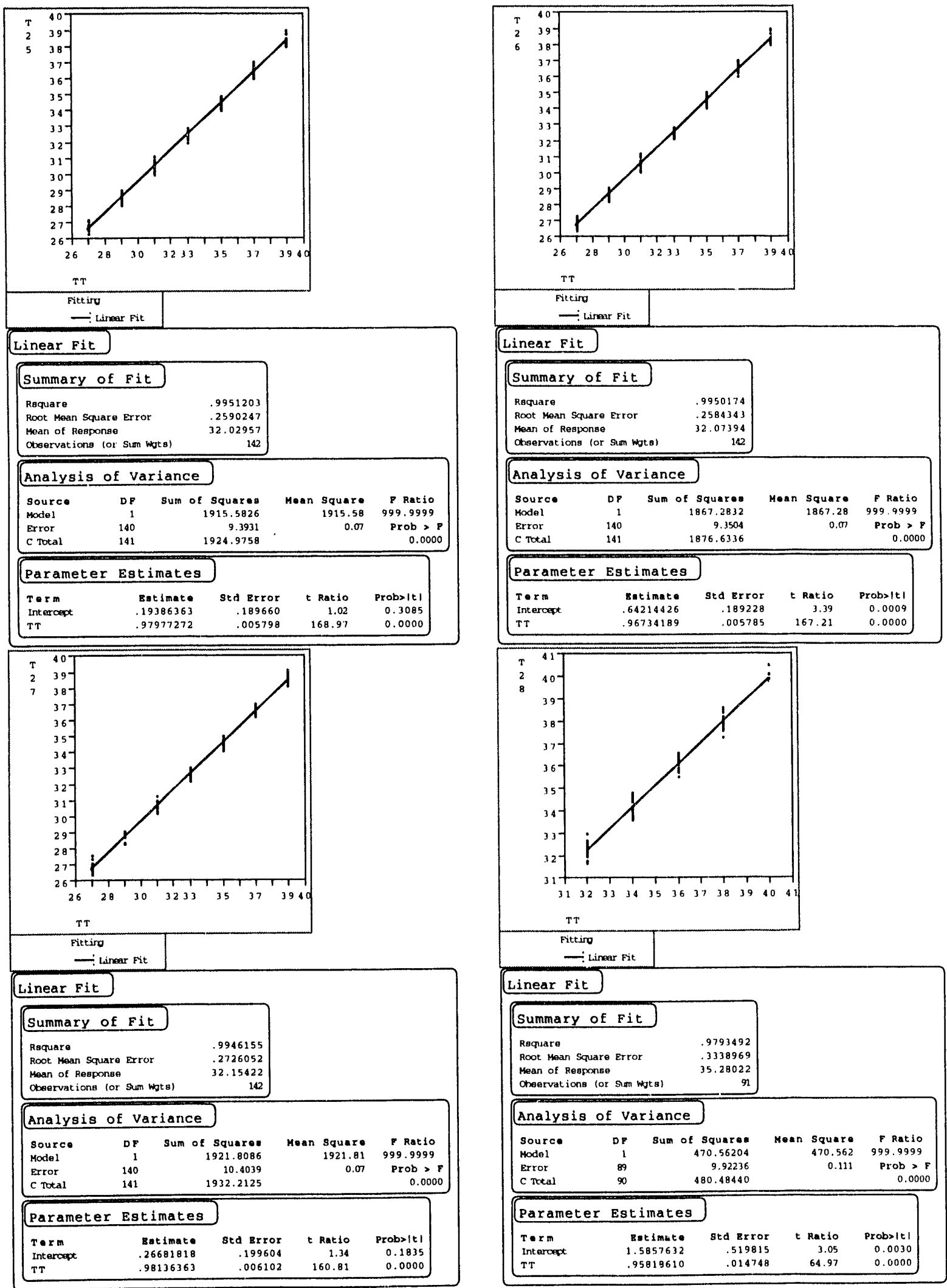


\section{Acclimation Experiment Tanks 29-32}
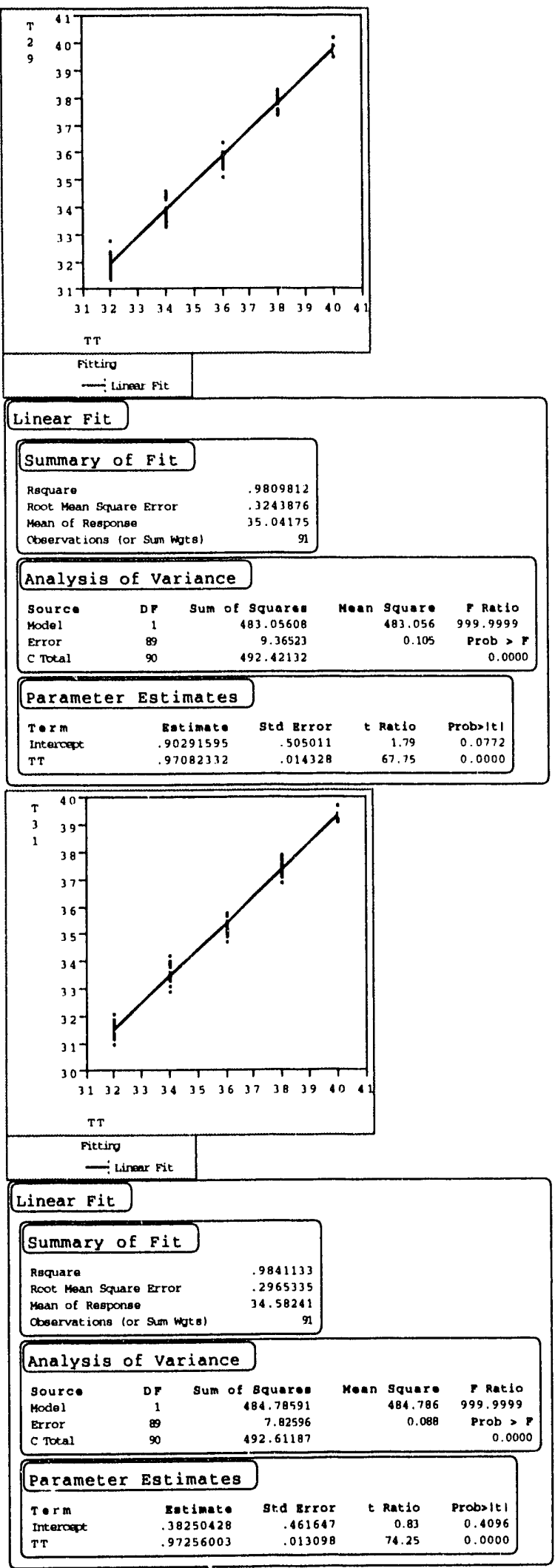
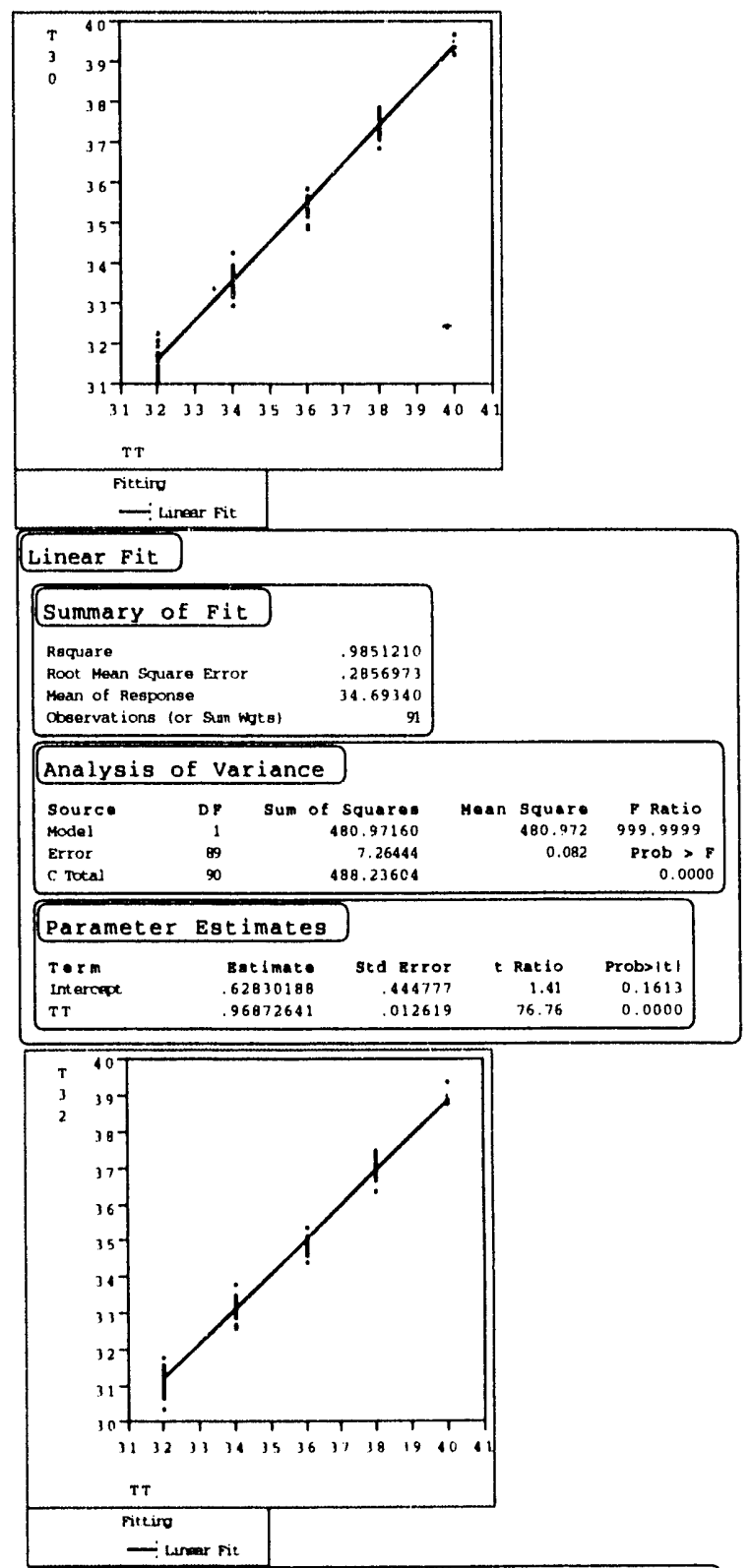

Linear Fit

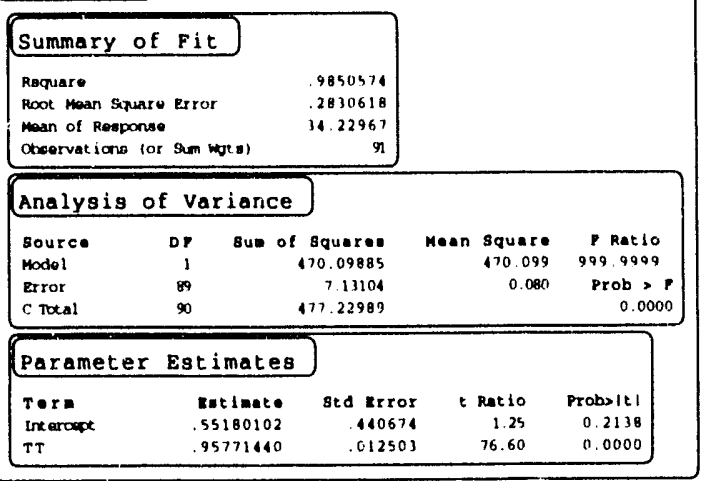




\section{Acclimation Experiment Tanks 33-36}
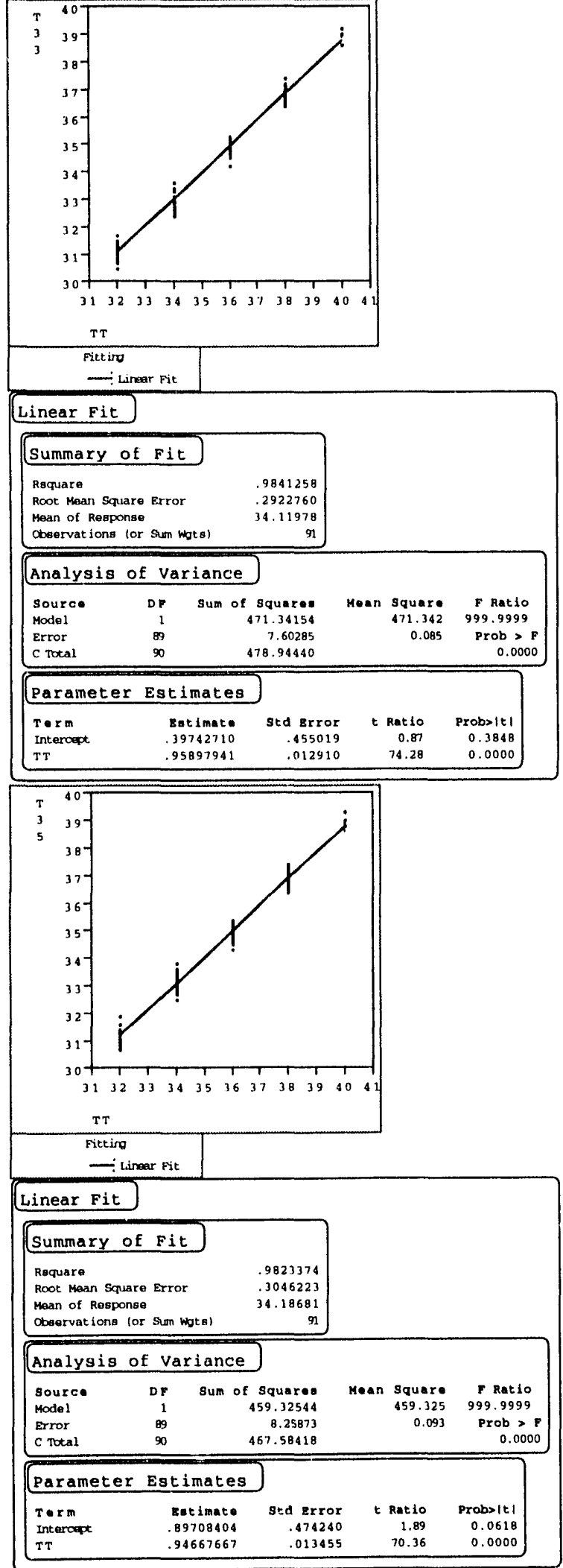
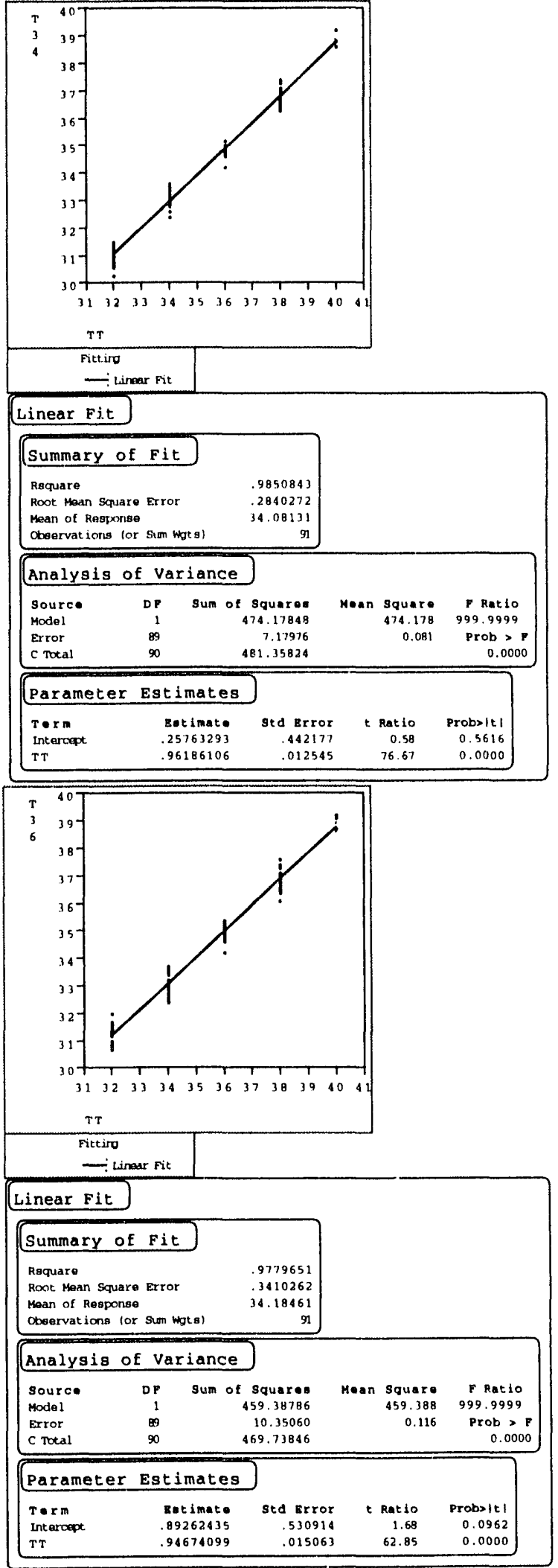


\section{Rate Experiment Tanks 10-13}
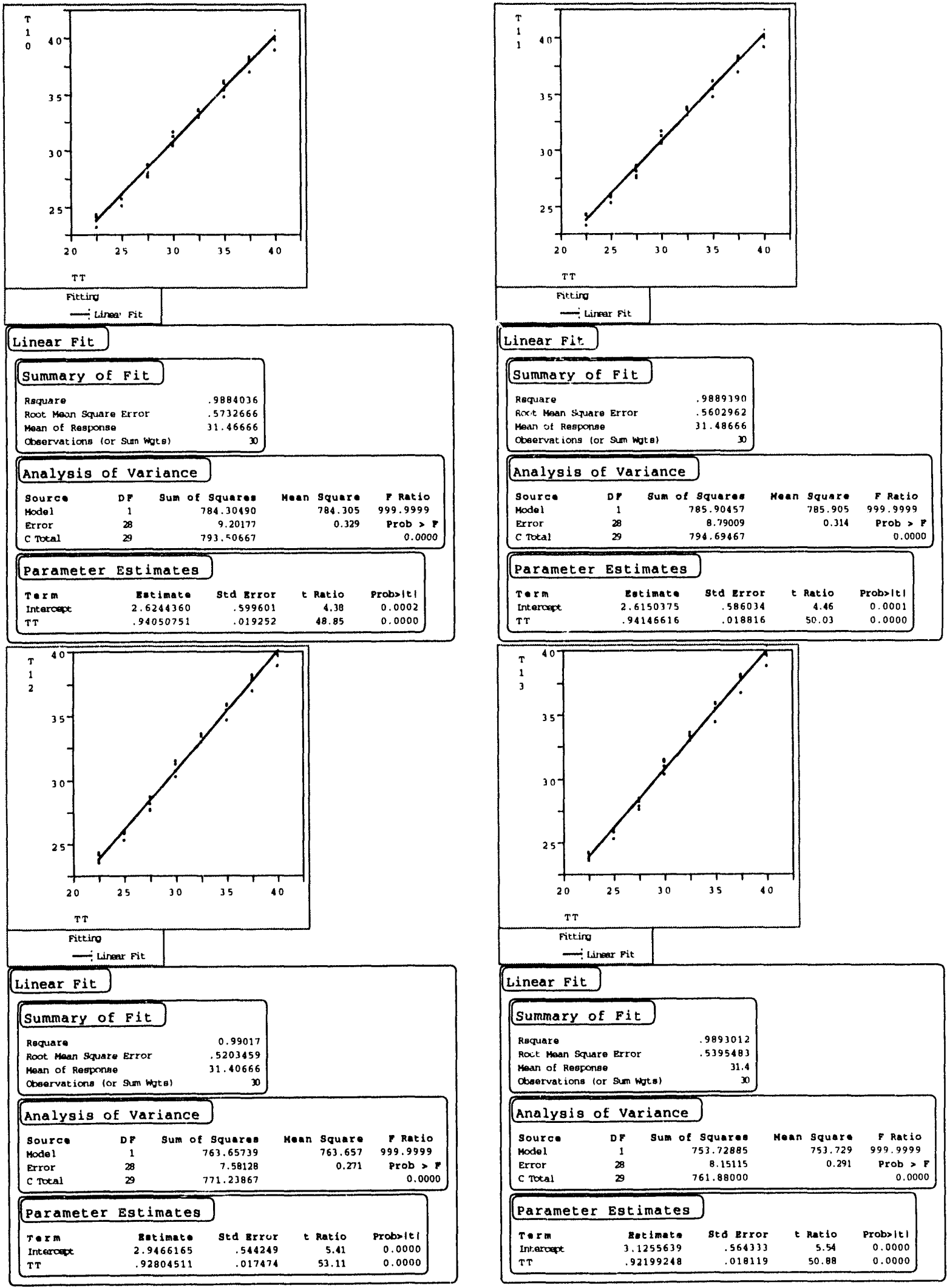


\section{Rate Experiment Tanks 14-17}
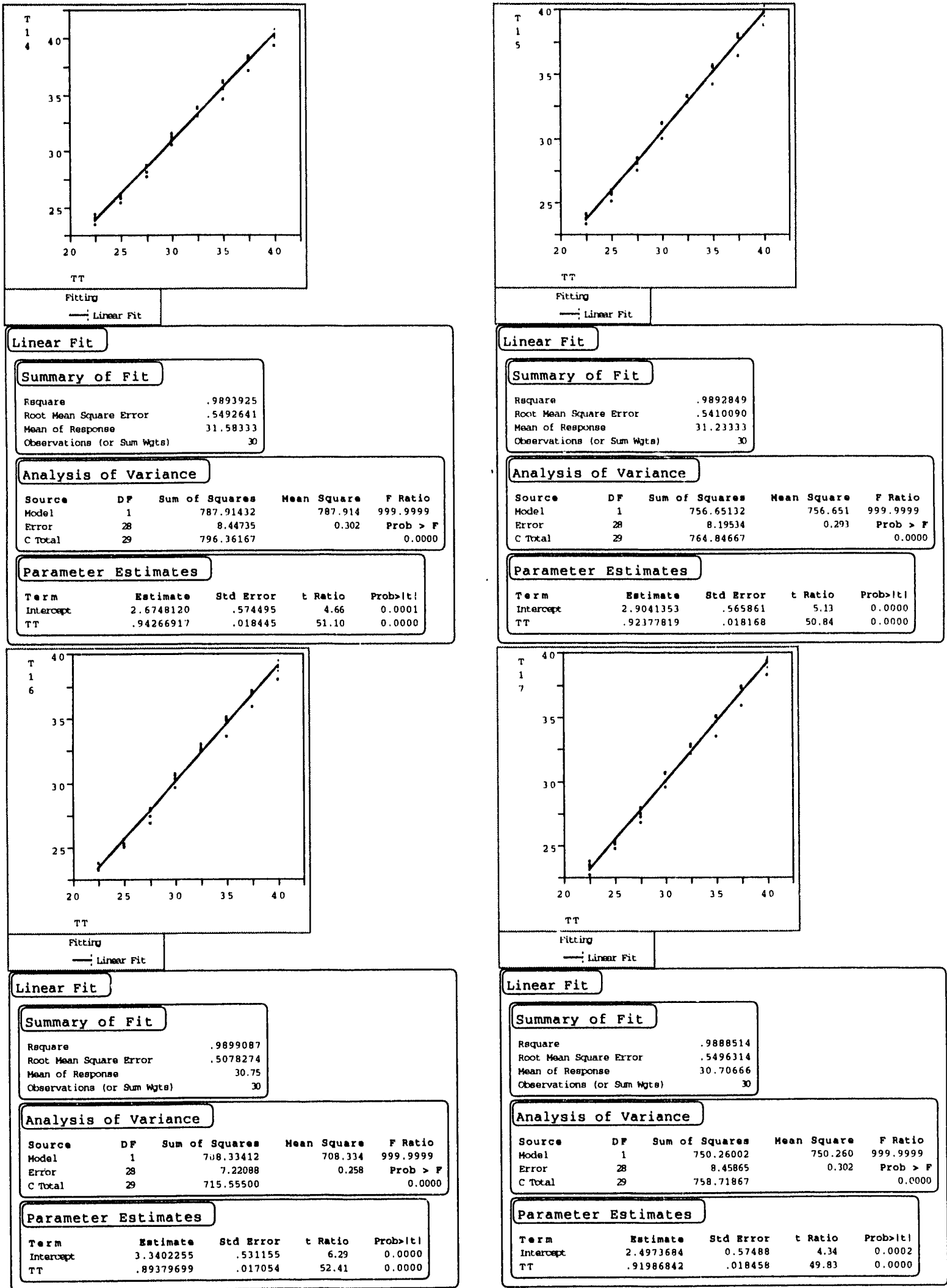


\section{Rate Experiment Tanks 18-21}
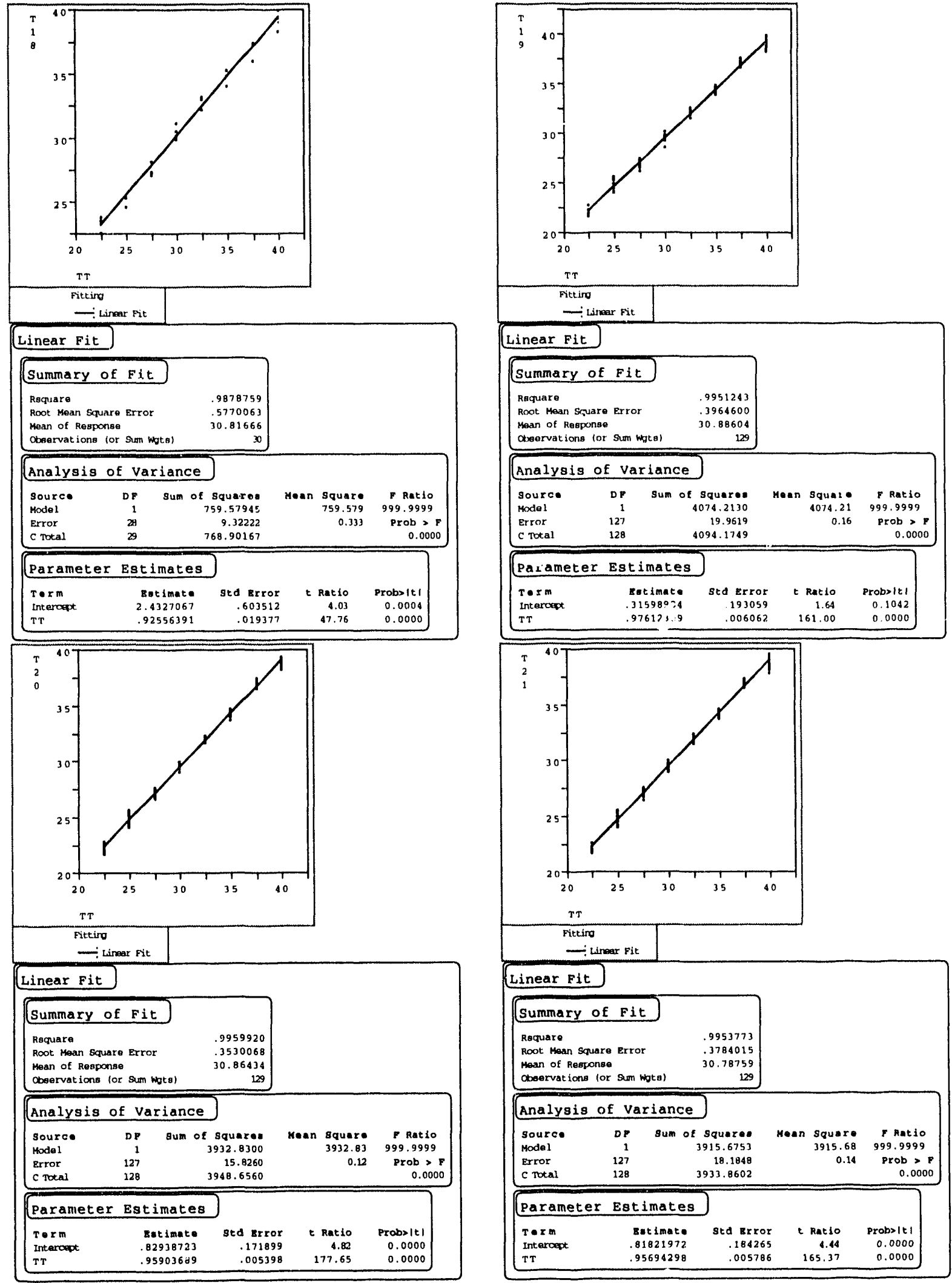
smallmouth bass, Micropterus dolomieui Lacepede", J. Fish Biol., 35:765-780

\section{References}

Brown, H. W., 1974, "Handbook on the effects of temperature on some North American fishes", American Electric Power Service Corporation, Canton, $\mathrm{OH}$.

Bryan, J. D., S. W. Kelsch, and W. H. Neill, 1990, "The Maximum Power Principle in Behavioral Ther-
U. S. Department of Energy, 1990, "Final Environmental Impact Statement" Continued Operation of $\mathrm{K}-, \mathrm{L}-$, and P-Reactors, Savannah River Site, Aiken, South Carolina", DOE/EIS-0147

Cox, D. R. and D. Oakes, 1984, Analysis of Survival Data. Chapman and Hall, London.

\section{Rate Experiment Tanks 22-25}
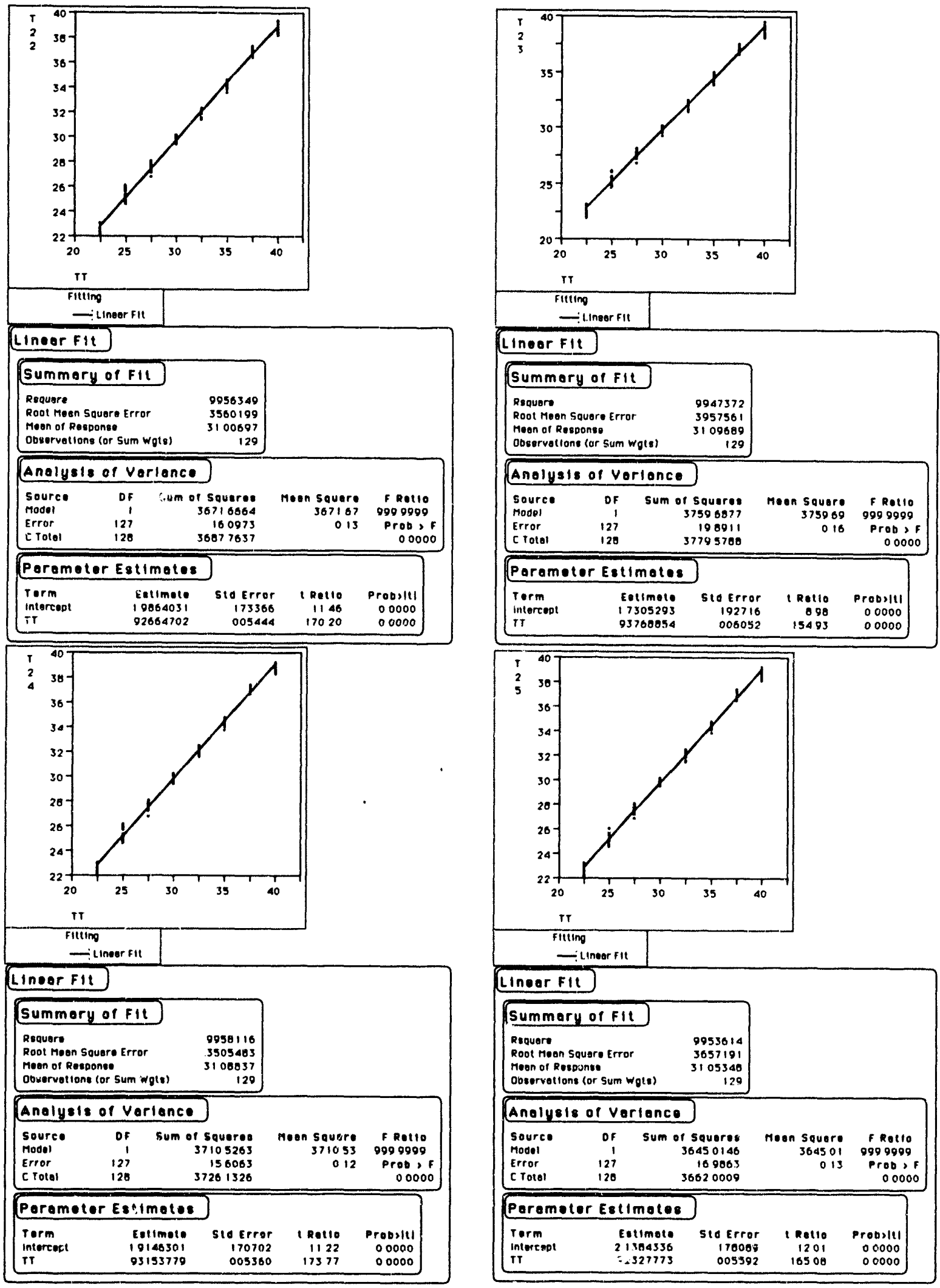


\section{Rate Experiment Tanks 26-29}
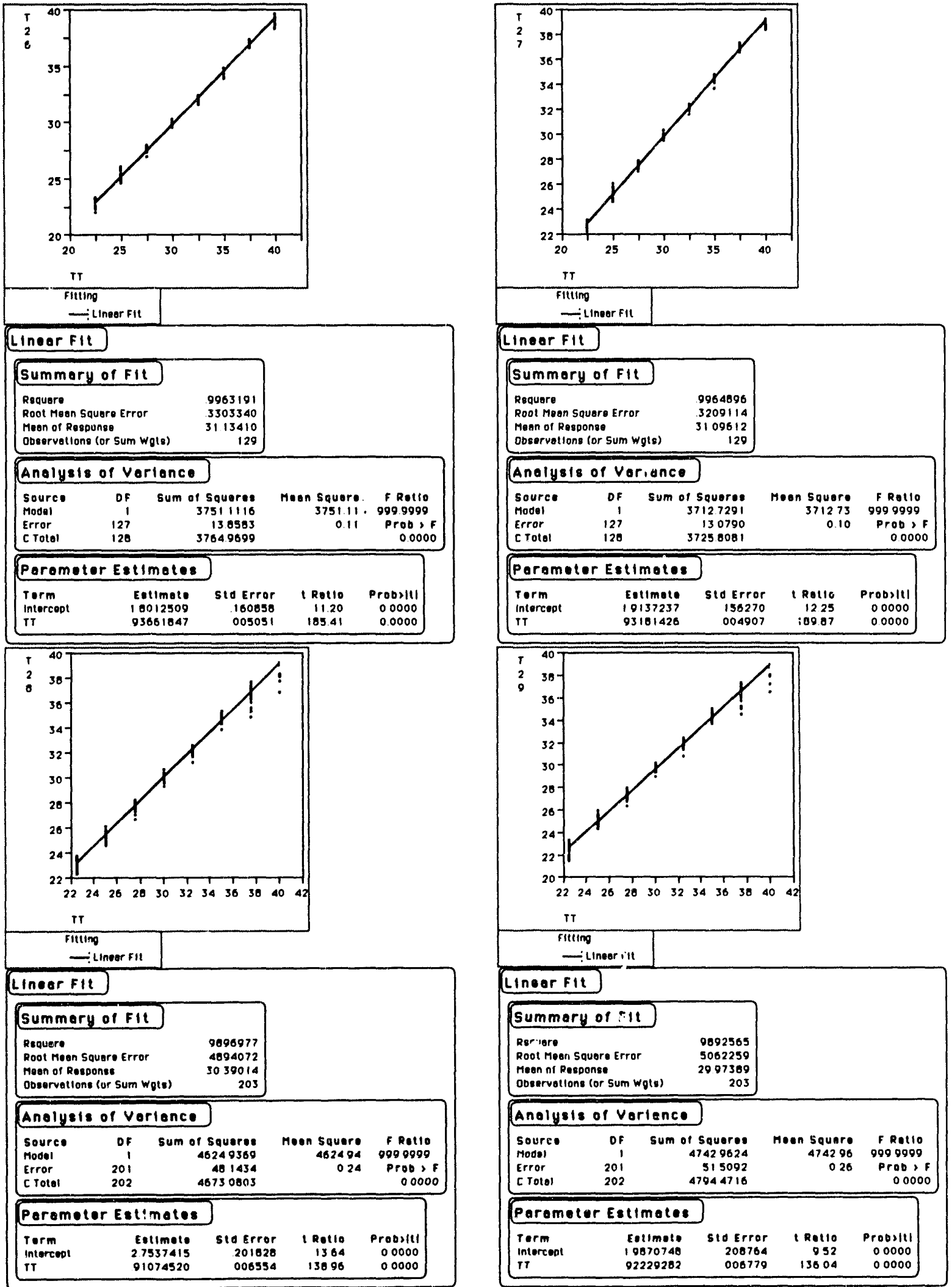


\section{Rate Experiment Tanks 30-33}
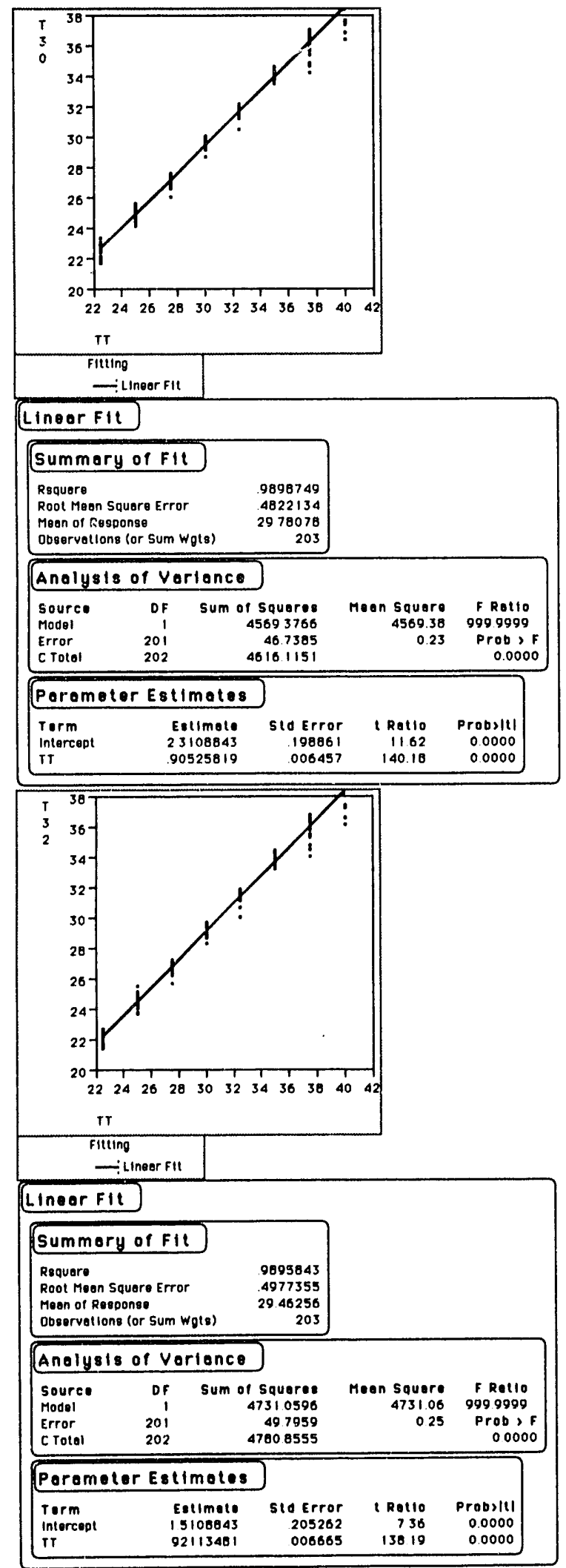
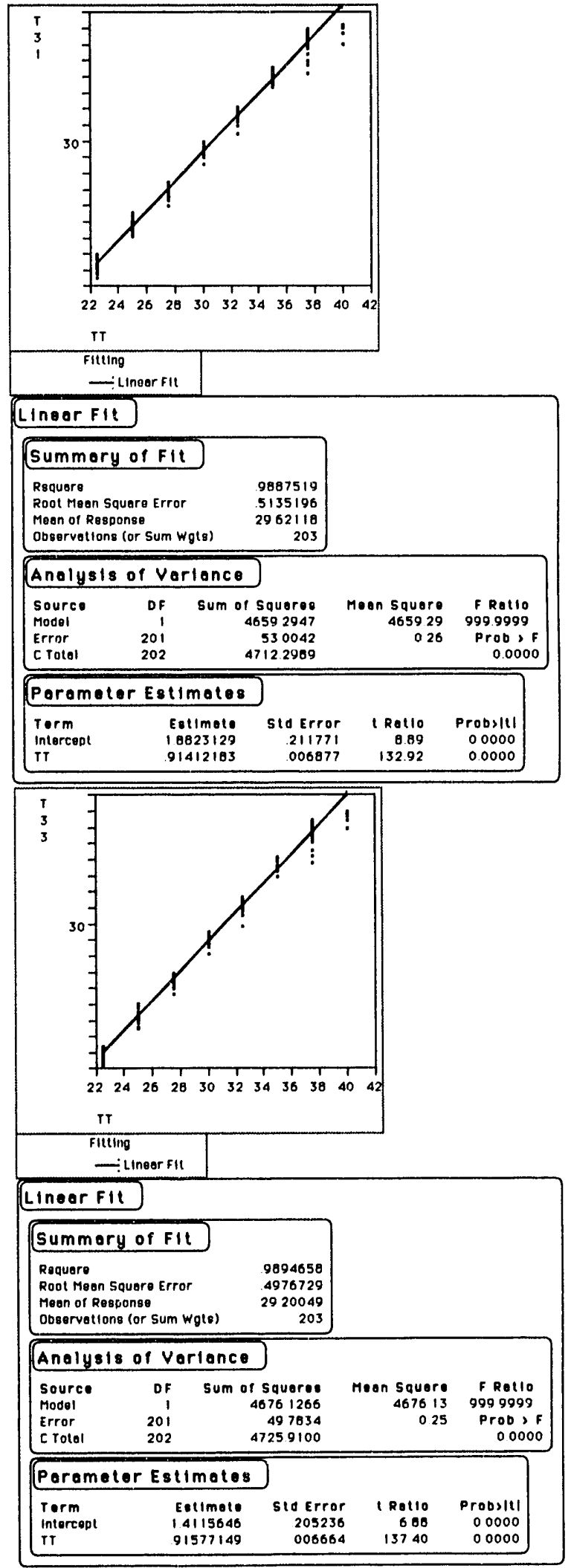


\section{Rate Experiment Tanks 34-36}
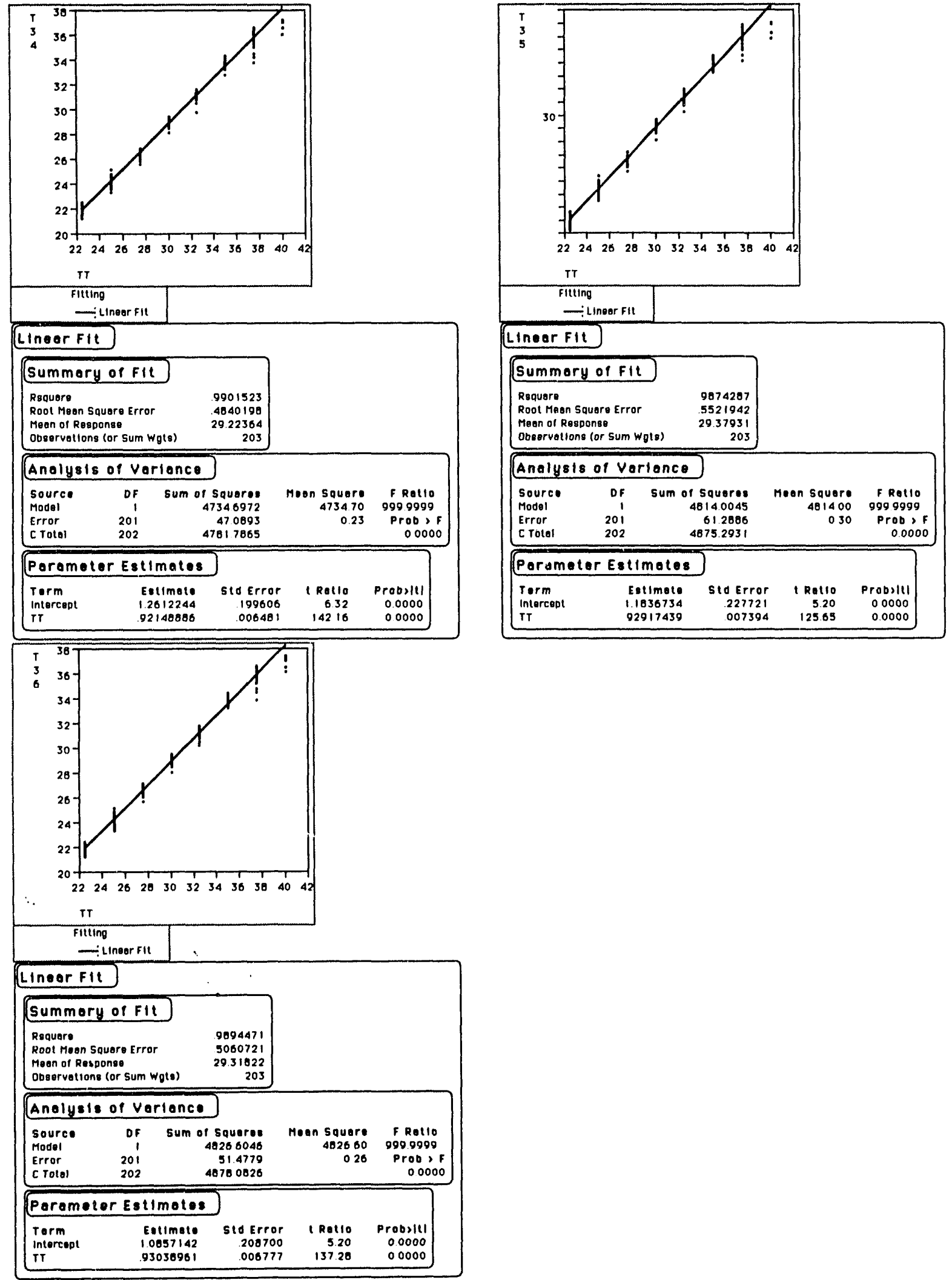


\section{Appendix B}




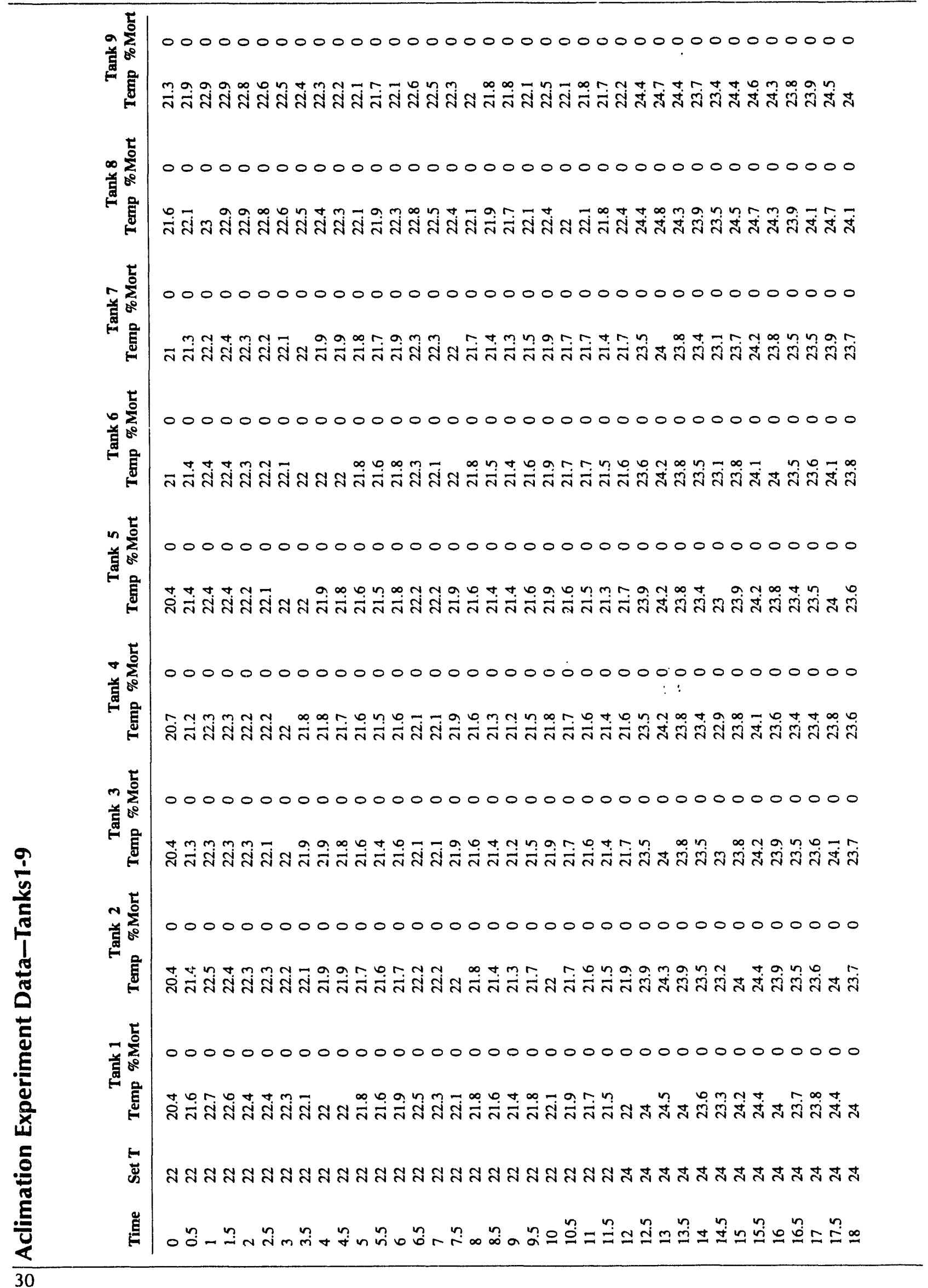




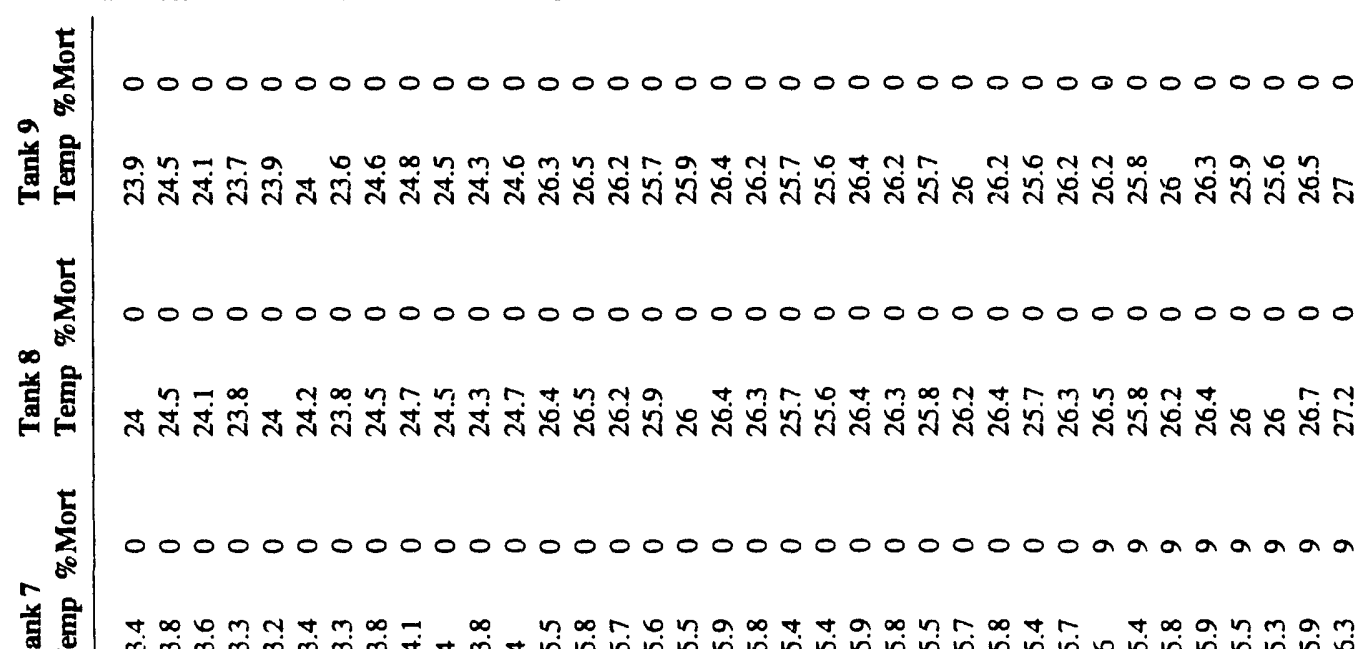

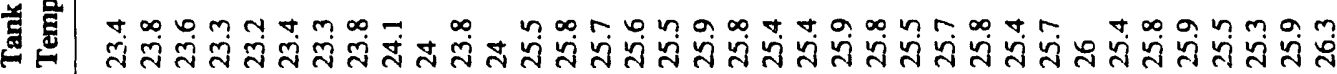

000000000000000000000000000000000000

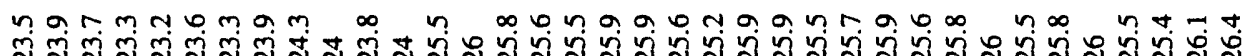

000000000000000000000000000000000000

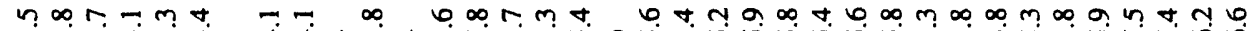

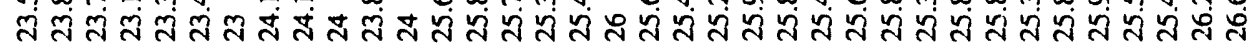

000000000000000000000000000000000000 $+8$

t.

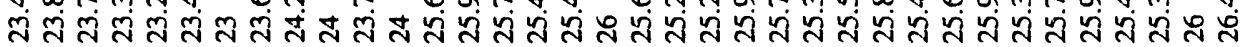
000000000000000000000000000000000000

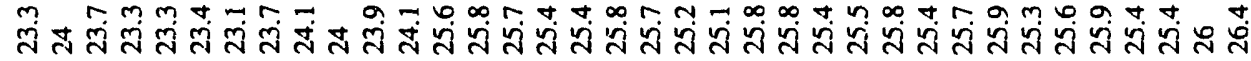
000000000000000000000000000000000000

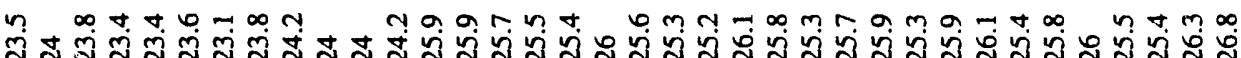

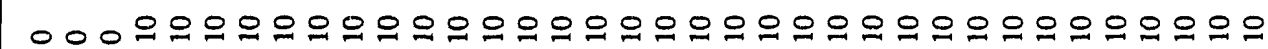

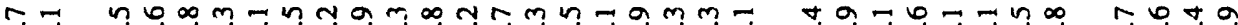

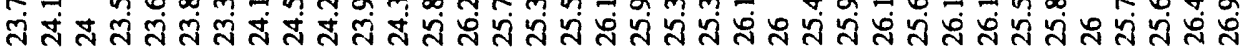

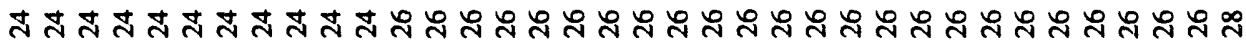


屍

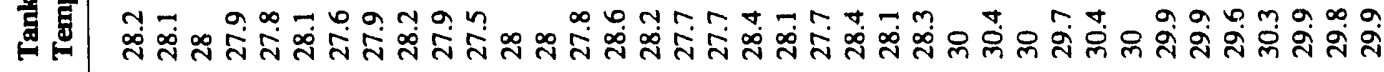

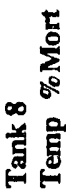

0000000000000000000000000000000000000

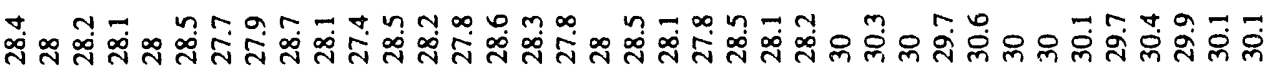

a a a a a a a a a a a a a a a a a a a a a a a a a a a a a a a a

b o n

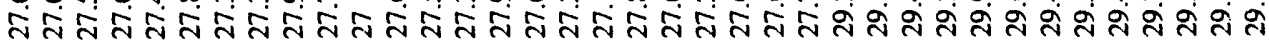

0000000000000000000000000000000000000

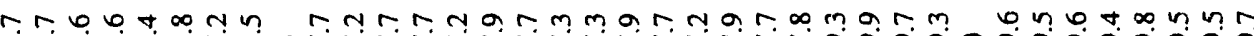
जัন

0000000000000000000000000000000000000

r. ন

0000000000000000000000000000000000000

m n ন

0000000000000000000000000000000000000

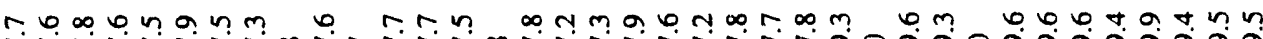
ন

0000000000000000000000000000000000000

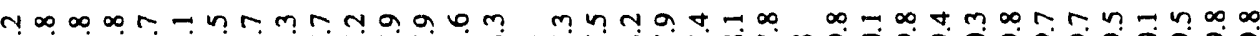
๙ ते

으으으으으이으으으으으으으으으으응으으으으으으으으으으으으으으으으으으 4 $\infty$ o

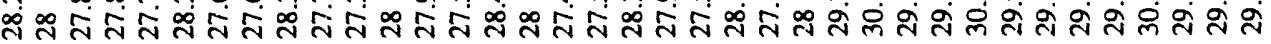

ని జి

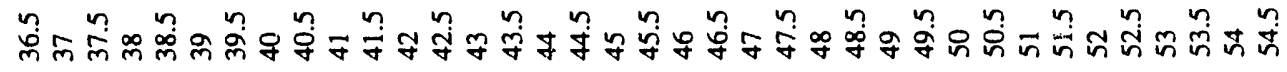


으으응ㅇㅇㅇㅇㅇㅇㅇㅇㅇㅇㅇㅇㅇㅇㅇㅇㅇㅇㅇㅇㅇ으으으으으응ㅇㅇㅇㅇㅇㅇㅇㅇㅇㅇㅇㅇㅇㅇㅇㅇㅇ

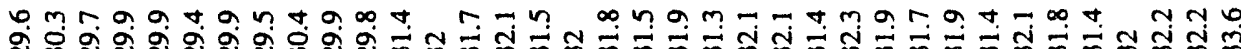

㮍

000000000000000000000000000000000000

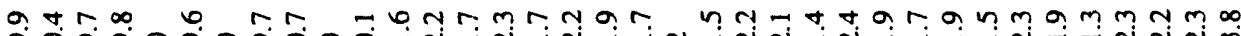

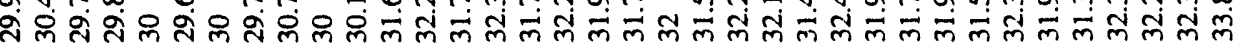

a a a a a a a a a a a a a a a a a a a a a a a a a a a a a a a a

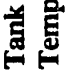

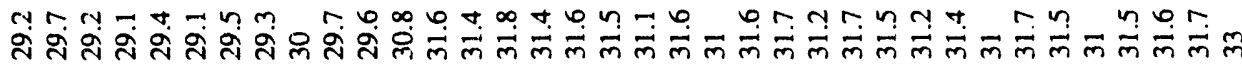

000000000000000000000000000000000000

momm m n t-

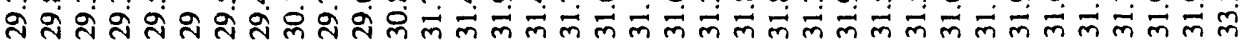

000000000000000000000000000000000000

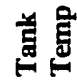

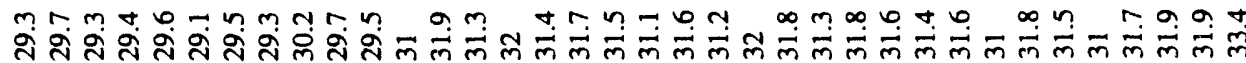

000000000000000000000000000000000000

$+8$

弟 冒

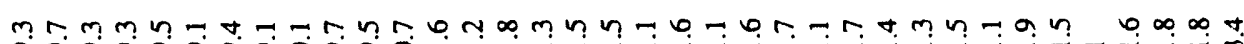

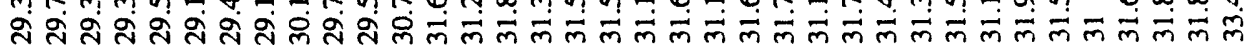

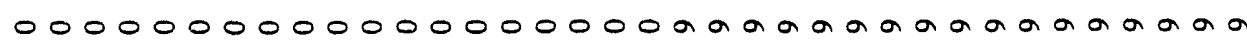

mr.t.

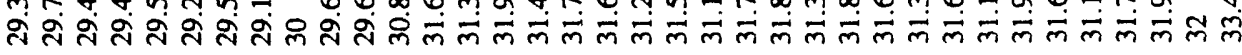

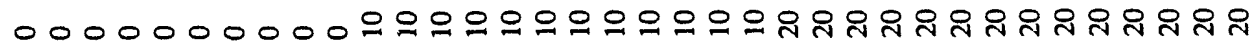
N

วิ유

으으응ㅇㅇㅇㅇㅇㅇㅇㅇㅇㅇㅇㅇㅇㅇㅇㅇㅇㅇㅇㅇ으으으으으으으으으으으으응ㅇㅇㅇㅇㅇㅇㅇㅇㅇ

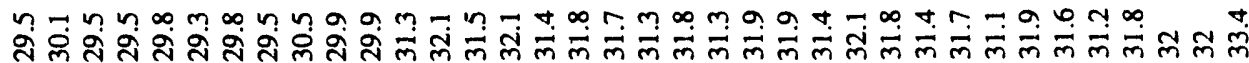

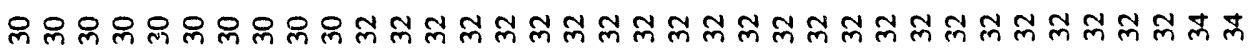

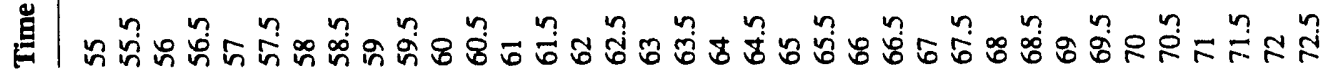




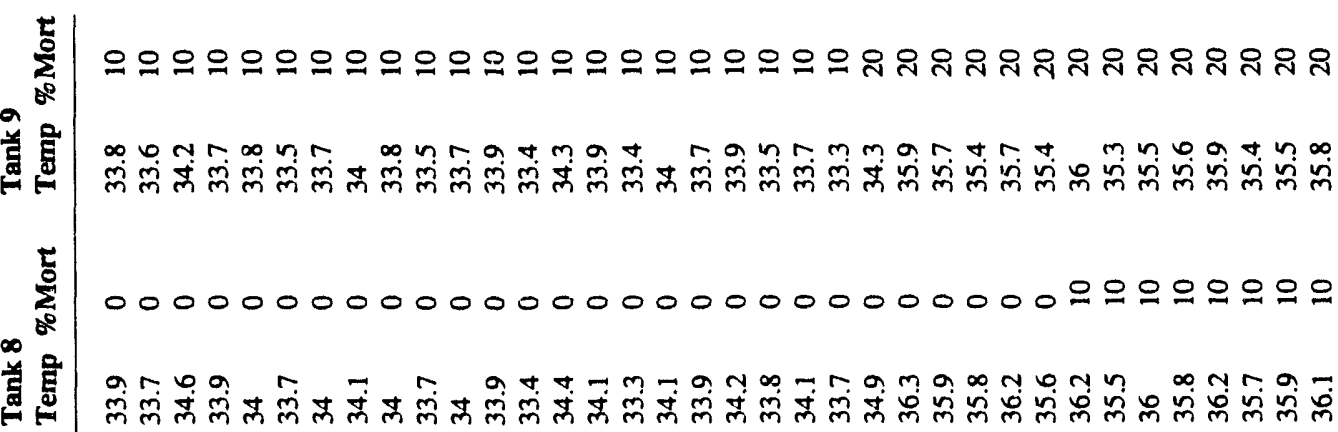

a a a a a a a a a a a a a a a a a a a a a a a a a a a a

恶总

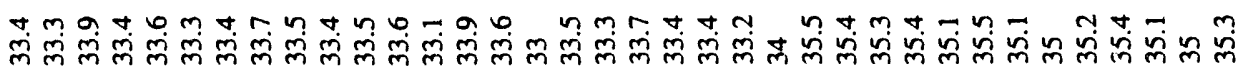

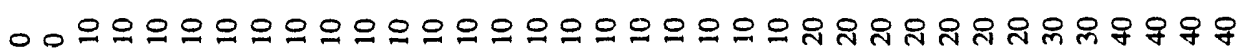

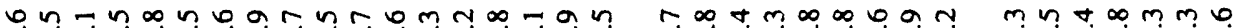

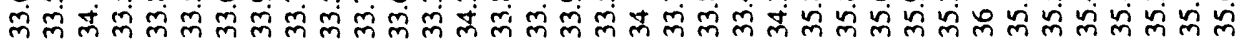

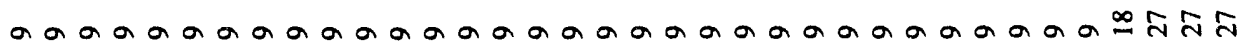

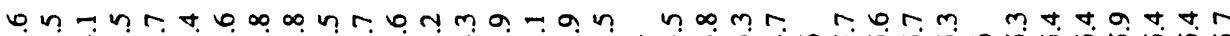

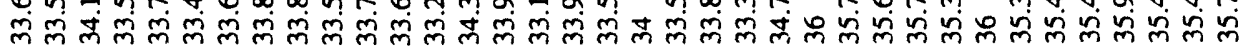

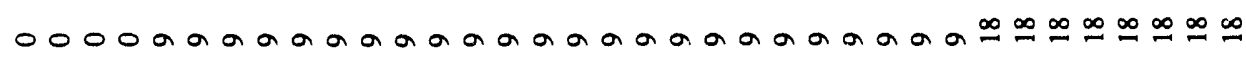

$\nabla$

总是

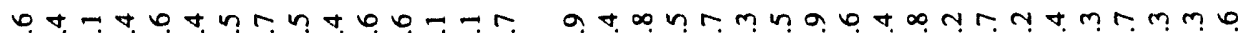

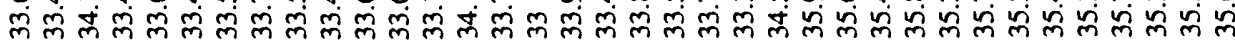

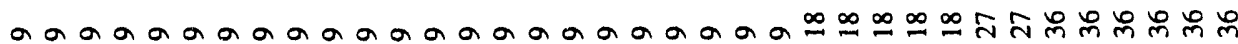
$m$

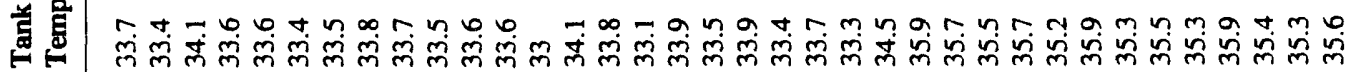

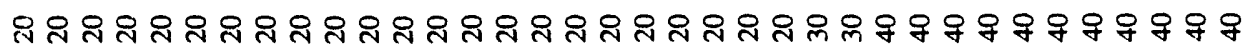

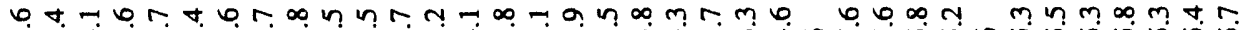
लं लं

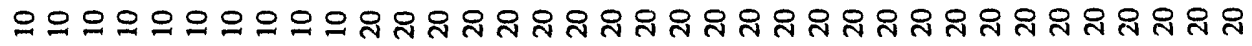

rnNo on

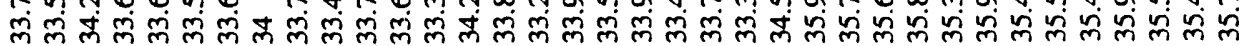

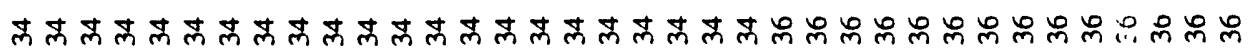

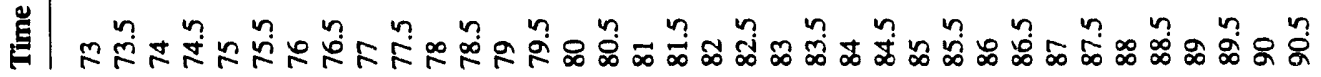


象

藏苞

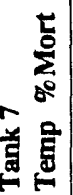

总苞

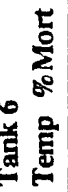

的总

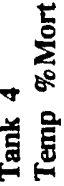

$\sum_{0}^{5}$

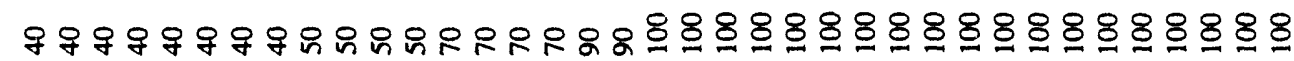

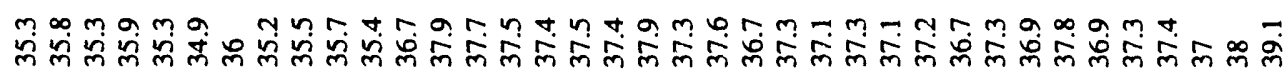

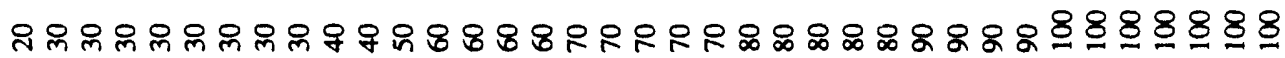

$m \infty+\infty$ t

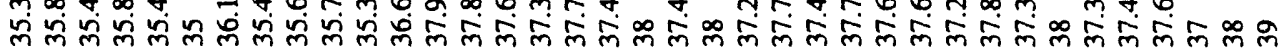

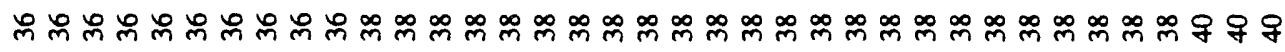

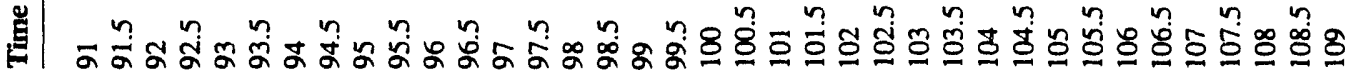


00000000000000000000000000000000000000

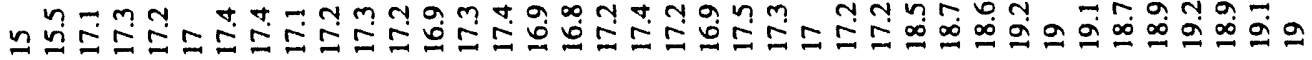

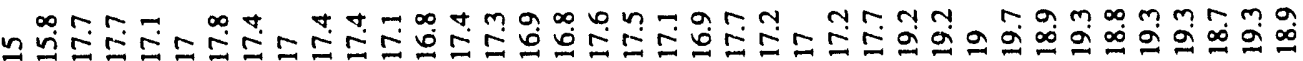
r

00000000000000000000000000000000000000

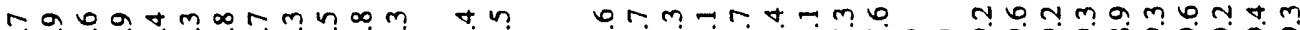

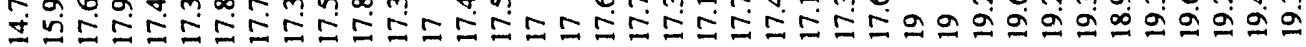

00000000000000000000000000000000000000

๓

00000000000000000000000000000000000000

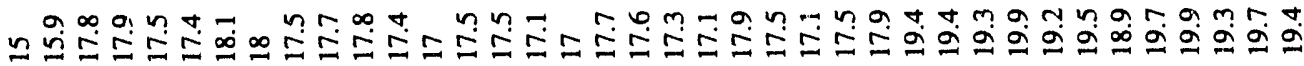

00000000000000000000000000000000000000 r.

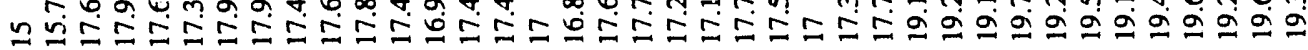
00000000000000000000000000000000000000 N உ

m NaOn 0 Thrt-mo

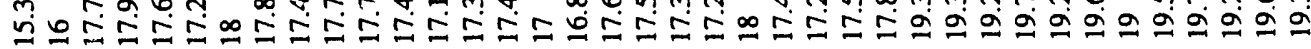

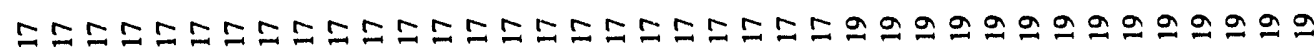


000000000000000000000000000000000000

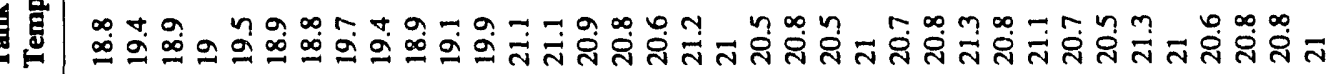

000000000000000000000000000000000000

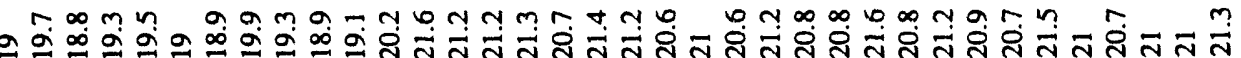

000000000000000000000000000000000000

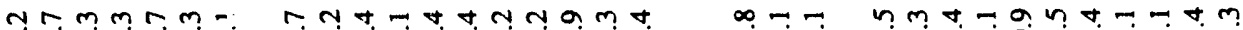

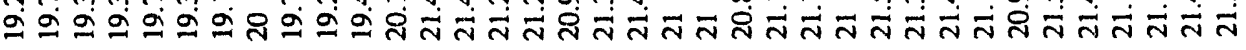

000000000000000000000000000000000000

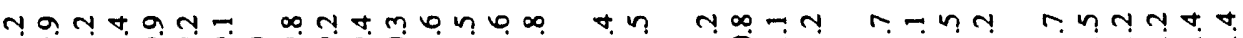

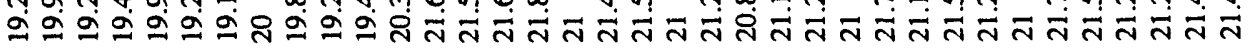

000000000000000000000000000000000000 in

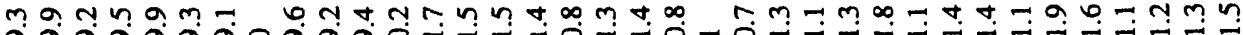

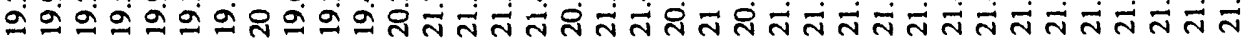

000000000000000000000000000000000000 $+8$

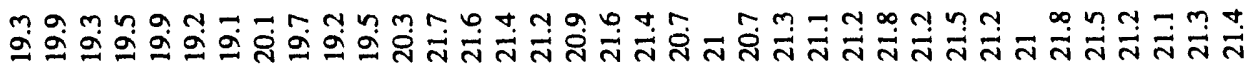

000000000000000000000000000000000000

4r. פั่ 000000000000000000000000000000000000 N

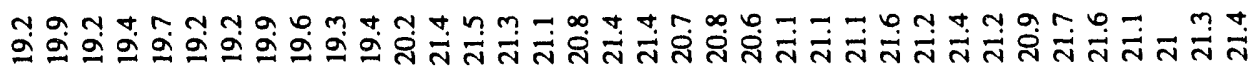
000000000000000000000000000000000000

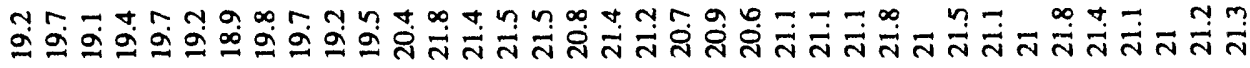

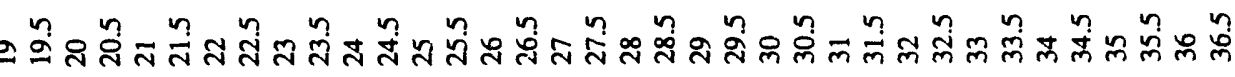




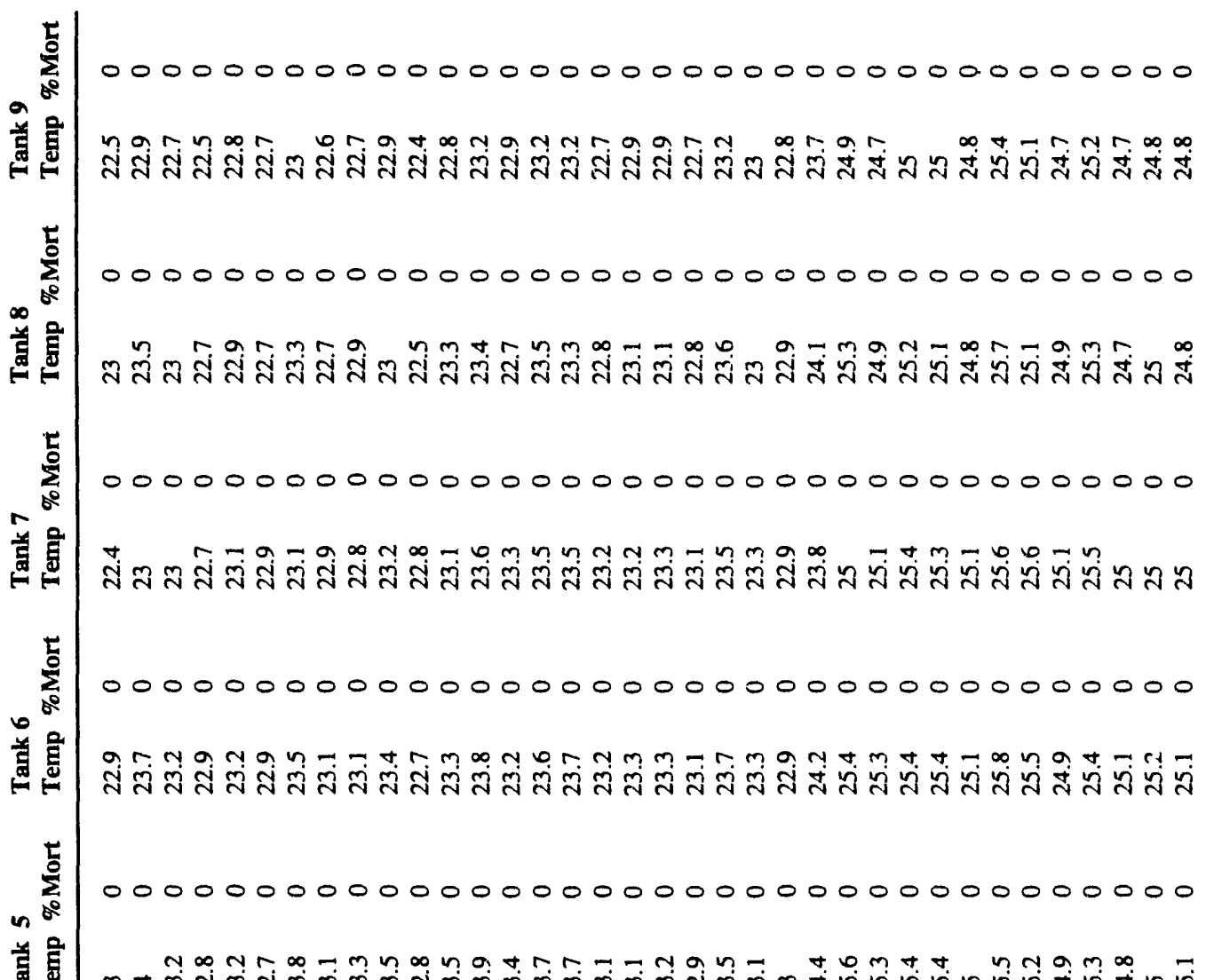

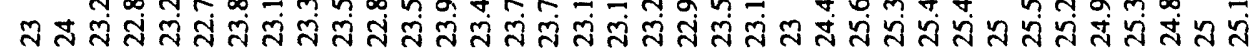

000000000000000000000000000000000000

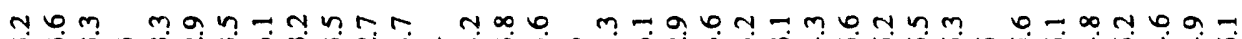

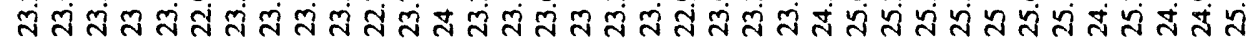

000000000000000000000000000000000000 $a n-a=0$ ด

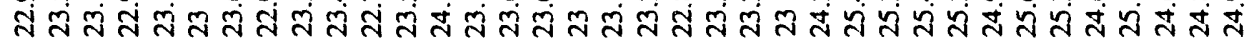
000000000000000000000000000000000000

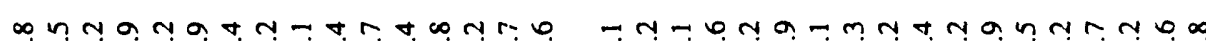

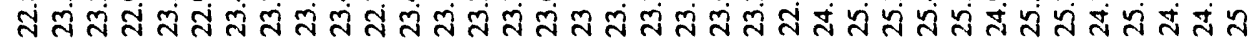
000000000000000000000000000000000000 ๗

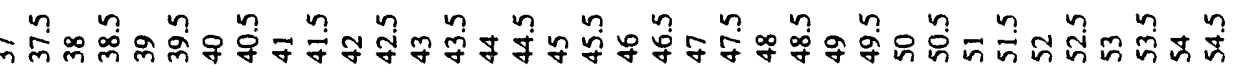




\begin{tabular}{|c|c|c|c|c|c|c|c|c|c|c|c|}
\hline \multirow{2}{*}{\multicolumn{6}{|c|}{ Analysis of Varlance }} & \multicolumn{6}{|c|}{ 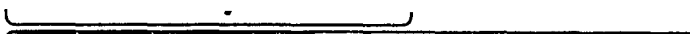 } \\
\hline & & & & & & \multicolumn{6}{|c|}{ Analysls of Vartance } \\
\hline $\begin{array}{l}\text { Source } \\
\text { Modol } \\
\text { Error } \\
\text { c rotel }\end{array}$ & $\begin{array}{r}\text { OF } \\
1 \\
127 \\
128\end{array}$ & sum of & $\begin{array}{l}\text { Soueres: } \\
7105263 \\
156083 \\
7261326\end{array}$ & $\begin{array}{r}\text { moen sauere } \\
371053 \\
012\end{array}$ & $\begin{array}{r}\text { F Rell10 } \\
99999990 \\
\text { Prob if } \\
00000\end{array}$ & $\begin{array}{l}\text { Sourco } \\
\text { Model } \\
\text { Error } \\
\text { C Total }\end{array}$ & $\begin{array}{l}\text { of } \\
1 \\
127 \\
120\end{array}$ & sum of & $\begin{array}{l}\text { Squeros } \\
6450146 \\
169863 \\
6620009\end{array}$ & $\begin{array}{r}\text { Meon Squer: } \\
3645 \text { o } \\
013\end{array}$ & $\begin{array}{r}\text { FRo110 } \\
99999909 \\
\text { Prob }, ~ \\
00000\end{array}$ \\
\hline \multicolumn{6}{|c|}{ Porometer Esilmotes } & \multicolumn{6}{|c|}{ Poramoter Estimotos } \\
\hline $\begin{array}{l}\text { Term } \\
\text { intercopt } \\
\text { TT }\end{array}$ & : & $\begin{array}{l}1110010 \\
116301 \\
153770\end{array}$ & $\begin{array}{r}\text { S10 Error } \\
170702 \\
005380\end{array}$ & $\begin{array}{r}\text { IRet110 } \\
1122 \\
17377\end{array}$ & $\begin{array}{c}\text { Probsit1 } \\
00000 \\
00000\end{array}$ & $\begin{array}{l}\text { Term } \\
\text { intercept } \\
\text { rt }\end{array}$ & $\begin{array}{l}E \\
21 \\
?\end{array}$ & $\begin{array}{l}\text { limete } \\
364336 \\
321773\end{array}$ & $\begin{array}{r}\text { sta Error } \\
178003 \\
005392\end{array}$ & $\begin{array}{l}180110 \\
1201 \\
16508\end{array}$ & $\begin{array}{c}\text { Prob }>111 \\
00000 \\
00000\end{array}$ \\
\hline
\end{tabular}

Response of Fish to Different Simulated Rates of Water Temperature Increase

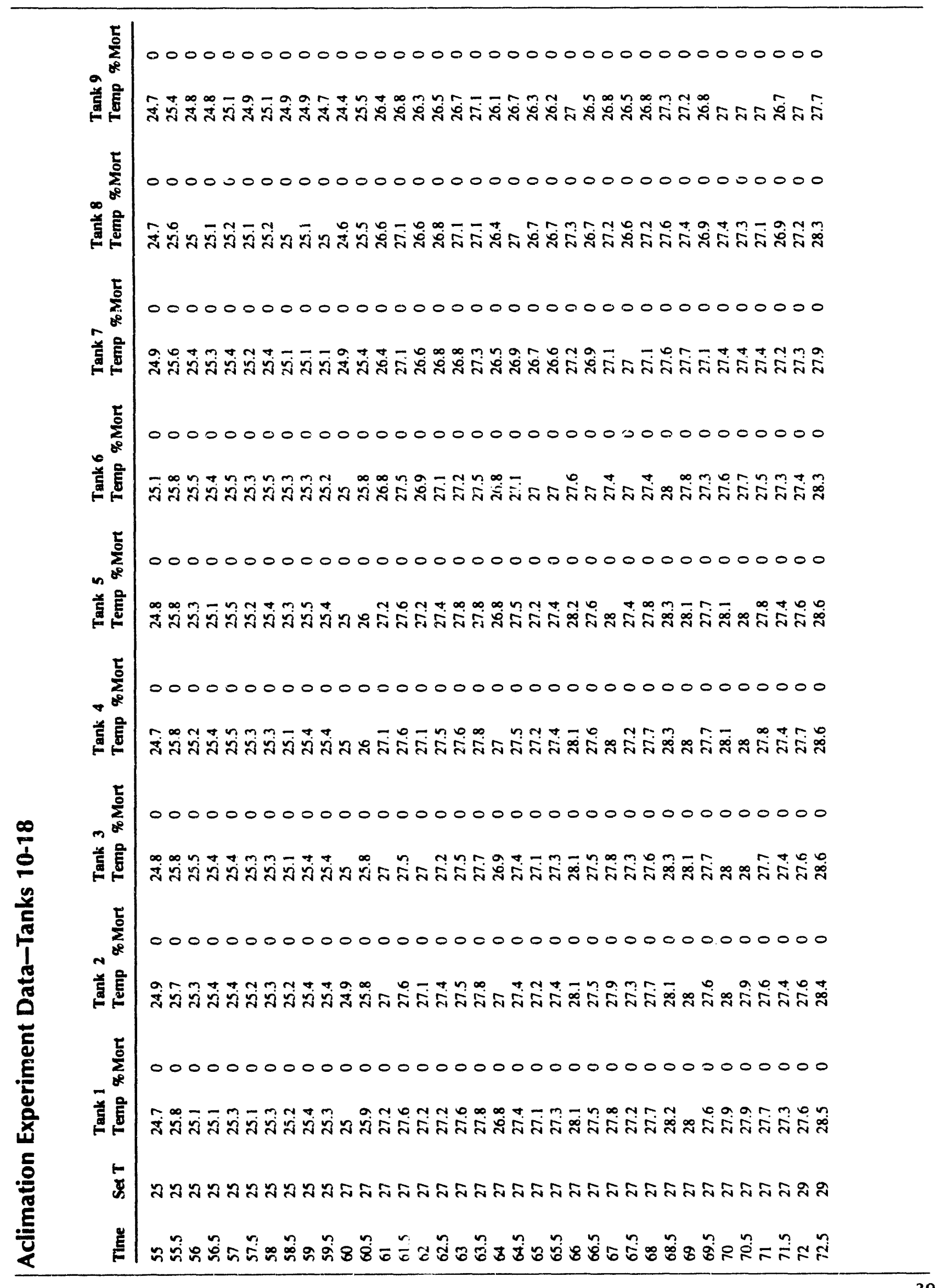




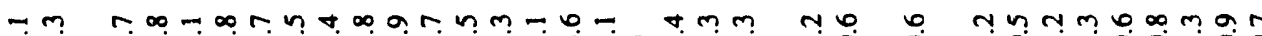

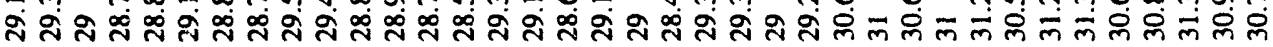

0000000000000000000000000000000000000

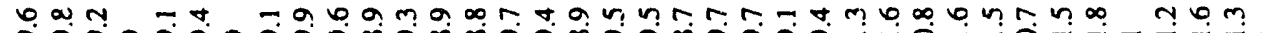

สิ สิ สิ สิ

0000000000000000000000000000000000000

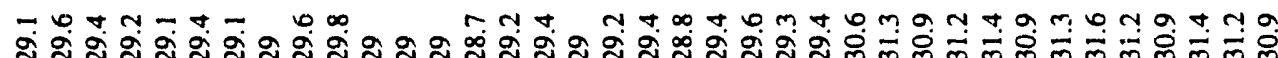

00000000000000000 으으으으으으응ㅇㅇㅇㅇㅇㅇㅇㅇㅇㅇㅡ

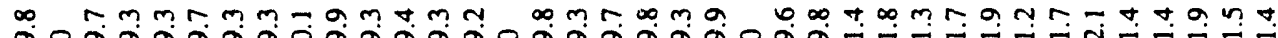

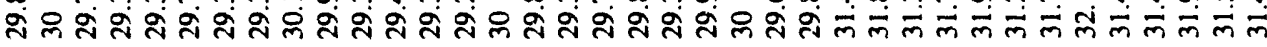

0000000000000000000000000000000000000

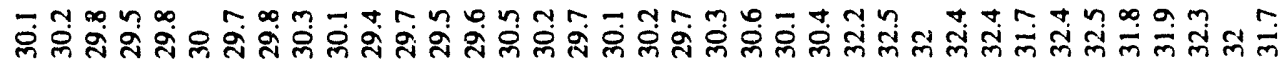

0000000000000000000000000000000000000

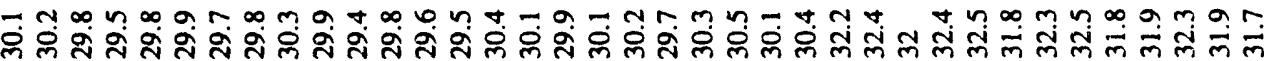

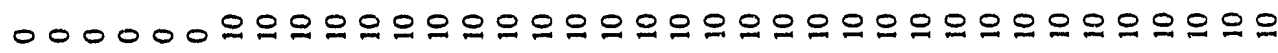

a. ท n

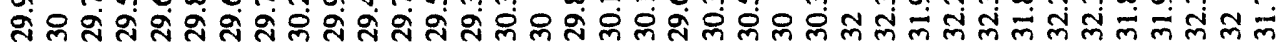
0000000000000000000000000000000000000

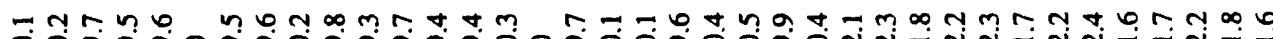

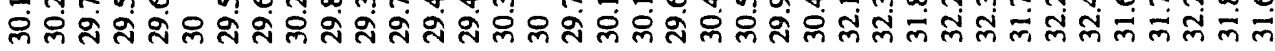

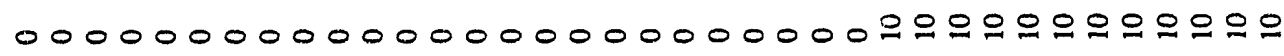

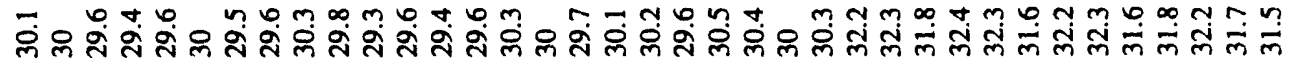

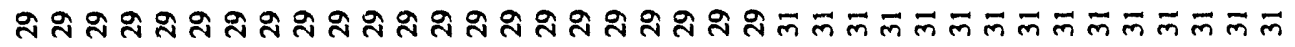

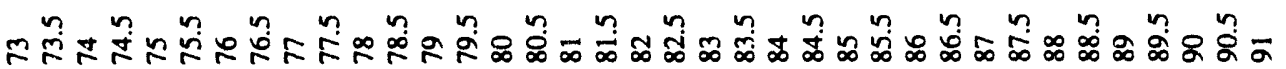




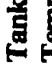

㮍要

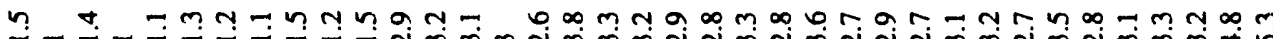

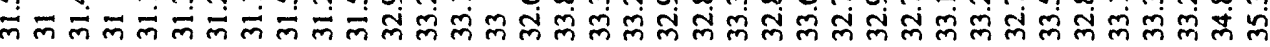

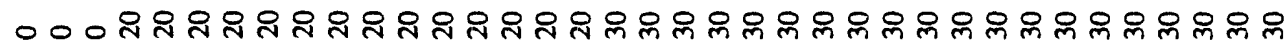

6

总总

的总

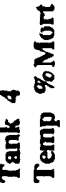

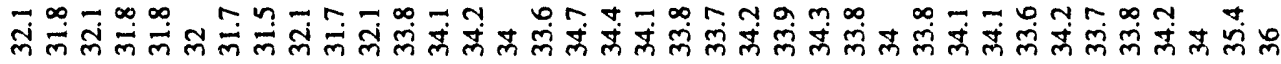

으으으으으으으으으으으으으으으으으으으응ㅇㅇㅇㅇㅇㅇㅇㅇㅇㅇㅇㅇㅇㅇㅇㅇㅇㅇㅇㅇㅇㅇㅇㅇㅡ

$m^{\circ}$

茞号

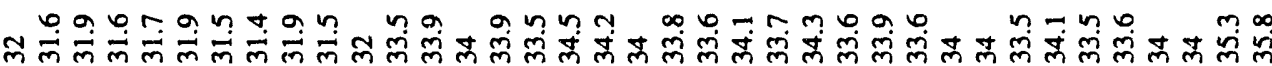

0000000000000000000000000000000000000

$n^{2}$

菟馜

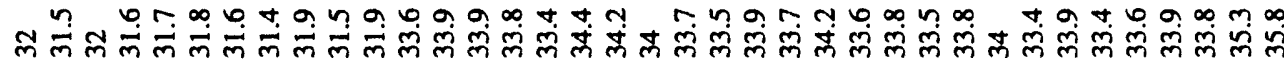

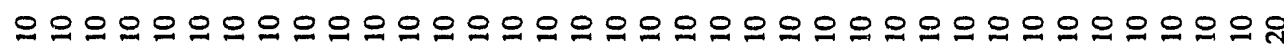

总息

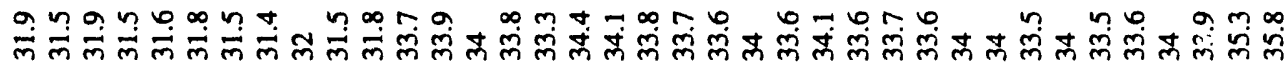

$\ddot{\uplus}$

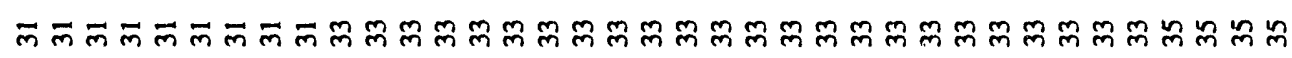

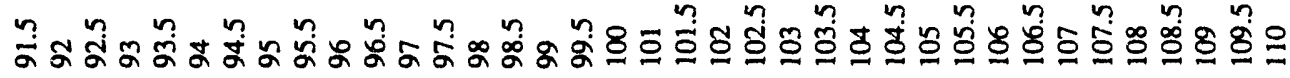




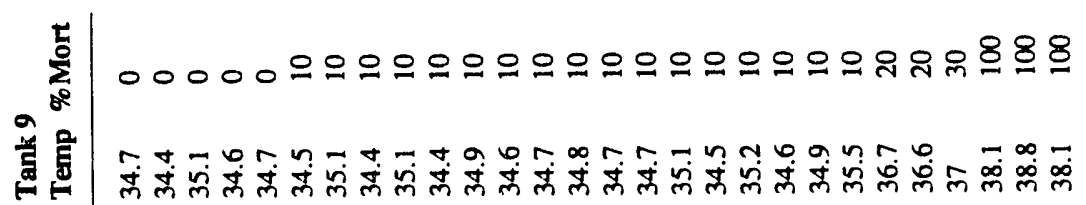

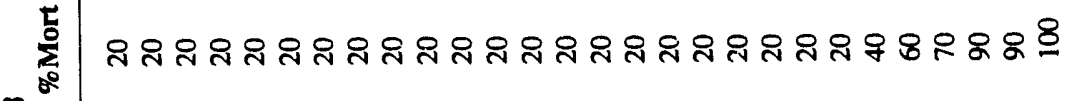

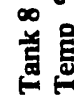

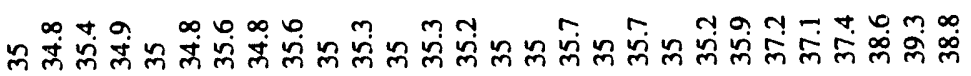

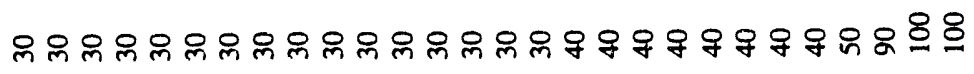

总茞

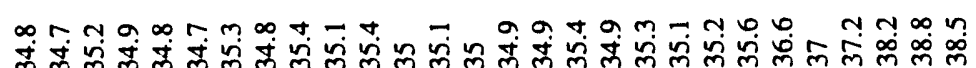

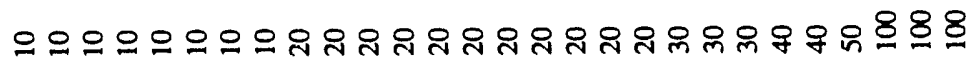

盇苞

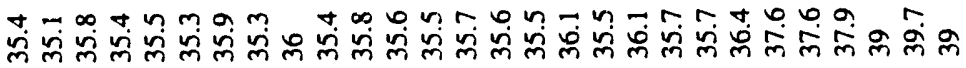

$\sum_{0}^{5}$

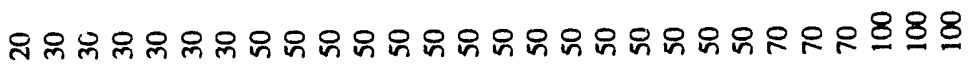

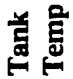

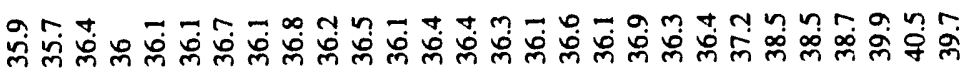

$\sum_{0}^{t}$

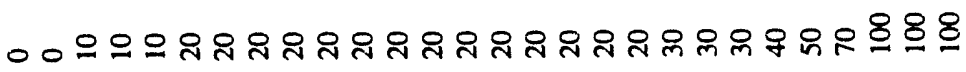

苇 Nonanmam montum-rm totror-no

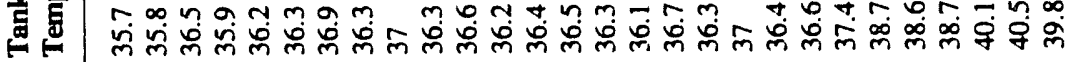

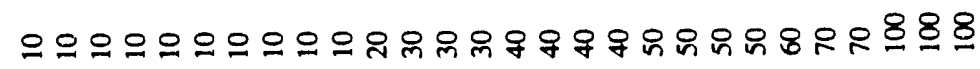

总昢

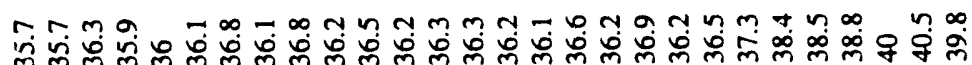

$\sum^{5}$

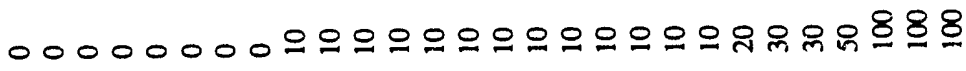

岇魯

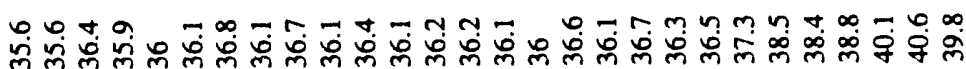

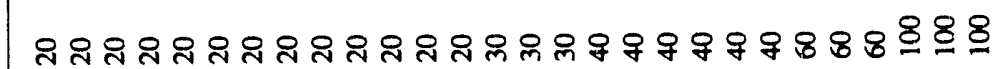

总兽

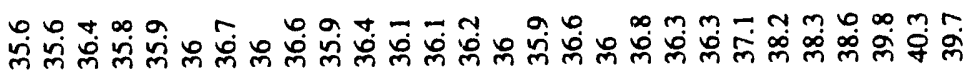

离

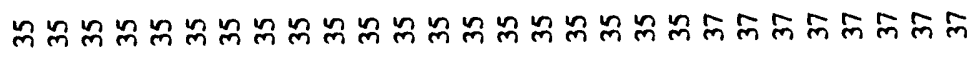

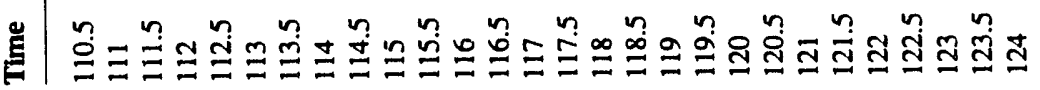


要

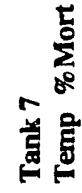

농

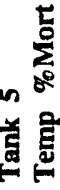

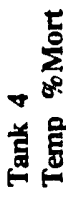

$\sum_{0}^{5}$

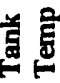

$\sum_{0}^{5}$

曾惫

$\sum_{0}^{5}$

尊亮

\$

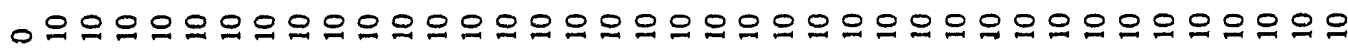

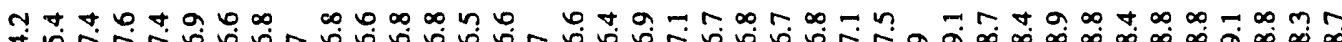

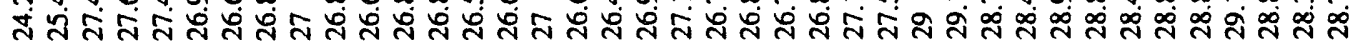

000000000000000000000000000000000000000

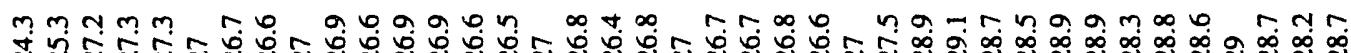

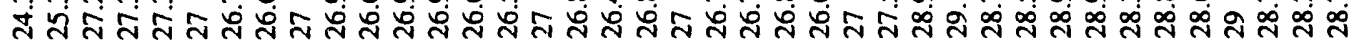

000000000000000000000000000000000000000

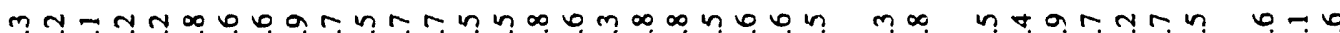

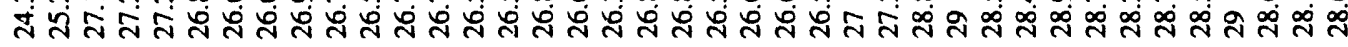

000000000000000000000000000000000000000

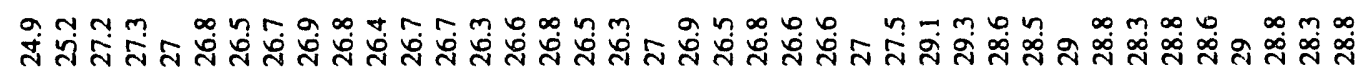

000000000000000000000000000000000000000

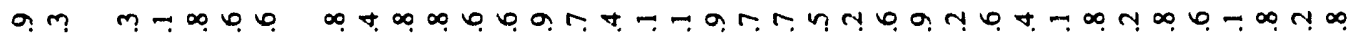

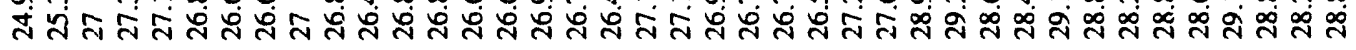

000000000000000000000000000000000000000

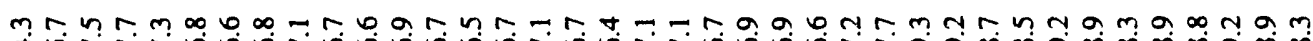

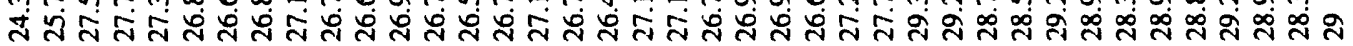

000000000000000000000000000000000000000

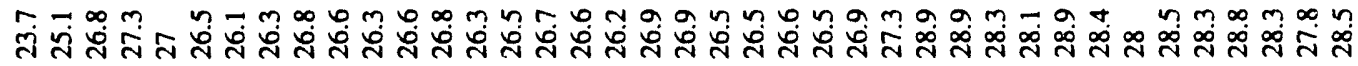

000000000000000000000000000000000000000

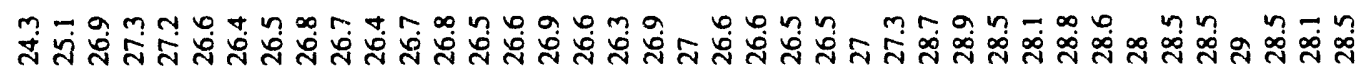

000000000000000000000000000000000000000

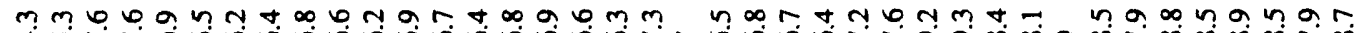

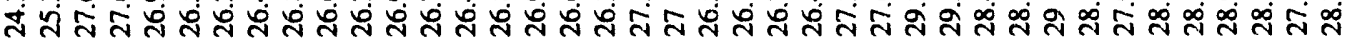

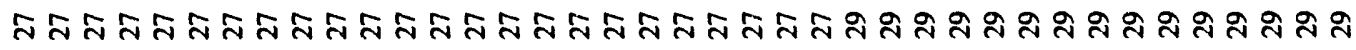

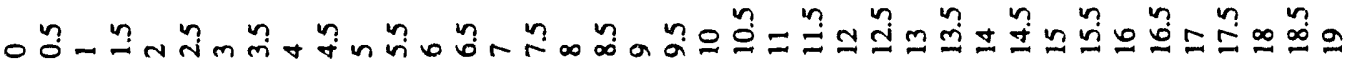


产

曾

总

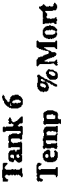

尊

完

喜

a

亭

善高

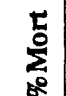

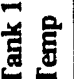

西

๓ั

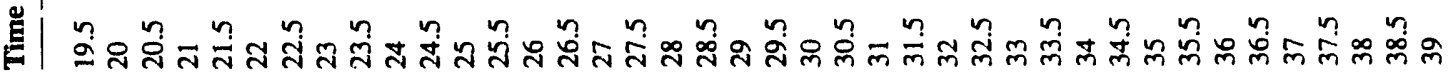

으으으으응ㅇ으으으으으으으으으으으으으으으으으으으으으으으으으으으으으으으으으

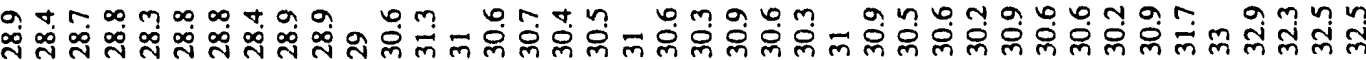

0000000000000000000000000000000000000000

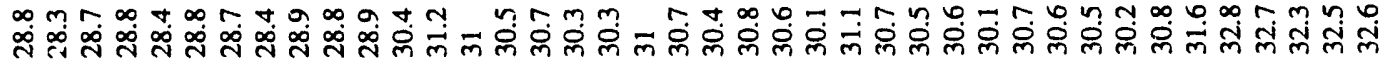

00000000000 으으으으으으으으으으으으으으으으으으으으으으으으

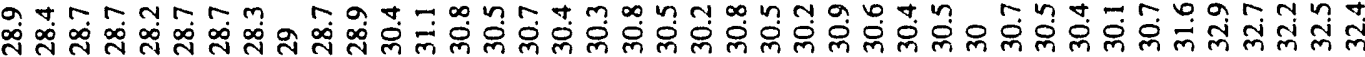

0000000000000000000000000000000000000000

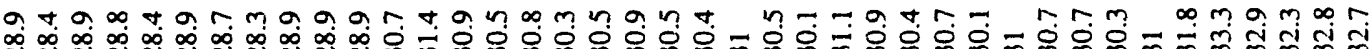

0000000000000000000000000000000000000000

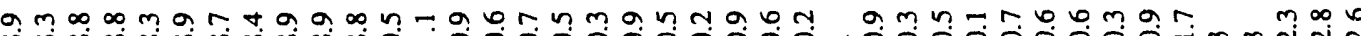

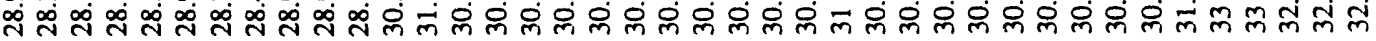

0000000000000000000000000000000000000000

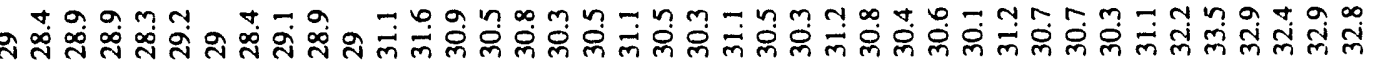
0000000000000000000000000000000000000000

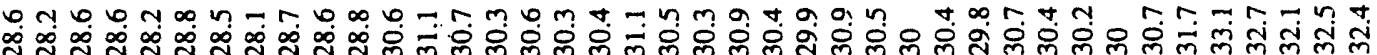
000000000 으으으으으으으으으으으으으으으으으으으으으으으으으으

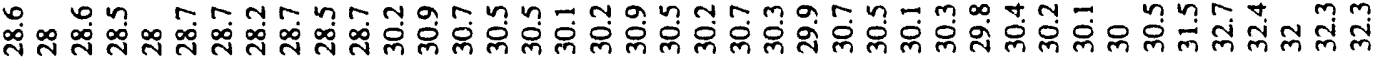

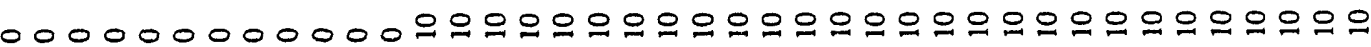
జై సิ సి సి సి సి 
으으으으으으으으으으으으으으으으으으으으으으으으으으으으으으으으으으으으으응 ఇ゙ 0000000000000000000000000000000000000000 总

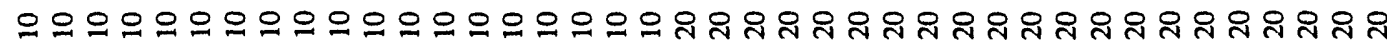

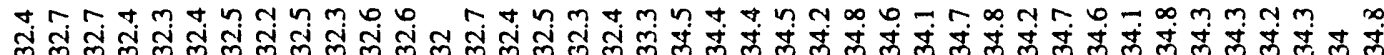

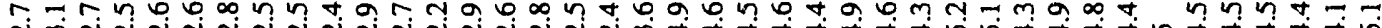

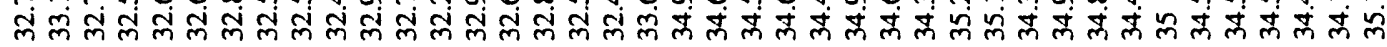

00000000000000000 으으으으으으으으으응ㅇ으으으으으

我

0000000000000000000000000000000000000000

क

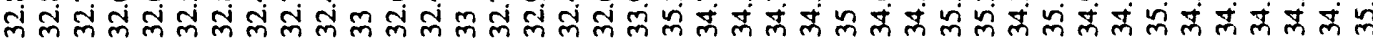

0000000000000000000000000000000000000000

ทำ

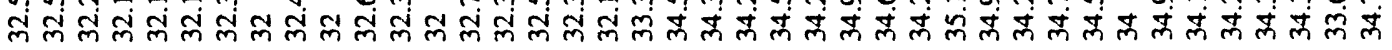

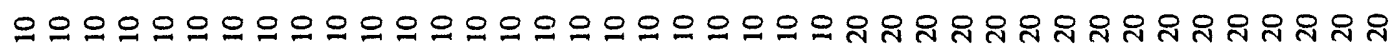
N

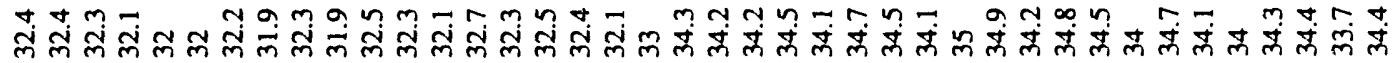

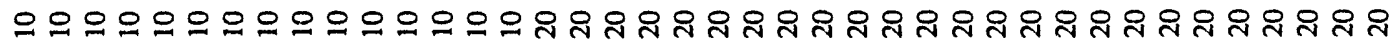

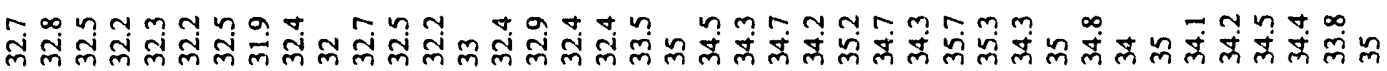


商

首高

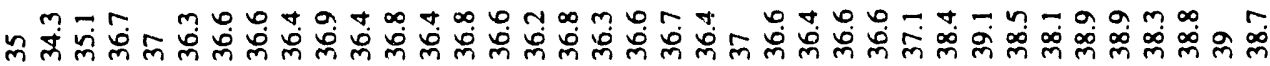

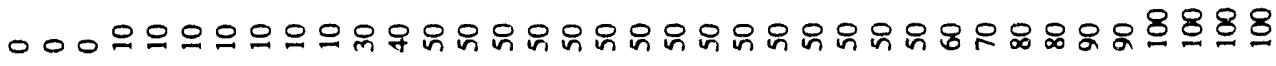

$\infty$

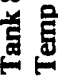

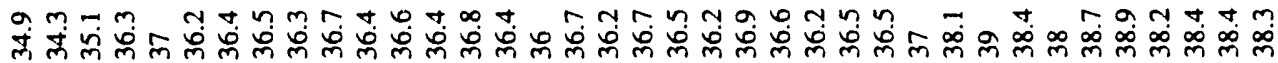

突

尊育

ล0.

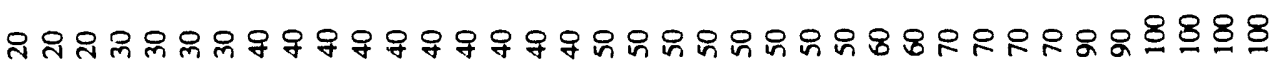

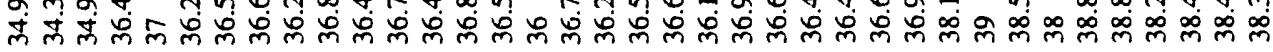

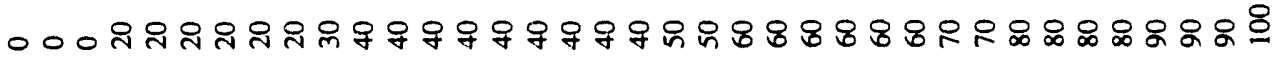

$\infty 8$

善言

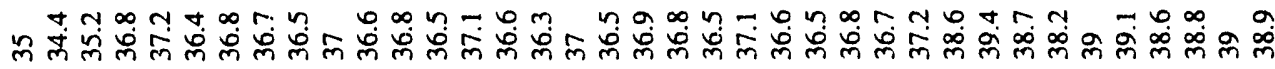

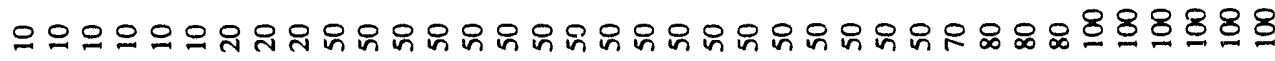

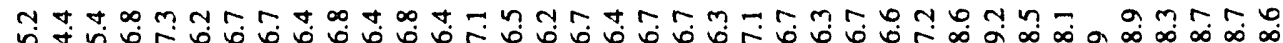

总总

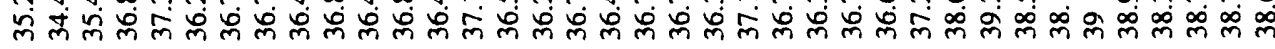

$\sum_{\substack{0 \\ 0}}^{5}$

煎兽

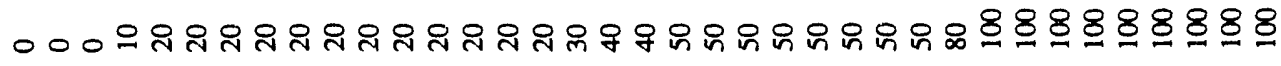

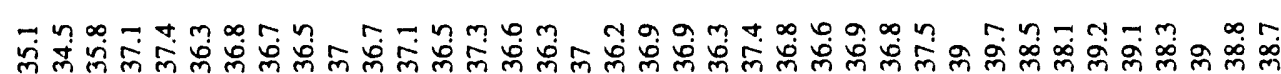

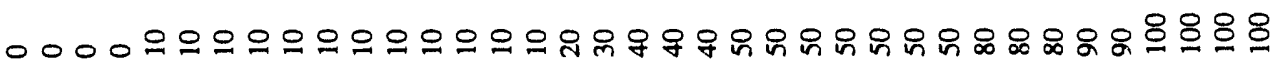

ริ

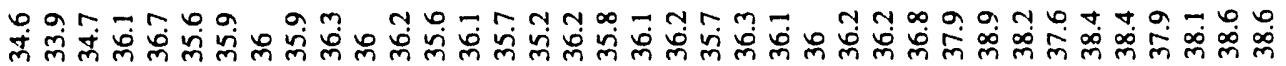

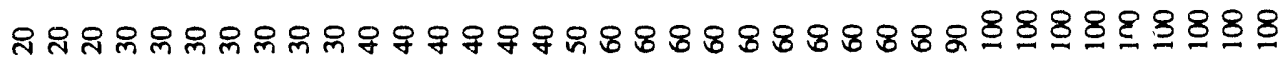

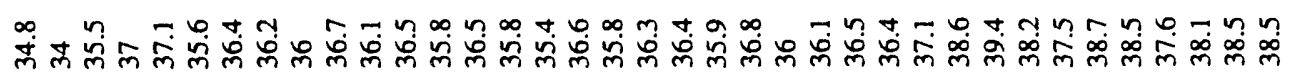

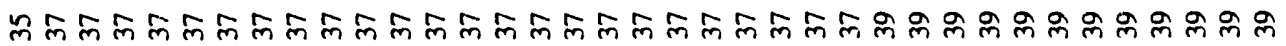

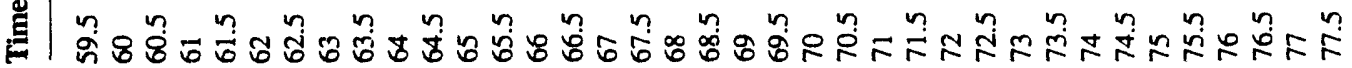


000000000000000000000000000

๙

000000000000000000000000000

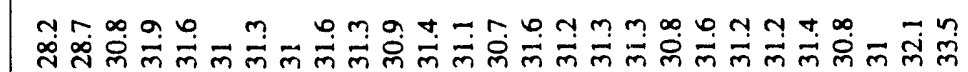

000000000000000000000000000

r

ஸุ่

000000000000000000000000000

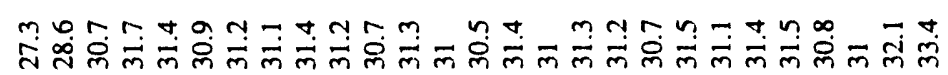

000000000000000000000000000

in

尊要

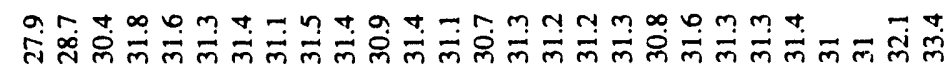

- 00000000000000000000000000 .

熈总

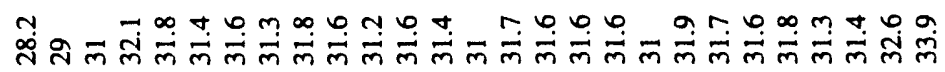

on

000000000000000000000000000

ते

0000 으으오으으으으으으으으으으으으으으으으으으으으

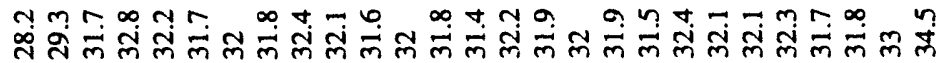

000000000000000000000000000

臨魯

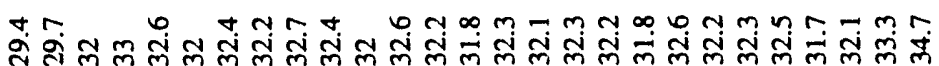

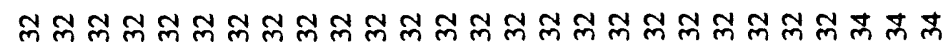

ํㅕㄹ 


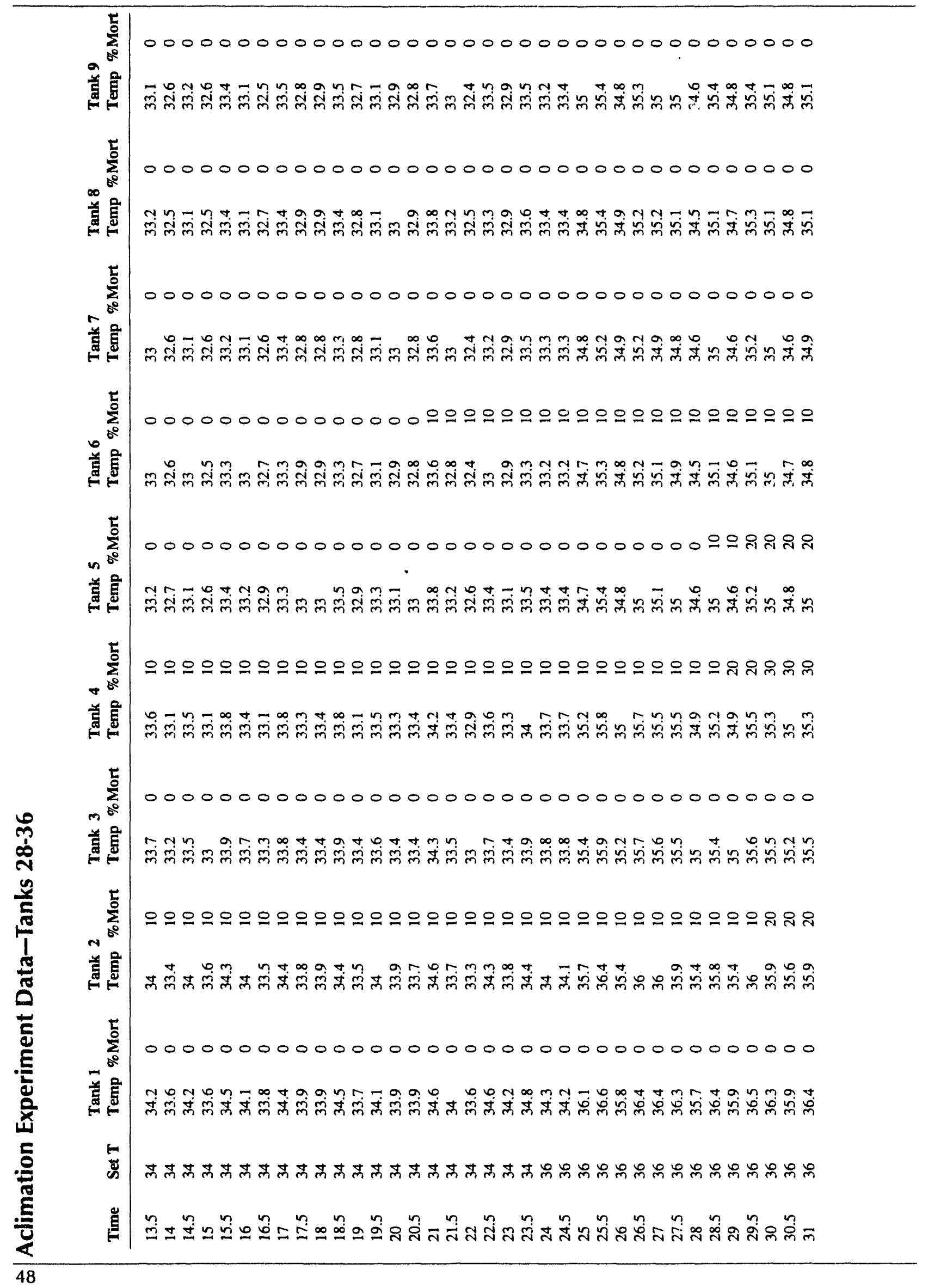




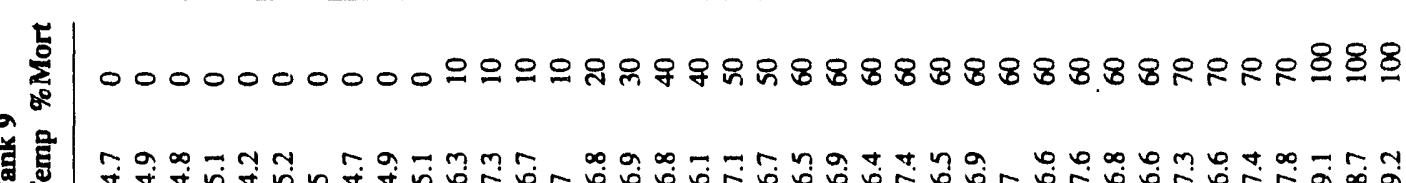

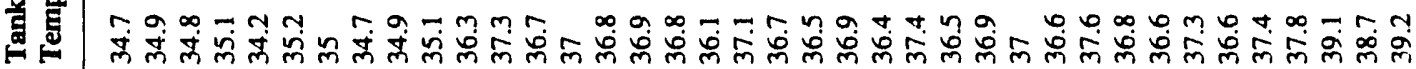

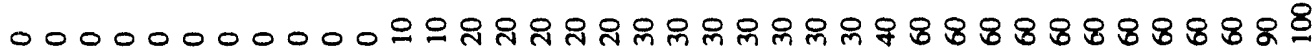

煎員

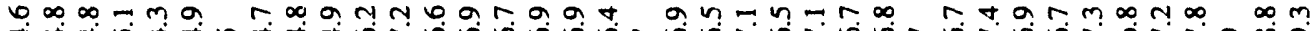

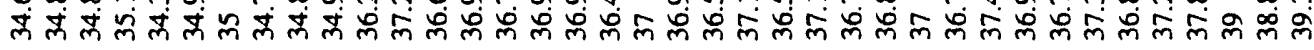

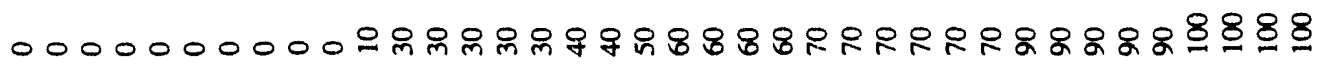

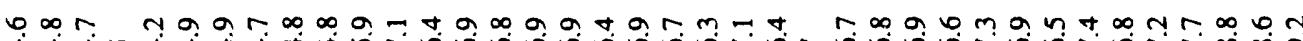

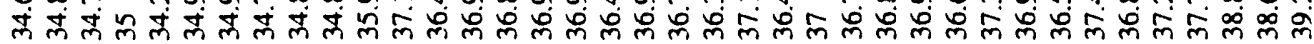

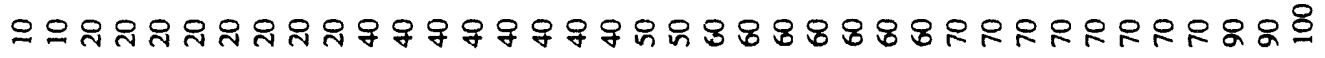

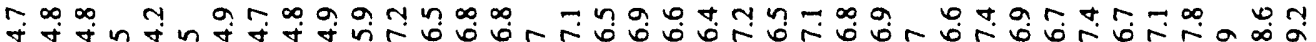

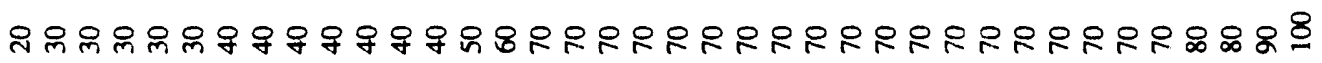

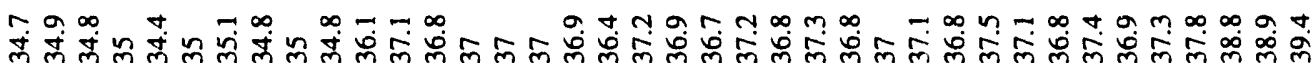

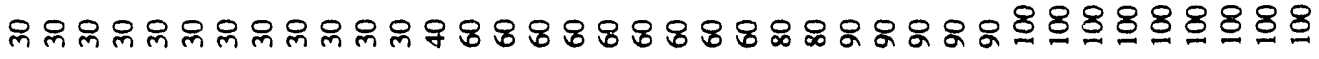

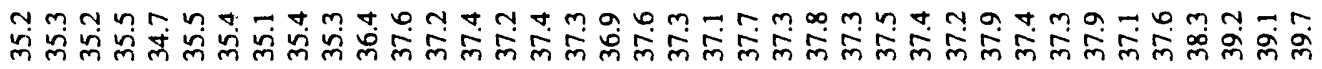

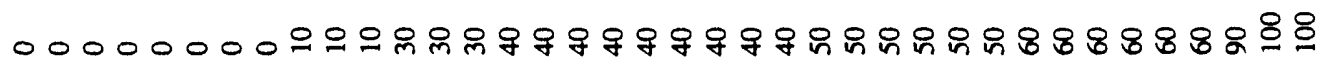

崫会

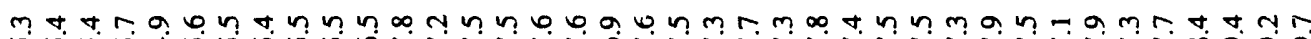

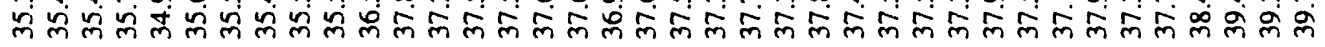

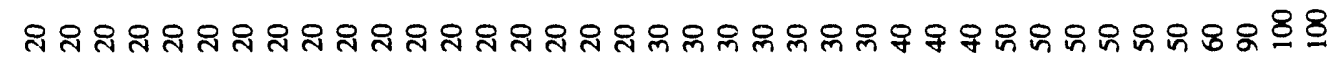

$\omega^{\circ}$

总朂

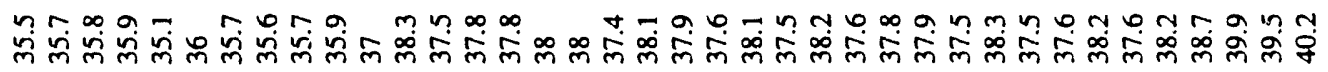

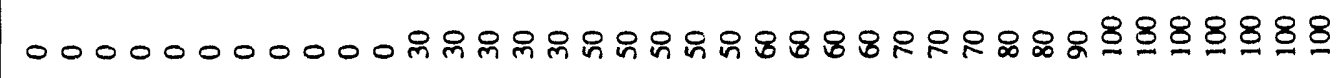

㮍喜

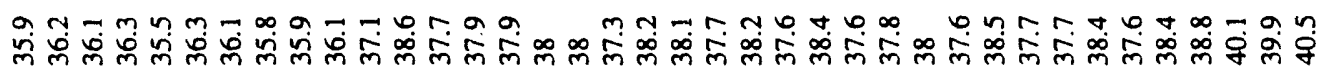

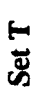

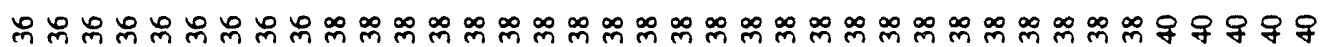

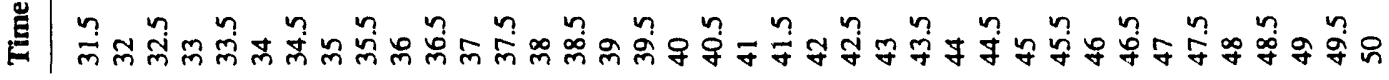




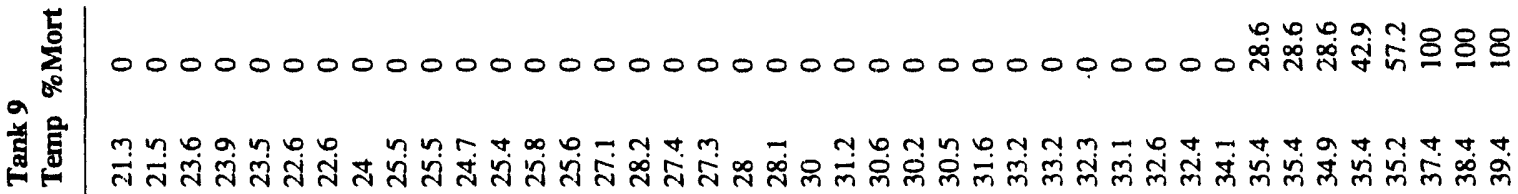

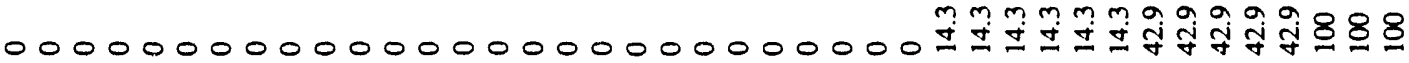

密

mot

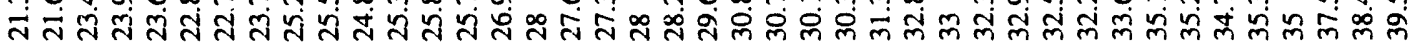

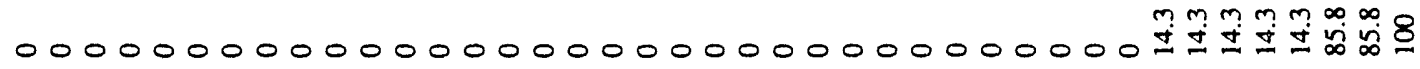

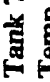

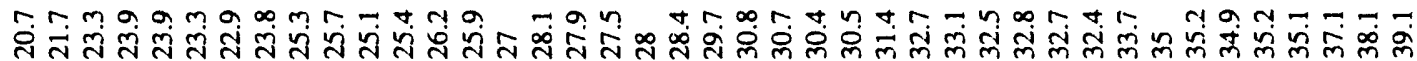

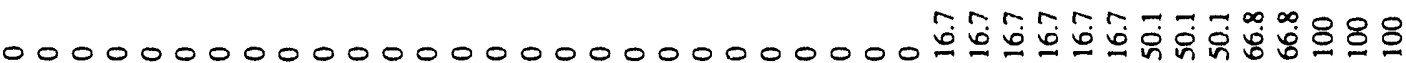

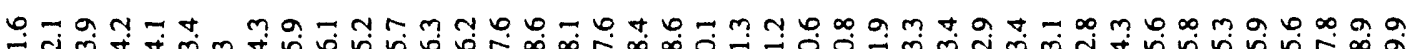

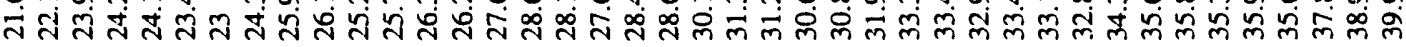

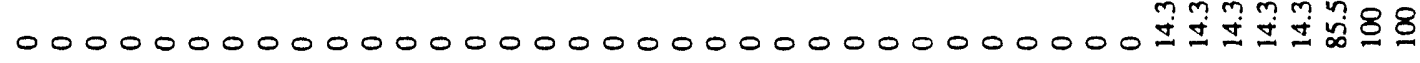

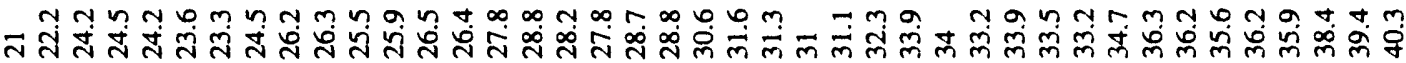

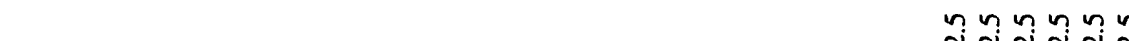
$000000000000000000000000000 \pm N M N$ I సं तี่

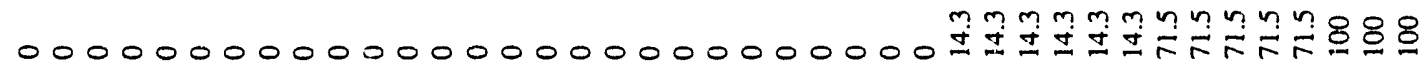

巻 0 冒 0 -

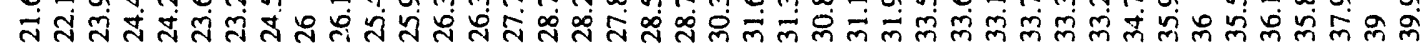

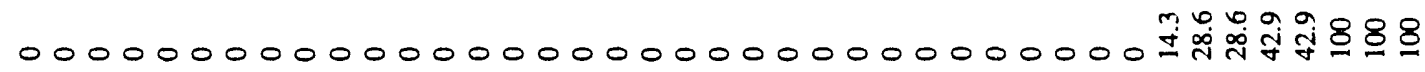
$N$ 菏 000000000000000000000000000 ฟู

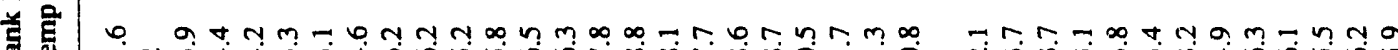

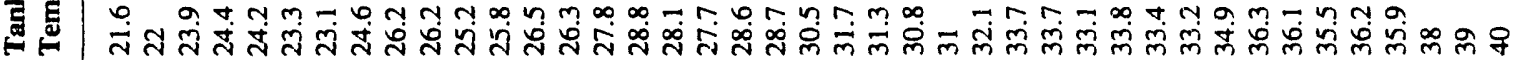

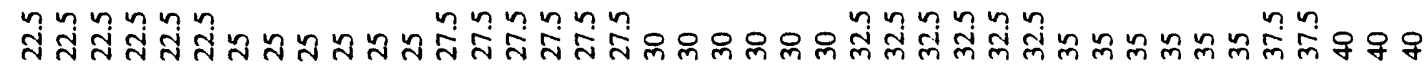
品- 
00000000000000000000000000

息育

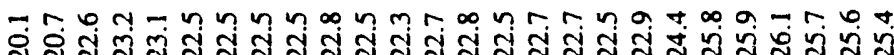

$00000000000000000=0000000$

要望

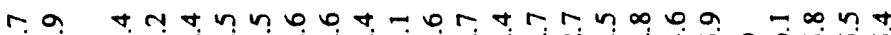

สิ่

00000000000000000000000000

$r$

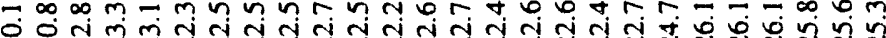

คำ

00000000000000000000000000

$\infty$

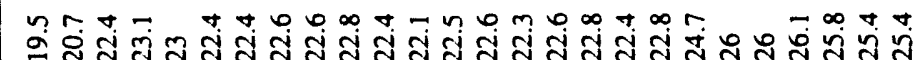

00000000000000000000000000

in

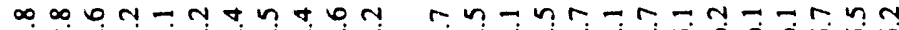

อง

00000000000000000000000000

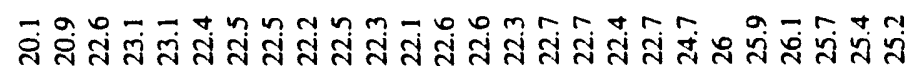

00000000000000000000000000

鸢量

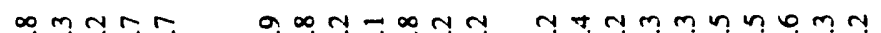

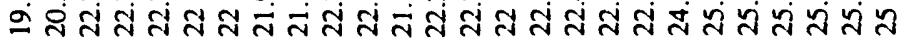

000000000000 으으으으으응ㅇㅇㅇㅡ

n

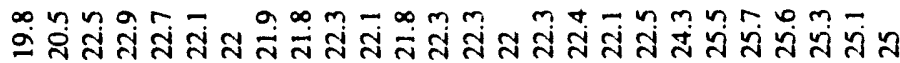

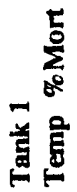

00000000000000000000000000

бํำ

*

๖ ส

Eี 


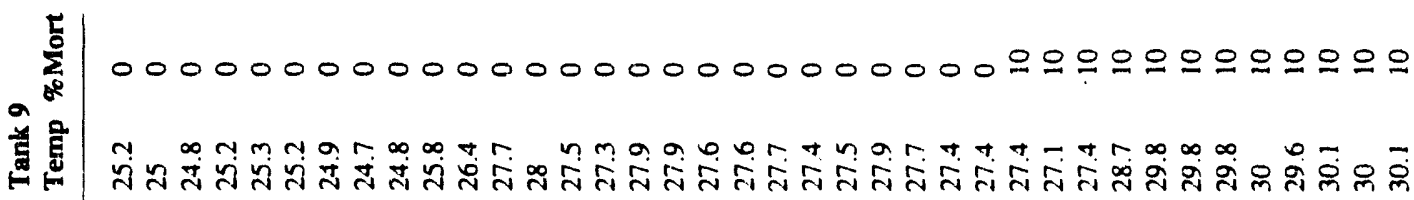
尊

00000000000000000000000000000000000000

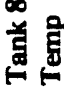

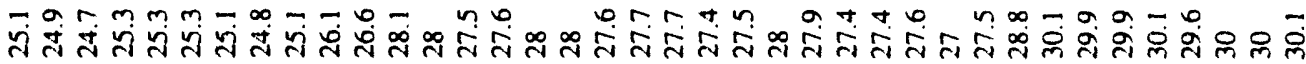

00000000000000000000000000000000000000

㮍曷

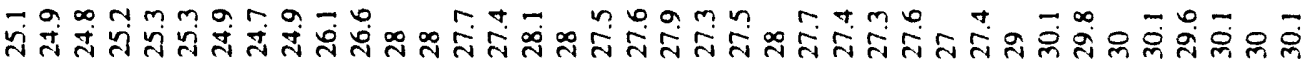

0000000000000 으으으으으으으으으응ㅇㅇㅇㅇㅇㅇㅇ으으으으으으

总兽

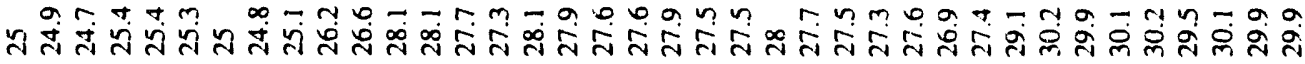

00000000000000000000000000000000000000

T-

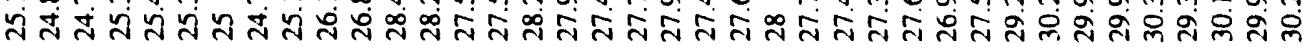

000000000000000004.00000000000000000000

鸢晨

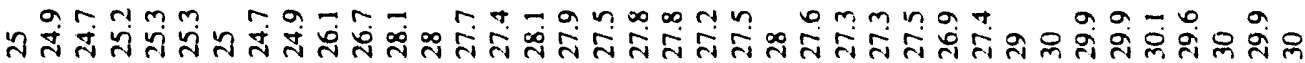

00000000000000000000000000000000000000

恶焉

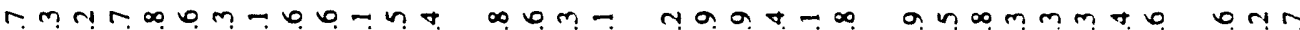

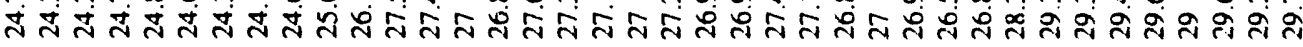

으으으으으으으으으으으으으으으으으으으으으으으으으으으으으으으응ㅇㅇㅡ $n$

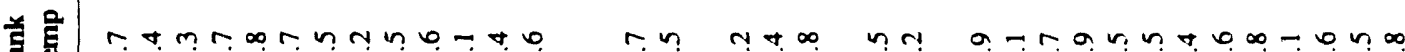

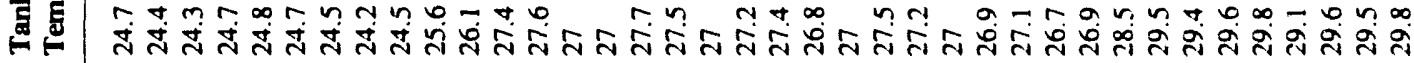

窟

00000000000000000000000000000000000000

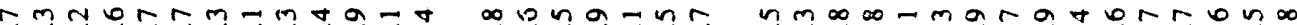

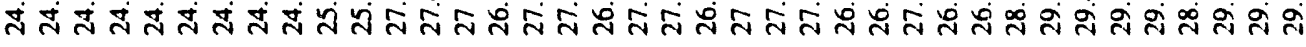

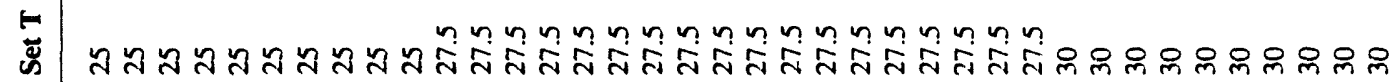

帝| 


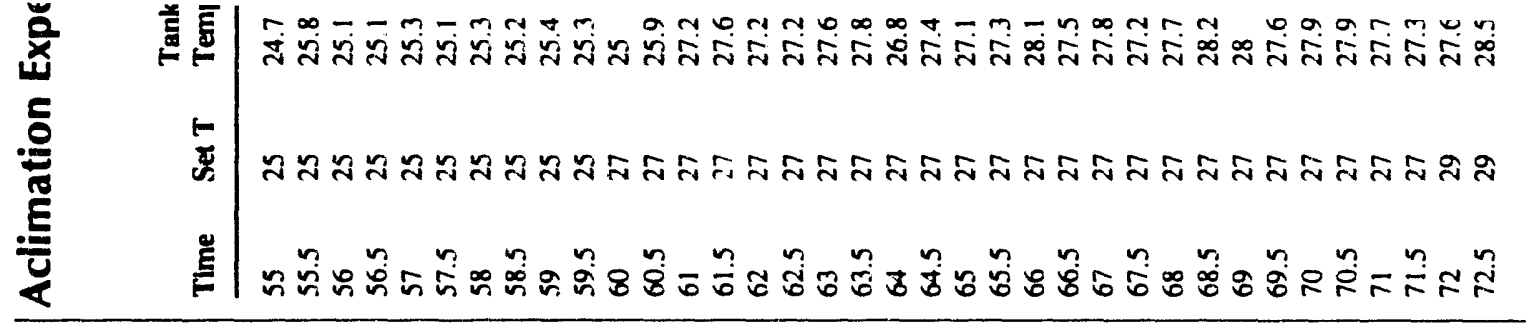

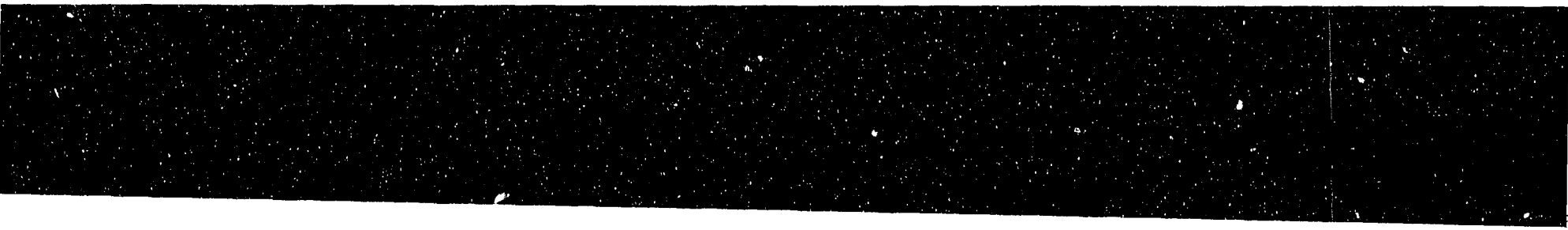

Response of Fish to Different Simulated Rates of Water Temperature Increase

으으으으으으으으으으으으으으으으으으으응ㅇㅇㅇㅇ으으으으으으으으으

ลें

$\infty$

000000000000000000000000000000000 ำ

总兽

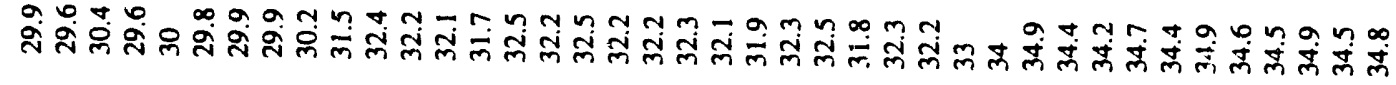

監

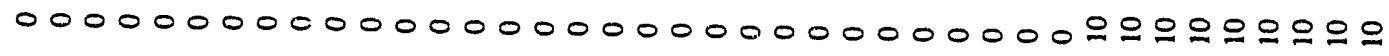

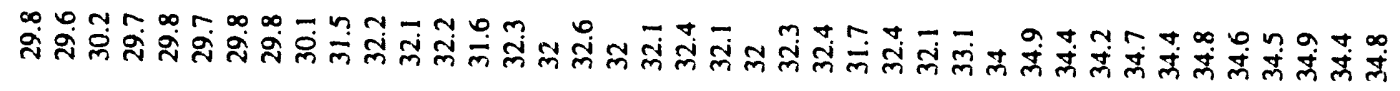

总窟

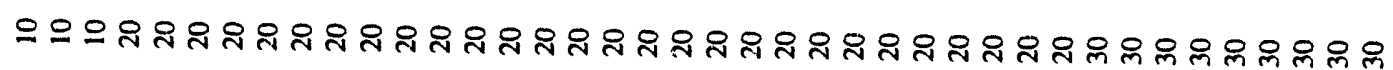

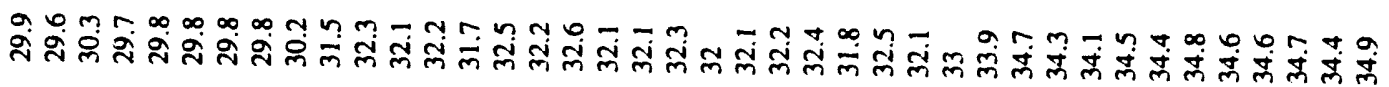

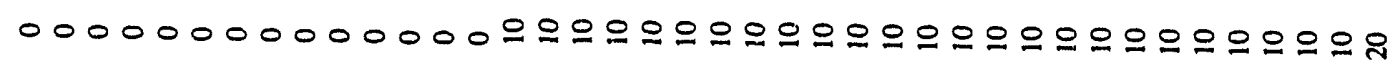

䆠兽

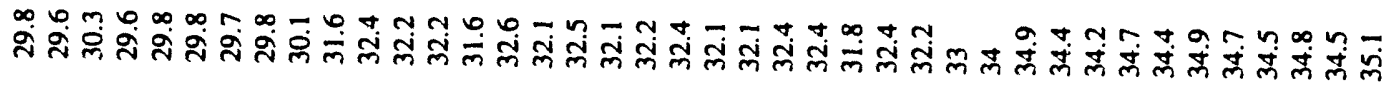

0000000000000000000000000000000000000000

豎昜

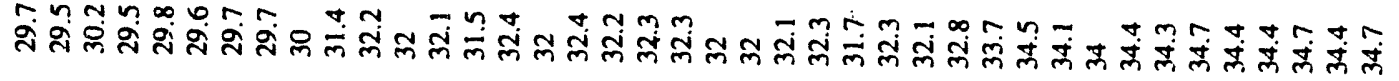

$\sum_{0}^{5}$

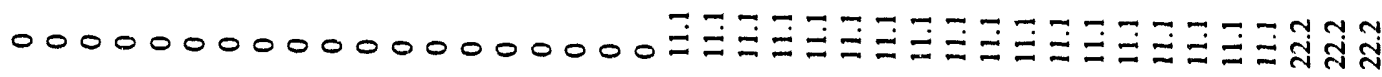

总兽

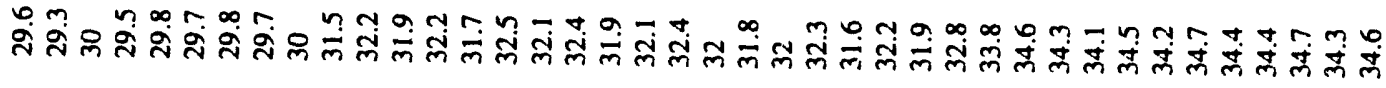

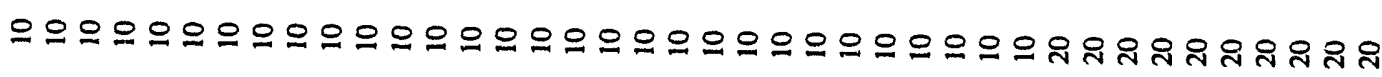

\%

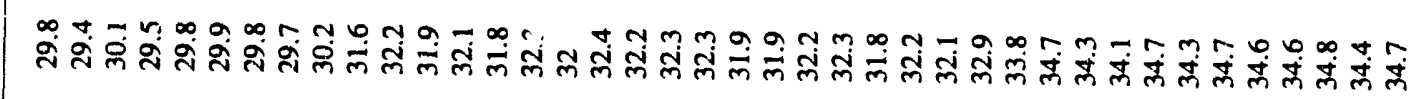

0000000000000000000000000000000000 vivinivivivi

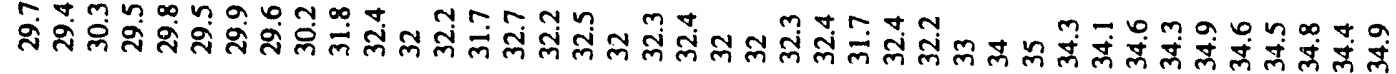

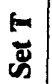

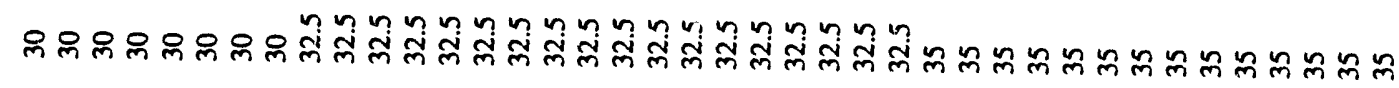

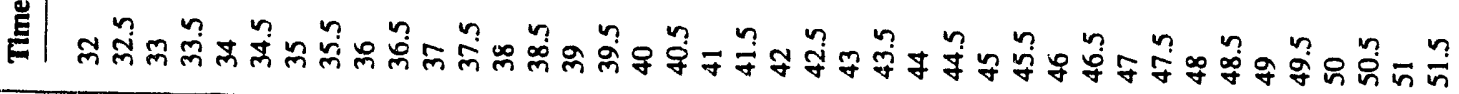


善

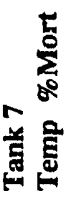

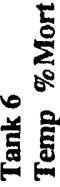

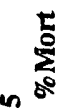

兽育

善育悹

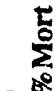

善言

48

善言

놀

总言

范

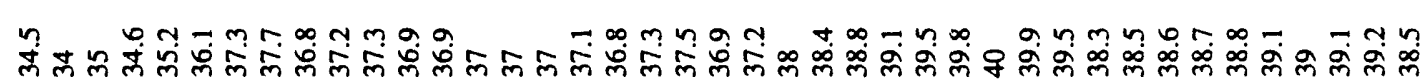

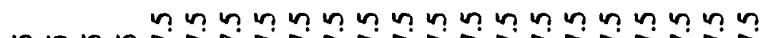

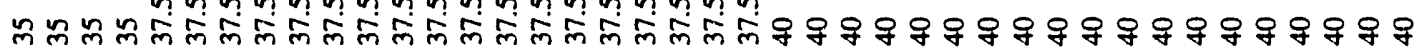

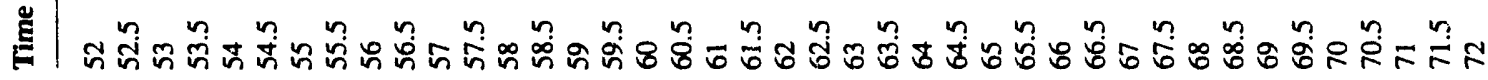

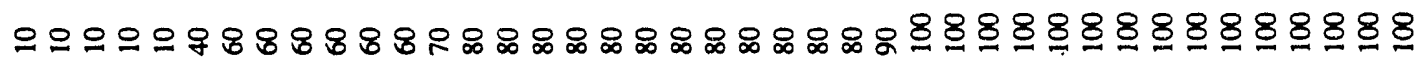

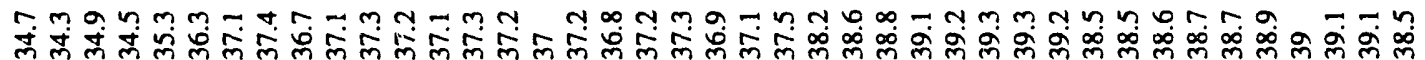

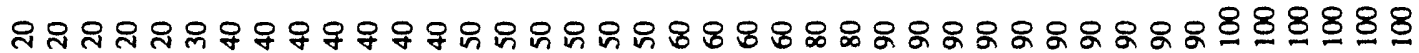

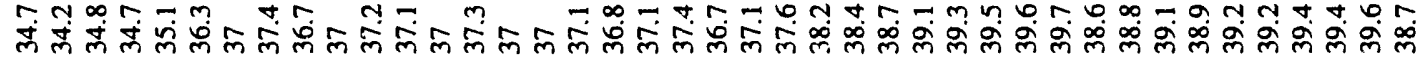

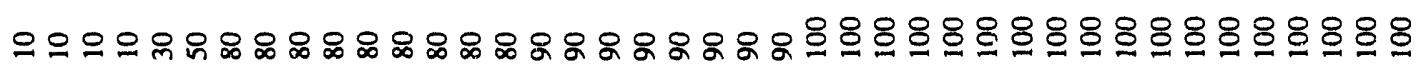

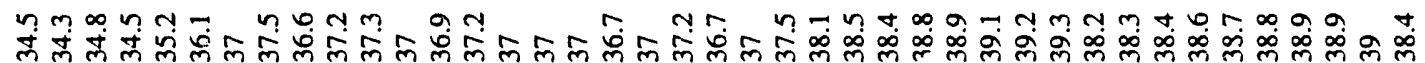

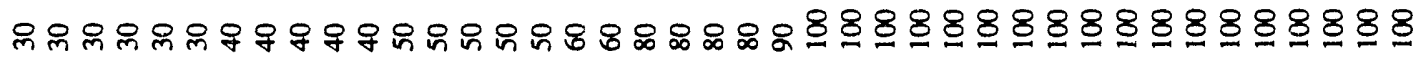

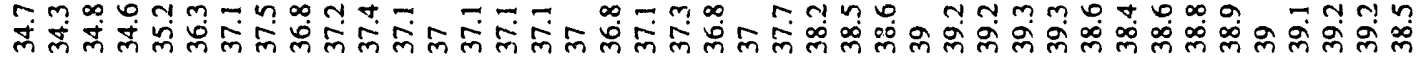

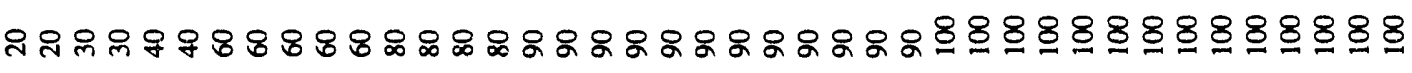

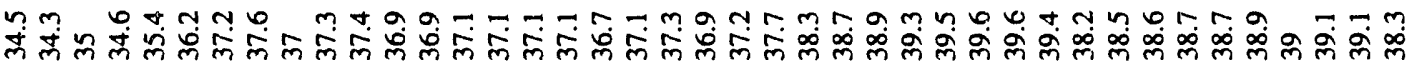

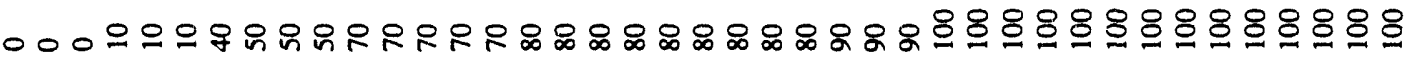

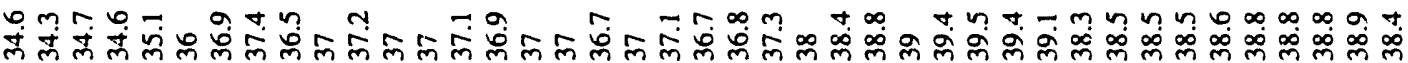

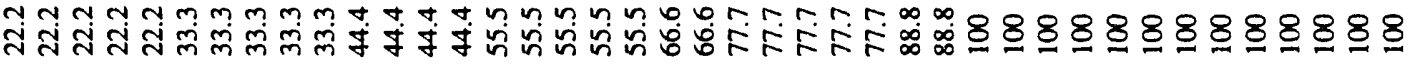

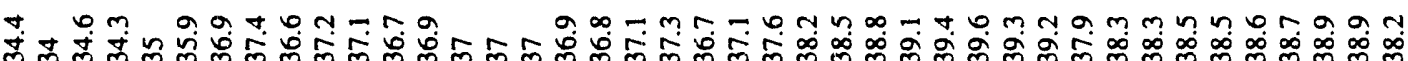

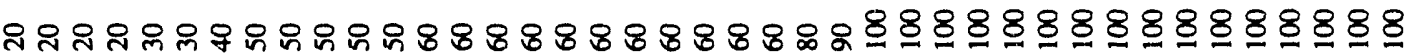

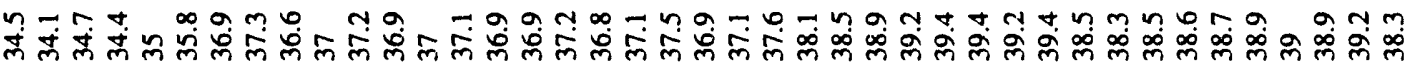

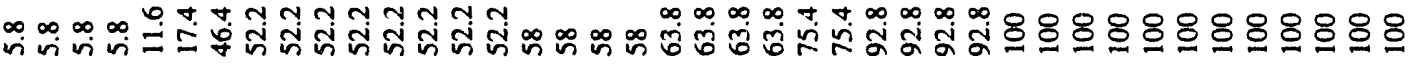


0000000000000000000000000000

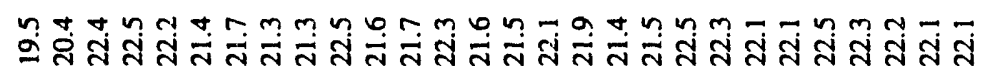

0000000000000000000000000000

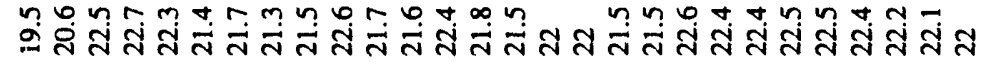

0000000000000000000000000000

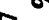

פ̊ำ

0000000000000000000000000000

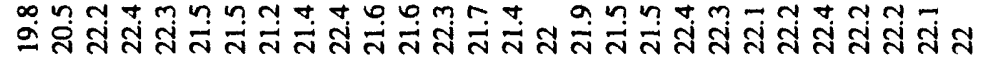

0000000000000000000000000000

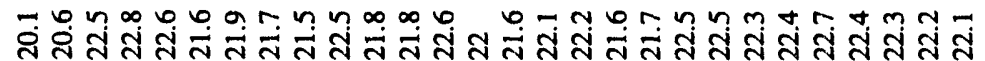

0000000000000000000000000000

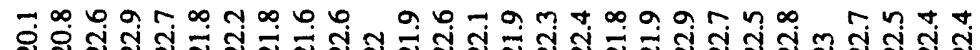

0000000000000000000000000000

$\infty$

惡墨

0000000000000000000000000000

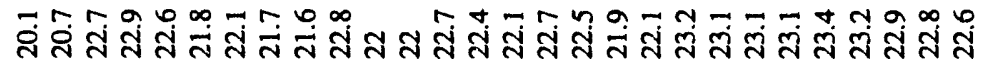

0000000000000000000000000000

B)

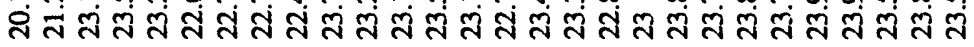

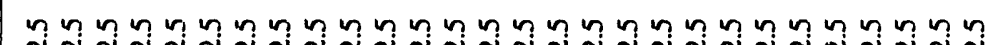

สำ ส

ํㅕㅂ 
00000000000000000000000000000000000000

를

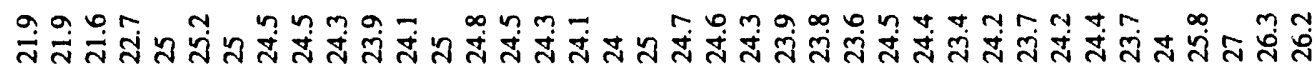

00000000000000000000000000000000000000

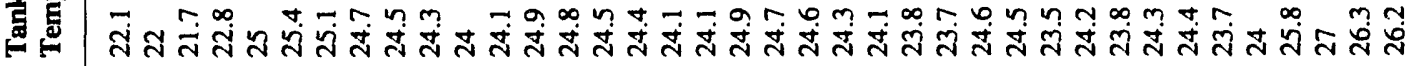

00000000000000000000000000000000000000

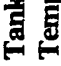

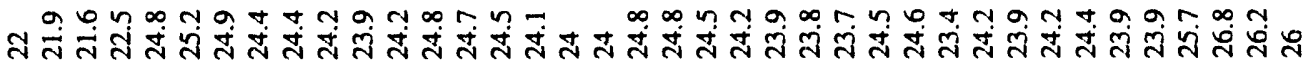

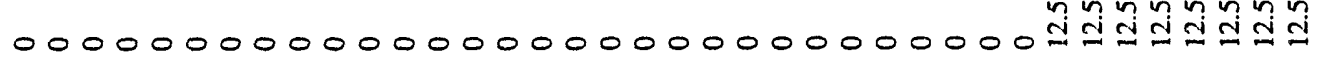

ส ส สง

00000000000000000000000000000000000000

nd

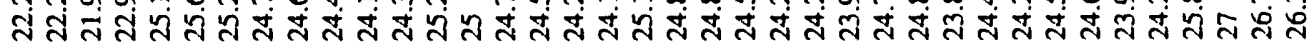

00000000000000000000000000000000000000

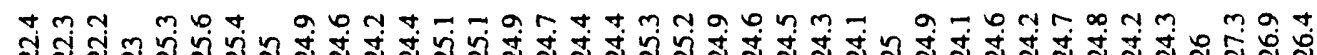

สูง

00000000000000000000000000000000000000

สี่

00000000000000000000000000000000000000 $N$

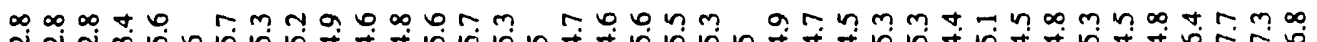

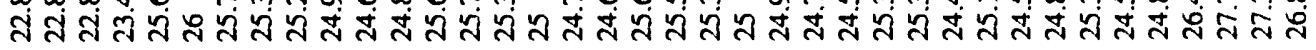
00000000000000000000000000000000000000 羔号

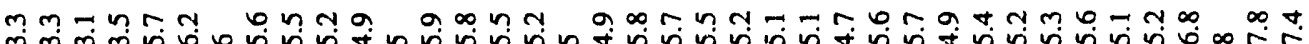

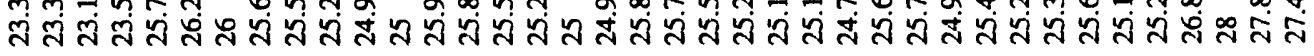




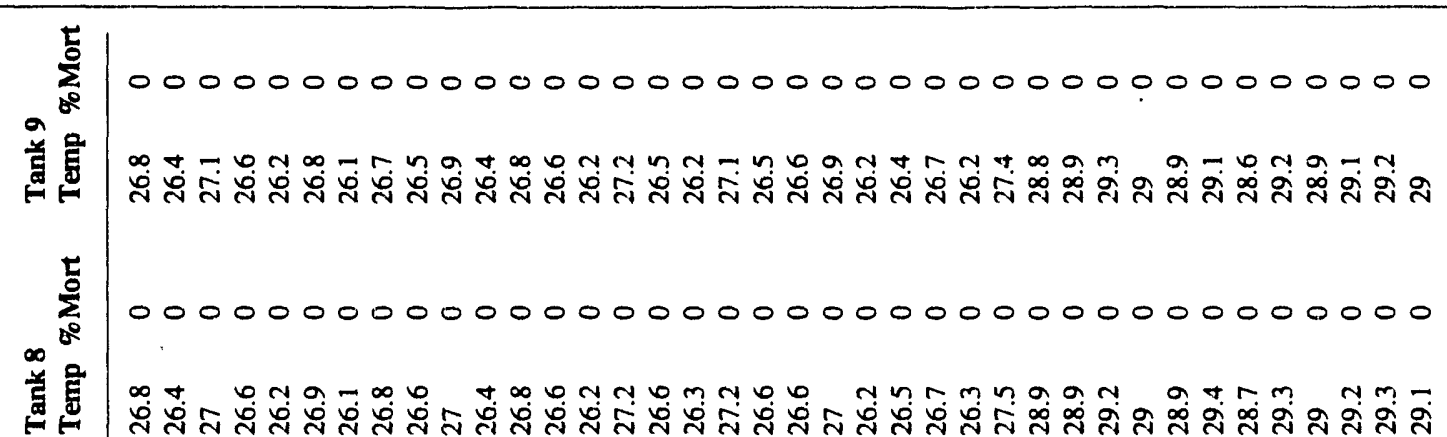

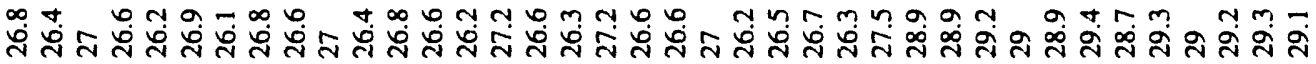
00000000000000000000000000000000000000 m m

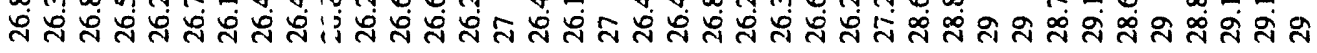

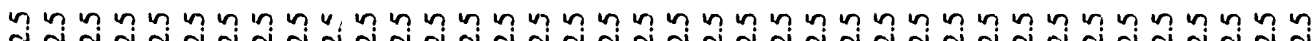

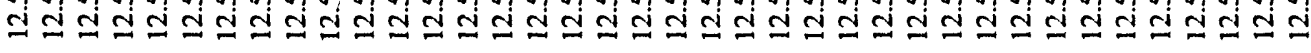
6

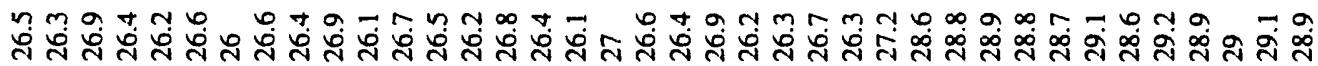
00000000000000000000000000000000000000 in 8

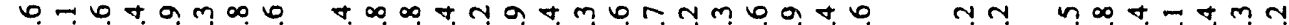

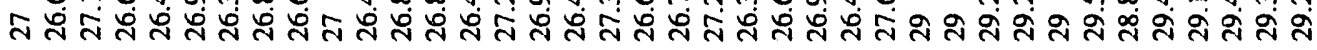
00000000000000000000000000000000000000 $-8$

ๆ $00000000000000000000000000000000 a 9 a 9 a 0$ nar.

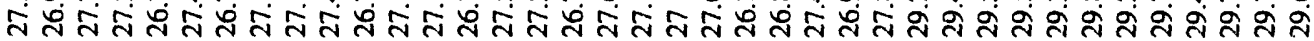

00000000000000000000000000000000000000

0. m nat n

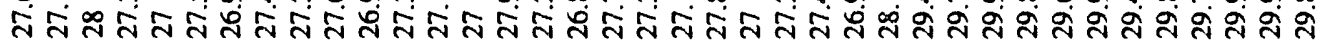
00000000000000000000000000000000000000

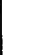
तூ

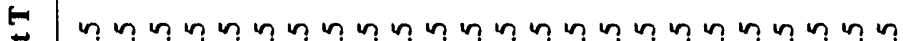

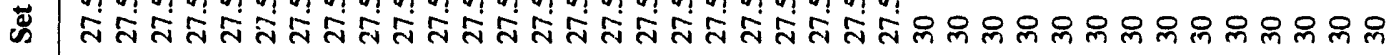

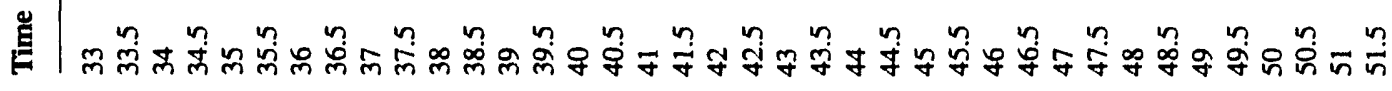




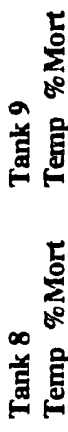

駡

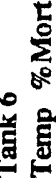

党

善言

$\sum_{0}^{5}$

을 을

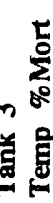

0000000000000000000000000000000000000 茫

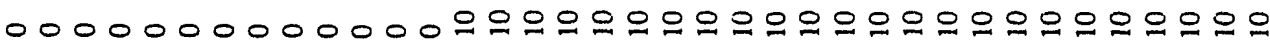

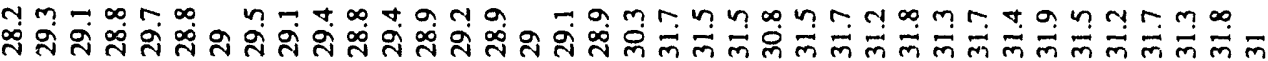
0000000000000000000000000000000000000 ஸ்

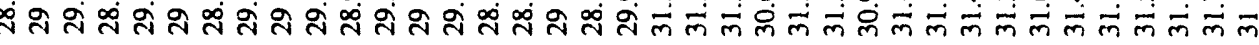

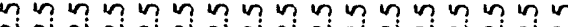

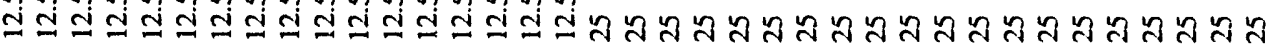
ก $\quad$ n

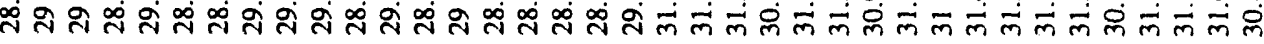
0000000000000000000000000000000000000 षัษต

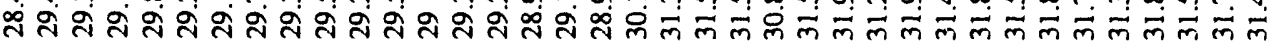
0000000000000000000000000000000000000 क्ष a a a a a a a a a a a a a a a a a a a a a a a a a a a a a a कं 0000000000000000000000000000000000000

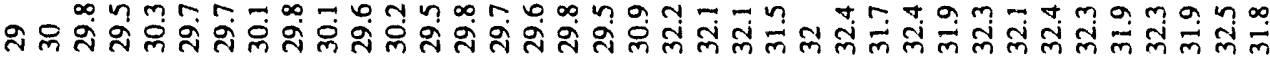
0000000000000000000000000000000000000

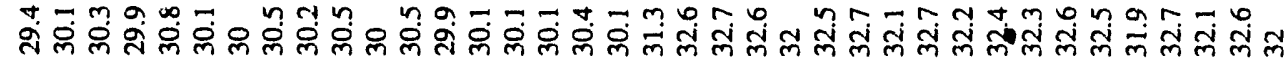

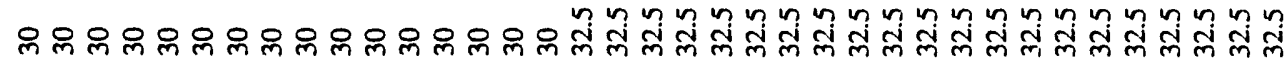

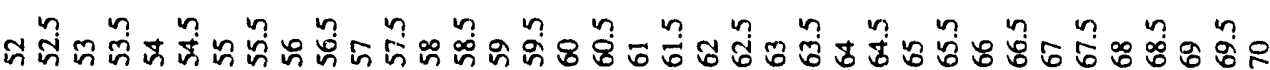




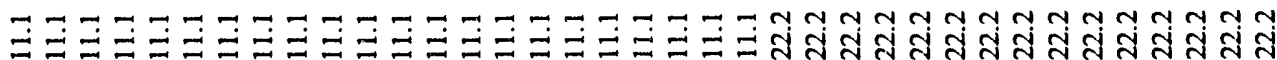

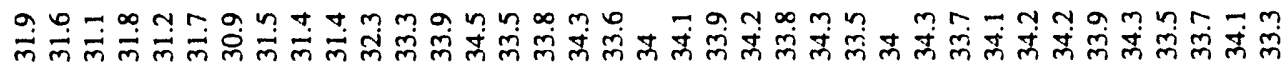

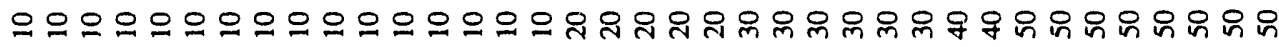

駦

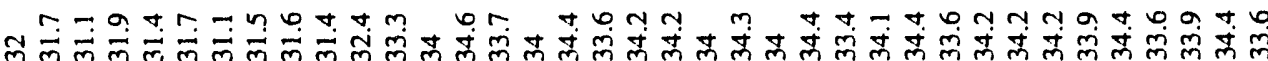

000000000000 으으으으으으으으으으으으으으으으으으으으으으으으으

惫空

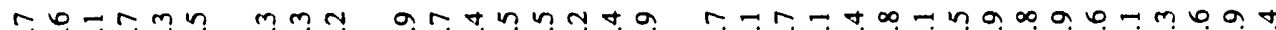

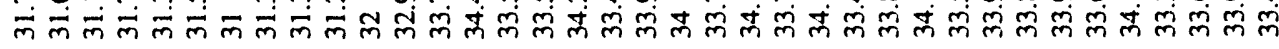

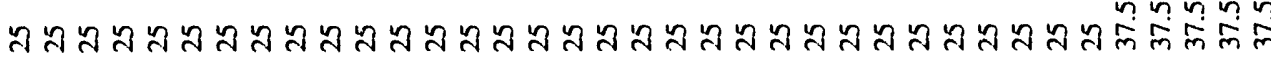

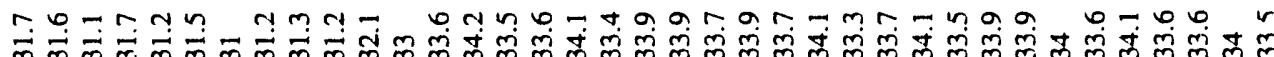

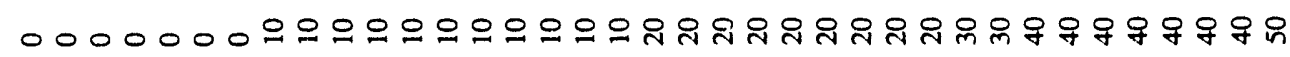

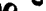

芫鄫

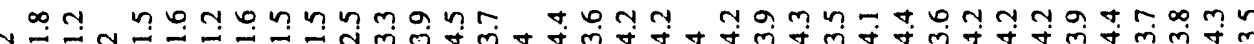

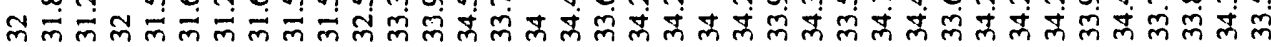
$000000000000000000000000000000000=\Xi=$

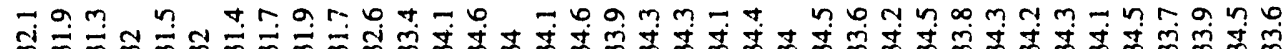

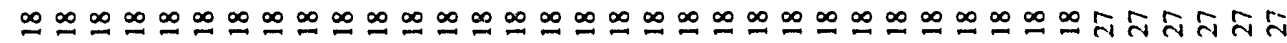

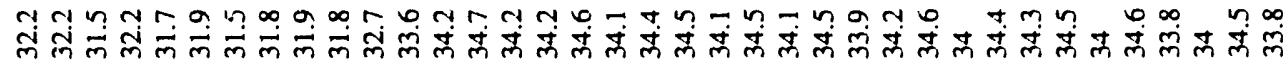

0000000000000000000 으으으으으으으으으으으으으으

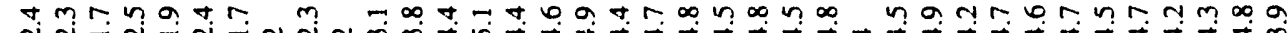

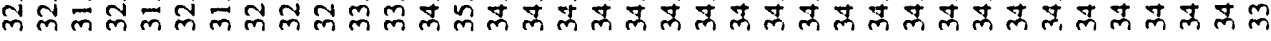

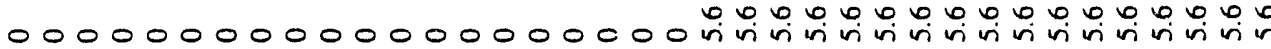

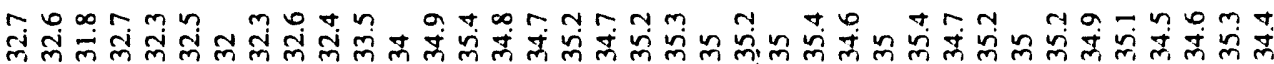

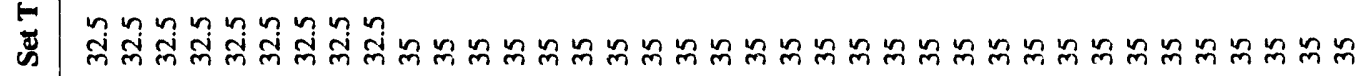

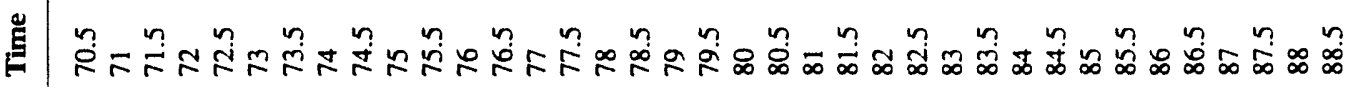



索

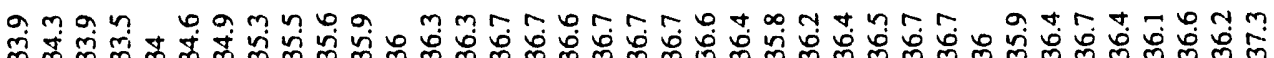

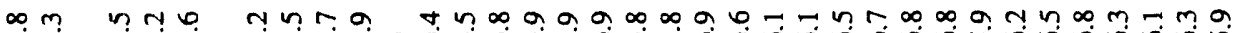

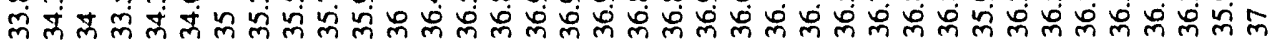

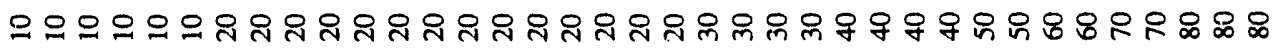

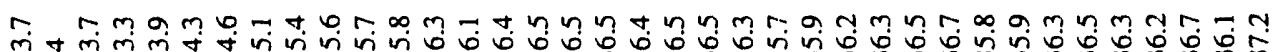

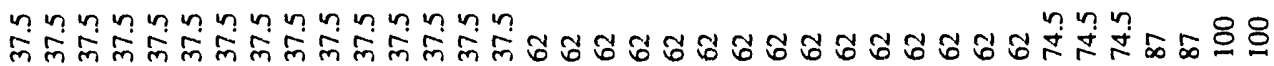

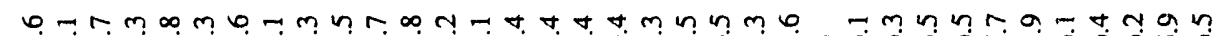

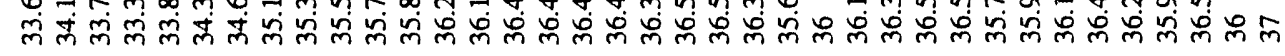

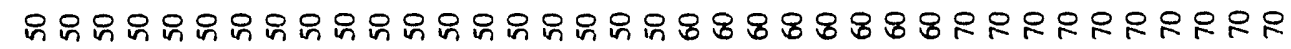

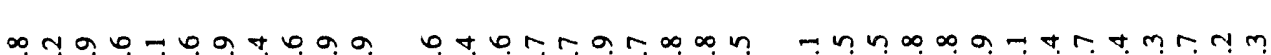

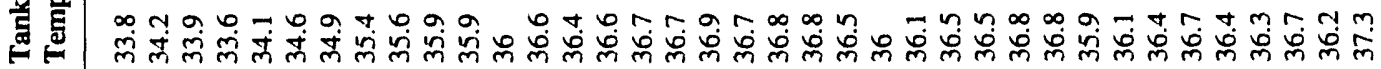

$\sum_{0}^{5}$

䓛亮

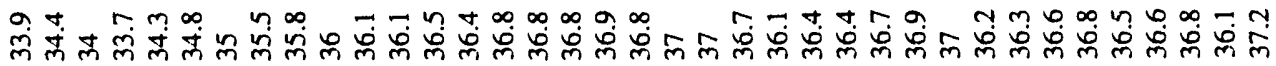

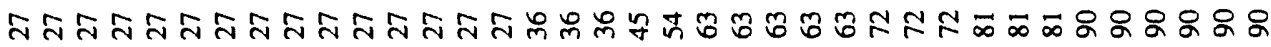

䓛

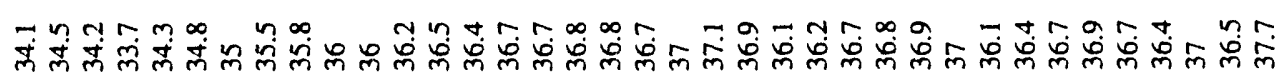

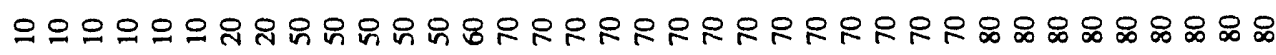

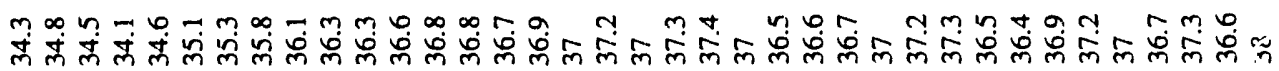

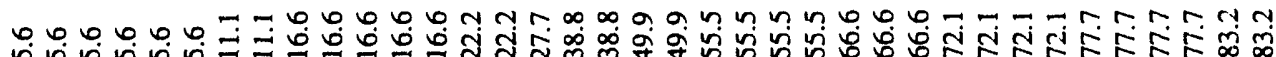

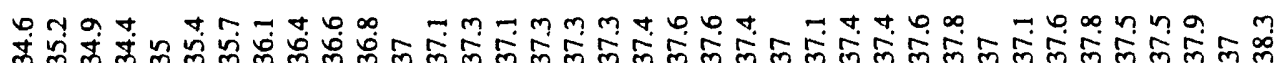

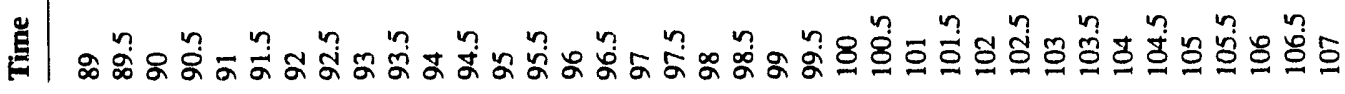




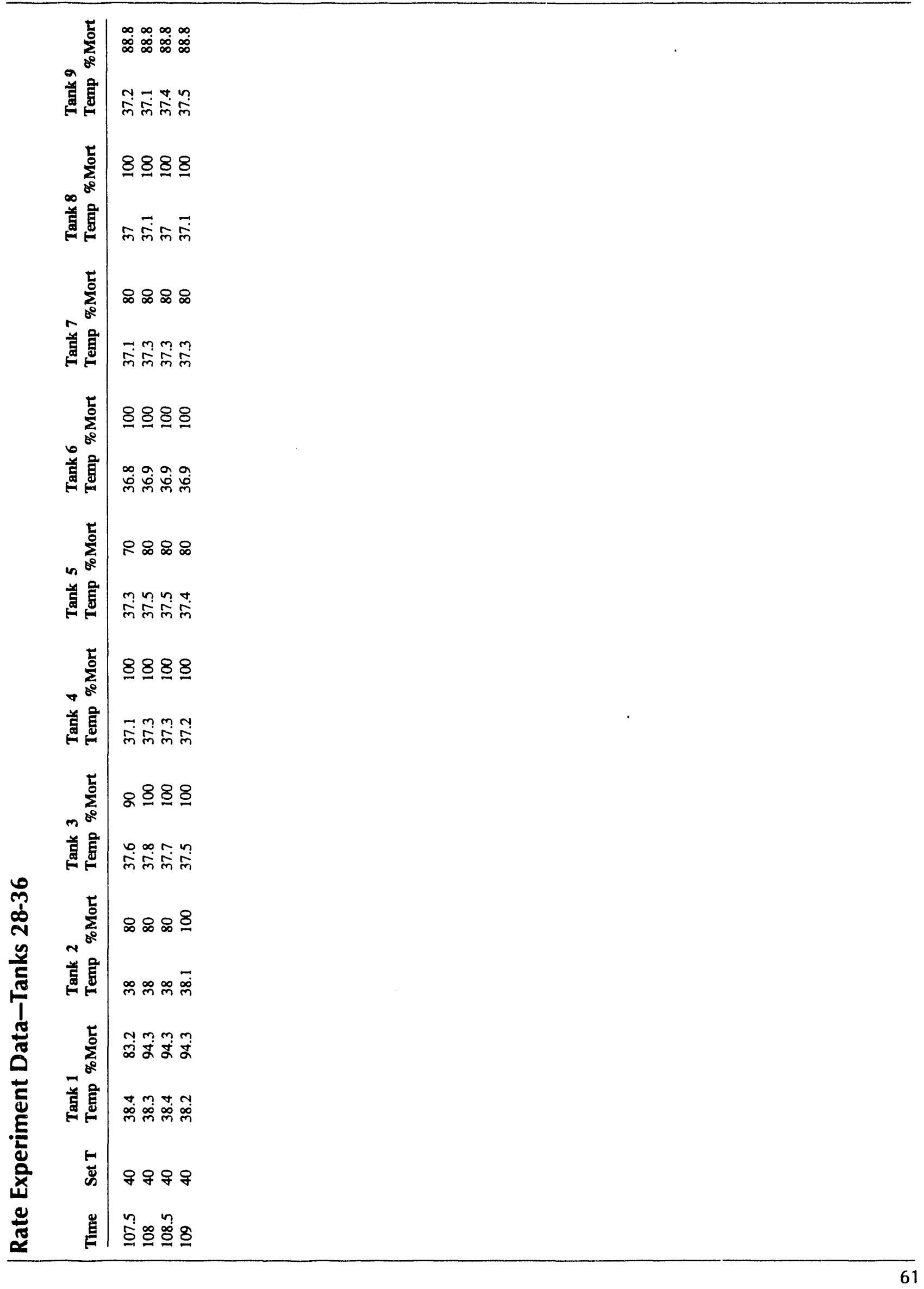


Appendix C 


\section{Rate Experiment Logits}

\begin{tabular}{rrlrrrll} 
RowNum & Expt & \multicolumn{2}{l}{ TTCum Deaths } & Deaths & Num @ Risk & q & p \\
\hline 1 & 1 & 22.5 & 0 & 0 & 72 & 0.005 & 0.995 \\
2 & 1 & 25 & 0 & 0 & 72 & 0.005 & 0.995 \\
3 & 1 & 27.5 & 0 & 0 & 72 & 0.005 & 0.995 \\
4 & 1 & 30 & 0 & 0 & 72 & 0.005 & 0.995 \\
5 & 1 & 32.5 & 0 & 0 & 72 & 0.005 & 0.995 \\
6 & 1 & 35 & 6 & 6 & 72 & 0.08333333 & 0.91666667 \\
7 & 1 & 37.5 & 35 & 29 & 66 & 0.43939394 & 0.56060606 \\
8 & 1 & 40 & 72 & 37 & 37 & 0.995 & 0.005 \\
9 & 2 & 22.5 & 0 & 0 & 95 & 0.005 & 0.995 \\
10 & 2 & 25 & 1 & 1 & 95 & 0.01052632 & 0.98947368 \\
11 & 2 & 27.5 & 1 & 0 & 94 & 0.005 & 0.995 \\
12 & 2 & 30 & 3 & 2 & 94 & 0.0212766 & 0.9787234 \\
13 & 2 & 32.5 & 4 & 1 & 92 & 0.01086957 & 0.98913043 \\
14 & 2 & 35 & 5 & 1 & 91 & 0.01098901 & 0.98901099 \\
15 & 2 & 37.5 & 19 & 14 & 90 & 0.15555556 & 0.84444444 \\
16 & 2 & 40 & 95 & 76 & 76 & 0.995 & 0.005 \\
17 & 2 & 22.5 & 0 & 0 & 87 & 0.005 & 0.995 \\
18 & 3 & 25 & 0 & 0 & 87 & 0.005 & 0.995 \\
19 & 3 & 27.5 & 1 & 1 & 87 & 0.01149425 & 0.98850575 \\
20 & 3 & 30 & 1 & 0 & 86 & 0.005 & 0.995 \\
21 & 3 & 32.5 & 2 & 1 & 86 & 0.01162791 & 0.98837209 \\
22 & 3 & 35 & 5 & 3 & 85 & 0.03529412 & 0.96470588 \\
23 & 3 & 37.5 & 19 & 14 & 82 & 0.17073171 & 0.82926829 \\
24 & 3 & 40 & 87 & 68 & 68 & 0.995 & 0.005
\end{tabular}




\section{Acclimation Experiment Logits}

\begin{tabular}{|c|c|c|c|c|c|c|c|}
\hline RowNum & Expt & TTCun & Deaths & Deaths & Num @ Risk & $\mathbf{q}$ & $\mathbf{p}$ \\
\hline 1 & 1 & 22 & 0 & 0 & 94 & 0.005 & 0.995 \\
\hline 2 & 1 & 24 & 0 & 0 & 94 & 0.005 & 0.995 \\
\hline 3 & 1 & 26 & 1 & 1 & 94 & 0.0106383 & 0.9893617 \\
\hline 4 & 1 & 28 & 2 & 1 & 93 & 0.01075269 & 0.98924731 \\
\hline 5 & 1 & 30 & 2 & 0 & 92 & 0.005 & 0.995 \\
\hline 6 & 1 & 32 & 3 & 1 & 92 & 0.01086957 & 0.98913043 \\
\hline 7 & 1 & 34 & 6 & 3 & 91 & 0.03296703 & 0.96703297 \\
\hline 8 & 1 & 36 & 10 & 4 & 88 & 0.04545455 & 0.95454545 \\
\hline 9 & 1 & 38 & 94 & 84 & 84 & 0.36904762 & 0.63095238 \\
\hline 10 & 2 & 17 & 0 & 0 & 90 & 0.005 & 0.995 \\
\hline 11 & 2 & 19 & 0 & 0 & 90 & 0.005 & 0.995 \\
\hline 12 & 2 & 21 & 0 & 0 & 90 & 0.005 & 0.995 \\
\hline 13 & 2 & 23 & 0 & 0 & 90 & 0.005 & 0.995 \\
\hline 14 & 2 & 25 & 0 & 0 & 90 & 0.005 & 0.995 \\
\hline 15 & 2 & 28 & 0 & 0 & 90 & 0.005 & 0.995 \\
\hline 16 & 2 & 30 & 0 & 0 & 90 & 0.005 & 0.995 \\
\hline 17 & 2 & 32 & 2 & 2 & 90 & 0.02222222 & 0.97777778 \\
\hline 18 & 2 & 34 & 6 & 4 & 88 & 0.04545455 & 0.95454545 \\
\hline 19 & 2 & 36 & 9 & 3 & 84 & 0.03571429 & 0.96428571 \\
\hline 20 & 2 & 38 & 28 & 19 & 81 & 0.2345679 & 0.7654321 \\
\hline 21 & 2 & 39 & 90 & 62 & 62 & 0.995 & 0.005 \\
\hline 22 & 3 & 27 & 0 & 0 & 90 & 0.005 & 0.995 \\
\hline 23 & 3 & 29 & 1 & 1 & 90 & 0.01111111 & 0.98888889 \\
\hline 24 & 3 & 31 & 2 & 1 & 89 & 0.01123596 & 0.98876404 \\
\hline 25 & 3 & 33 & 4 & 2 & 88 & 0.02272727 & 0.97727273 \\
\hline 26 & 3 & 35 & 6 & 2 & 86 & 0.02325581 & 0.97674419 \\
\hline 27 & 3 & 37 & 8 & 2 & 84 & 0.02380952 & 0.97619048 \\
\hline 28 & 3 & 39 & 90 & 82 & 82 & 0.995 & 0.005 \\
\hline 29 & 4 & 32 & 0 & 0 & 90 & 0.005 & 0.995 \\
\hline 30 & 4 & 34 & 1 & 1 & 90 & 0.01111111 & 0.98888889 \\
\hline 31 & 4 & 36 & 3 & 2 & 89 & 0.02247191 & 0.97752809 \\
\hline 32 & 4 & 38 & 12 & 9 & 87 & 0.10344828 & 0.89655172 \\
\hline 33 & 4 & 40 & 90 & 78 & 78 & 0.995 & 0.005 \\
\hline
\end{tabular}



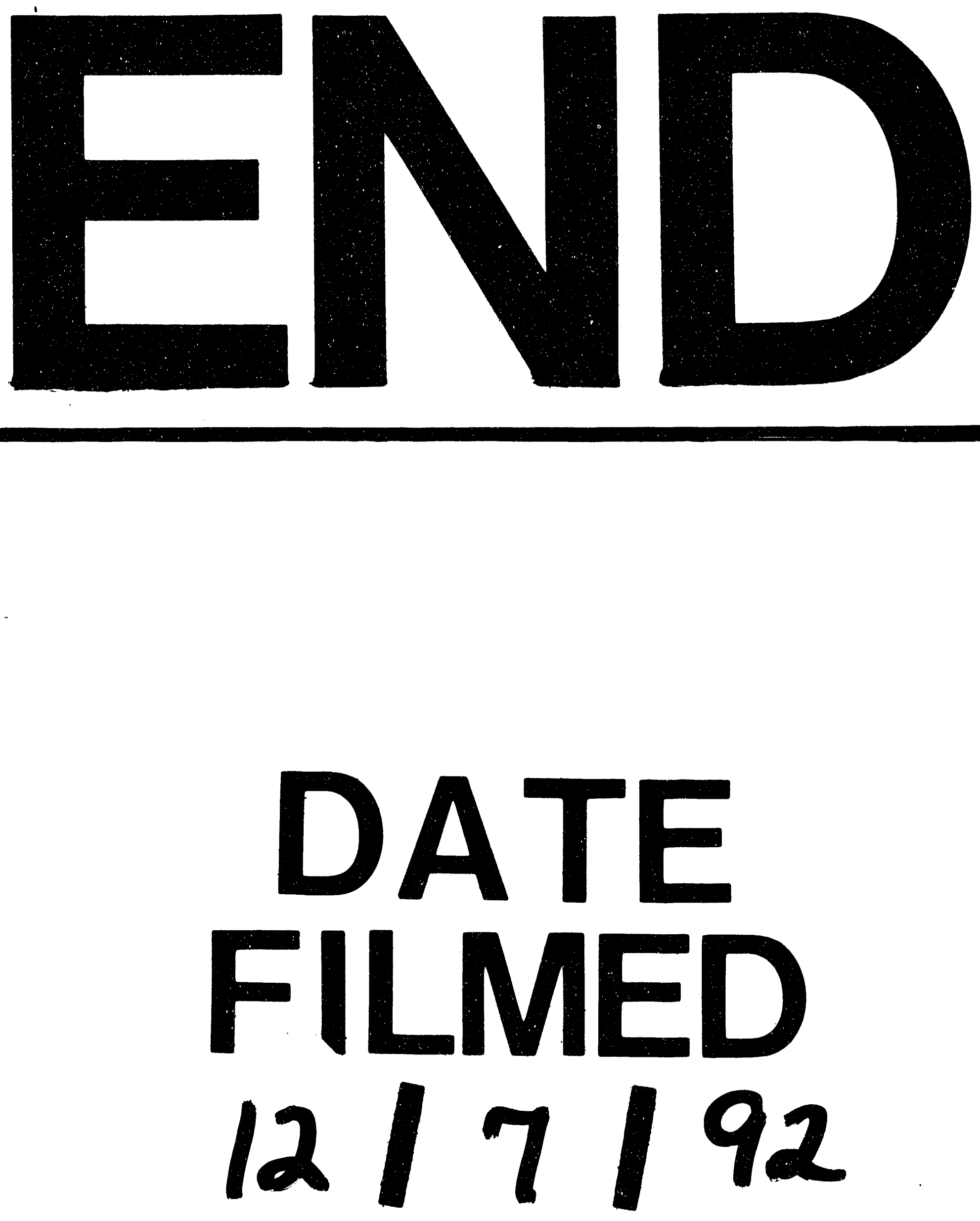
